\title{
Cahier
}

\section{Les pratiques de coordination en situation extrême}

coordonné par Isabelle Bouty, Carole Drucker-Godard, Cécile Godé, Pascal Lièvre, Jean Nizet et François Pichault

Christian Defélix, IAE de Grenoble

JeAn Nizet, Facultés Universitaires de Namur

François PIChault, Université de Liège/ESCP-Europe

Thierry PICQ, EM Lyon 


\title{
Les pratiques de coordination en situation extrême
}

\author{
par Isabelle Bouty ${ }^{233}$, Carole Drucker-Godard ${ }^{234}$, \\ Cécile Godé ${ }^{235}$, Pascal Lièvre ${ }^{236}$, \\ Jean Nizet ${ }^{237}$ et François Pichault ${ }^{238}$
}

Ce cahier spécial est le résultat des travaux de notre groupe de travail qui se réunit régulièrement depuis plus de deux ans et dont l'objectif général est d'approfondir la question de la fabrique de la coordination intra-organisationnelle. Ce phénomène évoque un des problèmes les plus classiquement traités en sciences de gestion. Depuis le début du siècle dernier, les théoriciens s'interrogent sur les différentes façons de coordonner les activités et les hommes, développant tout un ensemble de typologies censées identifier les mécanismes les plus appropriés à mettre en œuvre selon les objectifs poursuivis et les contextes d'action. Ainsi, les théories dominantes de la contingence (Lawrence et Lorsch, 1967 ; Thompson, 1967 ; Van de Ven et al., 1976 ; Mintzberg, 1978 ; Keller, 1994 ; Gupta et al., 1994) suggèrent-elle que les organisations confrontées à une forte incertitude environnementale doivent délaisser les mécanismes formels de coordination au profit de mécanismes beaucoup plus souples. Des contributions plus récentes (Malone et Crowston, 1994 ; Crowston, 1997 ; Gitell, 2000) ont également tendance à adopter une compréhension contingente du phénomène, cherchant à identifier les modes de coordination les plus appropriés à un contexte donné. Pour les tenants de la théorie de la contingence, le choix de la coordination relèverait donc d'une forme d'alignement stratégique au regard des contraintes environnementales (Pichault, 2002).

Cette théorie n'apparaît pas pleinement satisfaisante pour au moins deux raisons. D'une part, elle repose sur une vision essentiellement structurelle de la coordination (Faraj et Xiao, 2006 ; Lechner et Kreutzner, 2010). En effet, les modes de coordination rapportés par la littérature se réfèrent pour la plupart à des arrangements au niveau des dispositifs structurels à mettre en place,

233. IsABelLe Bouty, Maître de Conférences, Ceros, Université Paris Ouest Nanterre la Défense, isabelle.bouty@u-paris10.fr 234. Carole Drucker-Godard, Maître de Conférences, Ceros, Université Paris Ouest Nanterre la Défense, carole.godard@u-paris10.fr

235. CÉCILE Godé, Chercheur au Centre de Recherche de l'Armée de l'Air (CReA), GREDEG UMR 6227 CNRS, Université de Nice Sophia-Antipolis, cecile.gode@inet.air.defense.gouv.fr

236. PAscal Lièvre, Maître de Conférences HDR, Clermont Université \& Groupe ESC Clermont, CRCGM EA 3849 ,

pascallievre@orange.fr

237. Jean Nizet, Professeur extraordinaire aux Facultés Universitaires Notre Dame de la Paix à Namur, Université Catholique de Louvain, jean.nizet@fundp.ac.be

238. Françols Pichault, Professeur à HEC - Ecole de Gestion de l'Université de Liège, Professeur affilié à l'ESCP - EAP Paris, LENTIC, f.pichault@ulg.ac.be 
correspondant par exemple à une démarche de planification des activités (système mécaniste) dans un contexte peu évolutif, ou à la constitution de groupes transversaux (structure organique) visant à favoriser les échanges latéraux dans un contexte plus instable. Dans ces circonstances, la primeur est donnée aux mécanismes simples à décrire, et donc essentiellement formels. Ceci donne la fausse impression que ces mécanismes sont les plus importants, alors que rien ne permet de l'affirmer. Dans ces circonstances, la coordination est abordée à un niveau trop agrégé pour appréhender le phénomène dans toute sa complexité et la partie immergée de l'iceberg demeure inconnue. D'autre part, le niveau de granularité retenu par les théories de la contingence se situe à l'échelon de l'organisation ou de l'unité de travail. II ne permet pas d'analyser la fabrique de la coordination située au niveau des interactions individuelles et d'ouvrir « ces boites noires relativement fermées » (Alsène et Pichault, 2007, p. 65) que représentent aujourd'hui encore les processus de coordination.

Un changement de niveau d'analyse favorise la prise en compte d'autres facteurs de compréhension de la coordination, comme le registre des pratiques. Qu'entendons-nous par pratiques ? II s'agit de ce que font les acteurs en situation et comment ils le font. Les pratiques constituent des connaissances en actes (Piaget, 1974) ou encore des dispositions à agir non conscientisées (Bourdieu, 1977), qui prennent forme en situation toujours singulière. Cette perspective pose fondamentalement la question de l'investigation méthodologique d'un tel objet. Elle interroge également le statut d'une situation d'interaction entre acteurs et porte l'analyse à un niveau collectif, qui ajoute au défi méthodologique. Cet accent mis sur les actions individuelles et collectives nous conduit à considérer la coordination comme une co-construction des acteurs en situation dans une approche interactionniste au sens de Weick (1979). Cette perspective se révèle complémentaire à celle de la contingence décrite plus haut. En synthèse, à la fois les structures contraignent les actions individuelles et collectives et ces dernières influencent les structures (Giddens, 1984).

De nombreuses études (Weick, 1990 ; Weick, 1993 ; Weick et Roberts, 1993 ; Bigley et Roberts, 2001 ; Faraj et Xiao, 2006) mettent en lumière les tensions entre logiques structurelles et acteurs lorsque ces derniers évoluent au sein d'environnements extrêmes. Lorsqu'il s'agit de définir le caractère extrême d'un environnement d'action, il est courant de se référer à la notion de volatilité, qui insiste sur les aspects rapides, dynamiques et discontinus (Bourgeois et Eisenhardt, 1989 ; Wirtz et al., 2007) des changements auxquels les individus font face. Aussi intéressante soit-elle, cette notion apparaît cependant insuffisante pour caractériser à elle seule l'environnement extrême. La référence au concept de « situation extrême de gestion » peut alors être introduite. Nous définissons une situation extrême de gestion comme une situation de gestion (Girin, 1990, Journé et Raulet-Crozet, 2008) évolutive, incertaine et risquée (Lièvre, 2005 ; Lièvre et Gautier, 2009). Selon Girin, une situation de gestion se présente « lorsque 


\section{Les pratiques de coordination en situation extrême}

des participants sont réunis et doivent accomplir dans un temps déterminé, une action conduisant à un résultat soumis à un jugement externe » (1990, p. 142). Elle devient extrême lorsqu'elle articule l'évolutivité, l'incertitude et le risque. Une situation est évolutive lorsqu'elle présente des décalages par rapport à un mode de fonctionnement antérieur (Lièvre, 2005). Ces décalages surviennent de manière rapide et dynamique. Le critère d'incertitude suggère qu'une situation peut être plus ou moins attendue - référence faite à sa probabilité d'occurrence - et/ou plus ou moins anticipable - selon le niveau de prévisibilité du moment et des modalités de la situation (Pichault et al., 2010). Enfin, le risque se réfère à la possibilité qu'un évènement non souhaité survienne et cause des dommages plus ou moins importants à l'organisation et ou à ses acteurs (Lièvre, 2005). Ces dommages peuvent être de nature diverses : physiques, symboliques, matériel, etc.

De ce point de vue, les situations extrêmes de gestion constituent un laboratoire naturel (au sens de non expérimental) et privilégié d'étude de la fabrique de la coordination. En effet, elles sont révélatrices de logiques d'acteurs poussées jusqu'au bout donc plus apparentes, facilitant en cela le travail des chercheurs. Par ailleurs, le caractère paroxysmique et, d'une certaine manière, exemplaire des situations extrêmes est révélateur de situations de gestion plus classiques.

Au regard de notre approche de la coordination, l'objectif général de ce cahier spécial est d'approfondir le phénomène de coordination en situation extrême en examinant les pratiques des acteurs. Comment résolvent-ils concrètement les problèmes de coordination auxquels ils sont confrontés dans des environnements évolutifs, incertains et risqués ? Par notre analyse de la coordination en situation extrême, nous contribuerons à affiner l'appréhension de la coordination en général, ce qui nous conduira à proposer quelques pistes managériales.

\section{Présentation des contributions}

Le premier article, intitulé « L'interprétation des standards en situation extrême : le pouvoir fait-il la différence? » est rédigé par Jean Nizet et François Pichault. Les auteurs s'interrogent sur le rôle que jouent les standards dans la coordination du travail en situation extrême. Si la pensée organisationnelle classique nous a habitués à préconiser, dans ce type de situation, l'adoption de pratiques informelles, reposant sur des relations interpersonnelles entre acteurs, diverses recherches empiriques montrent que, même en contexte incertain et complexe, des pratiques formalisées, se référant notamment à des standards, ont toute leur place et leur pertinence. Ces standards sont évidemment d'origine et de nature diverses, ce qui leur donne des rôles différents. Le travail interprétatif qu'ils permettent est très largement dépendant du système de pouvoir dans lequel ils s'inscrivent. A partir de deux études de cas - l'une dans le cadre d'opérations aériennes de l'OTAN en Afghanistan et l'autre au sein d'un service de néonatologie 
universitaire - les auteurs développent la thèse selon laquelle le caractère plus ou moins structurant des standards mobilisés face aux situations extrêmes est lié aux rapports de pouvoir observables dans chaque situation.

L'article de Cécile Godé, intitulé « Construire le sens par le retour d'expérience : le cas de l'Equipe de Voltige de l'Armée de l'air ", s'attache à comprendre la contribution du retour d'expérience à la construction collective de sens dans les équipes évoluant en environnement extrême. A travers cette question de recherche, l'auteure explore un domaine encore peu investi par la littérature sur la coordination, qui a tendance à insister sur les processus de communication, d'apprentissage et de socialisation pour appréhender la construction collective de sens, mais qui néglige encore les effets du retour d'expérience. La recherche repose sur une étude de cas extrême réalisée auprès de membres de l'Equipe de Voltige de l'Armée de l'Air entre juin et septembre 2010. Confrontés à des enjeux de coordination importants en termes d'intégration des connaissances, les membres de l'Equipe de voltige sont conduits à construire et maintenir collectivement un sens aux situations auxquelles ils font face. Cet article examine le rôle prépondérant joué par le retour d'expérience dans ce processus. En encourageant les échanges de savoir-faire sur des modes le plus souvent informels, le retour d'expérience permet d'impliquer les acteurs dans des cycles d'apprentissage aux niveaux individuel et collectif. Ce faisant, il favorise l'acquisition d'un consensus sur les moyens à mettre en œuvre pour accomplir une ou plusieurs actions.

La troisième contribution, intitulée " Emergence de l'agir collectif dans la course à la voile : rythme et coordination ", s'inscrit dans la thématique générale de la coordination des compétences. Plus précisément, la recherche porte sur la coordination en environnement incertain, et dans une situation distribuée où les rôles, clairement définis et répartis, ne sont pas interchangeables. Sur la base du cadre théorique de la pratique en situation distribuée et à partir des travaux antérieurs sur la coordination vue sous l'angle de la cognition, Isabelle Bouty et Carole Drucker-Godard mènent une étude empirique sur un équipage de voilier en situation de régate. Les observations et analyses issues de l'étude mettent en lumière l'émergence de l'agir collectif autour d'un nombre réduit d'acteurs clés, et d'un rythme à deux temps dans la coordination : conduite et opérations.

Enfin, l'article de Pascal Lièvre et Géraldine Rix-Lièvre, intitulé « Pratiques de coordination d'un collectif informel en situation extrême: une étude de cas "ancrée" au Groenland ", rend compte de la façon dont une expédition polaire, sans standard organisationnel partagé pré-existant, s'y prend pour se coordonner. II apparaît que le chef d'expédition surdétermine les modes de coordination tout au long de l'expédition. C'est à partir de son expérience en tant que chef d'expédition que celui-ci a construit un invariant opératoire dans la manière de conduire une expédition qui l'amène à prendre en charge la conception de l'expédition, à 


\section{Les pratiques de coordination en situation extrême}

coordonner d'une manière classique mais relativement autoritaire la phase de préparation, et à tout faire pour construire et maintenir sa légitimité en tant que chef d'expédition dans la phase de réalisation où l'adaptation doit prévaloir à toute planification. Le choix des équipiers et l'attribution d'un objet symbolique mais aussi pragmatique comme le fusil, utilisé pour gérer la sécurité avec les ours, deviennent des enjeux essentiels dans les modalités de construction globale de la coordination.

\section{Bibliographie}

E. Alsène et F. Pichault (2007), « La coordination au sein des organisations : éléments de recadrage conceptuel », Annales des Mines, série Gérer et Comprendre, Vol. 87, p. 61-77.

G. Bigley et K. Roberts (2001), "The incident command system: High-reliability organizing for complex and volatile task environments", Academy of Management Journal, Vol. 44, $\mathrm{N}^{\circ} 6$, p. 1281-1299.

P. Bourdieu (1977), Le sens pratique, Editions de minuit, Paris.

L. Bourgeois et K. Eisenhardt, (1989), "Strategic decision process in high-velocity environments: Four cases in the microcomputer industry ", Management Science, Vol. $34, N^{\circ} 7$, p. 816-835.

K. Crowston (1997), "A coordination theory approach to organizational process design", Organization Science, Vol. 8, №2, p. 157-175.

S. Faraj et Y. Xiao (2006), "Coordination in fast-response organizations", Management Science, Vol. 52, N8, p. 1155-1169.

A. Giddens (1984), The Constitution of Society. Outline of the Theory of Structuration, Polity Press/Basil Blackwell, Cambridge et Oxford.

J. Girin (1990), «Analyse empirique des situations de gestion : éléments de théorie et de méthode ", in A. C. Martinet (éd.), Epistémologie et sciences de gestion, Economica, Paris, p. 141-182.

J. Gittell (2002), "Coordinating mechanisms in care providing groups: Relational coordination as a mediator and input uncertainty as a moderator of performance effects", Management Science, Vol. 48, №11, p. 1408-1426.

P. Gupta, M. Dirsmith et T. Fogarty (1994), "Coordination and control in a government agency: Contingency and institutional theory perspectives on GAO audits", Administrative Science Quarterly, Vol. 39, №2, p. 264-284.

B. Journé et N. Raulet-Croset (2008), « Le concept de situation : contribution à l'analyse de l'activité managériale dans un contexte d'ambiguïté et d'incertitude », M@n@gement, Vol. 11, No1, p. $27-55$.

R. Keller (1994), "Technology-information processing fit and the performance of R\&D project group: A test of contingency theory", Academy of Management Journal, Vol. 37, No1, p. 167-179.

P. Lawrence et J. Lorsch (1967), Adapter les structures de l'entreprise, Editions d'Organisation.

C. Lechner et M. Kreutzer (2010), "Coordinating growth initiatives in multi-units firms", Long Range Planning, 43, pp. 6-32.

P. Lièvre (2005), Vers une logistique des situations extrêmes, de la logistique de projet du point de vue d'une épistémologie de l'activité d'une expédition polaire, HDR, Université Aix Marseille II.

P. Lièvre et A. Gautier (2009), "Les registres de la logistique des situations extrêmes : des expéditions polaires aux services d'incendie et secours », Management \& Avenir, Vol. 
4, N²4, p. 196-216.

T. Malone et K. Crowston (1994), "The interdisciplinary theory of coordination", ACM Computing Surveys, Vol. 26, N¹, p. 87-119.

H. Mintzberg (1978), The Structuring of Organizations: A Synthesis of the Research, Prentice-Hall Inc.

J. Piaget (1974), La prise de conscience, Presses Universitaires de France, Paris.

F. Pichault (2002), « La question de l'alignement stratégique dans le cadre des nouvelles formes organisationnelles », Revue de Gestion des Ressources Humaines, №46, p. 5975.

F. Pichault, I. Bouty, C. Drucker-Godard, J. Nizet, C. Godé-Sanchez et P. Lièvre (2010), "Coordination practices in extreme situations: Case studies, analytical framework and methodological stakes", $26^{\text {th }}$ EGOS Colloquium, July 2-4, Lisbon, Portugal.

J. Thompson (1967), Organizations in Action: Social Science Bases of Administrative Theory, McGraw-Hill.

A. H. Van de Ven, A. L. Delbecq et R. Koenig (1976), "Determinants of coordination modes within organizations", American Sociological Review, Vol. 41, №2, p. 322-338.

K. Weick (1979), The Social Psychology of Organizing, McGraw Hill Inc.

K. Weick (1990), "The vulnerable system: An analysis of the Tenerife air disaster", Journal of Management, Vol. 16, N³, p. 571-593.

K. Weick (1993), "The collapse of sensemaking in organizations: The Mann Gulch Disaster", Administrative Science Quarterly, Vol. 38, N4, p. 628-652.

K. Weick et K. Roberts (1993), "Collective mind in organizations: Heedful interrelating on flight decks", Administrative Science Quarterly, Vol. 38, p. 357-381.

B. Wirtz, A. Mathieu et O. Schilke (2007), "Strategy in high-velocity environment", Long Range Planning, Vol. 40, p. 293-313. 


\title{
L'interprétation des standards en situation extrême : le pouvoir fait-il la différence?
}

\author{
par Jean Nizet ${ }^{239}$ et François Pichault ${ }^{240}$
}

\section{Résumé}

Cet article s'interroge sur le rôle que jouent les standards dans la coordination du travail en situation extrême. La pensée organisationnelle classique nous a habitués à préconiser, dans ce type de situation, l'adoption de pratiques informelles, reposant sur des relations interpersonnelles entre acteurs. Diverses recherches empiriques montrent cependant que, même en contexte incertain et complexe, des pratiques formalisées, se référant notamment à des standards, ont toute leur place et leur pertinence. Notre contribution s'inscrit dans cette perspective en explorant les standards à l'oeuvre dans les pratiques de coordination en situation extrême. Nous montrons d'abord que ces standards sont d'origine et de nature diverse, ce qui leur donne des rôles différents. Nous précisons ensuite que le travail interprétatif qu'ils permettent est sans doute très largement dépendant du système de pouvoir dans lequel ils s'inscrivent. A partir de deux études de cas - l'une dans le cadre d'opérations aériennes de l'OTAN en Afghanistan et l'autre au sein d'un service de néonatologie universitaire, d'autre part nous développons et discutons la thèse centrale de l'article selon laquelle le caractère plus ou moins structurant des standards mobilisés face aux situations extrêmes est étroitement lié aux rapports de pouvoir observables dans chaque cas particulier.

\section{Abstract}

This paper questions the role of standards in work coordination when facing extreme situations. The classical way of thinking in the organizational theory pleads for the adoption, in such situations, of informal practices based on interpersonal relations between the different actors. Various empirical researches however highlight that, even in uncertain and complex contexts, formalized practices, namely referring to standards, play a crucial role and may be entirely relevant. Our contribution clearly prolongs this perspective by exploring the use of standards in extreme situations as coordination practices. We first clarify that these standards may be of different origin and nature, which gives them different roles. We then consider that the interpretative work they allow probably depends on the power system in which they are inserted. Two case studies - one concerning NATO air force operations in

239. Jean Nizet, Professeur extraordinaire aux Facultés Universitaires Notre Dame de la Paix à Namur, Université Catholique de Louvain, jean.nizet@fundp.ac.be

240. François Pichault, Professeur à HEC - Ecole de Gestion de I'Université de Liège, Professeur affilié à l'ESCP - EAP Paris, LENTIC, f.pichault@ulg.ac.be 
Afghanistan and the other occurring in a university neonatology unit - help us to develop and discuss the central thesis of this paper: the more or less structuring influence of standards in extreme situations is narrowly linked to the power relationships that may be observed in each particular case.

La littérature contingente nous a habitués à établir des liens entre les dispositifs de coordination du travail et différents types de situations. Ainsi, pour March et Simon (1958), une situation stable et prévisible appelle une coordination par plan, à l'opposé d'une situation variable et imprévisible qui requiert plutôt une coordination par rétroaction. Galbraith (1973) estime que les règles et programmes, la voie hiérarchique et les objectifs ne sont des dispositifs valides que lorsque les tâches à exécuter dans les départements interdépendants ne sont pas trop incertaines. Pour les autres cas, l'auteur propose diverses formes de relations latérales interdépartementales, qu'il classe par ordre croissant de capacité à faire face à l'incertitude (les contacts directs entre les gestionnaires, les rôles de liaison, les groupes temporaires, les équipes permanentes, les rôles intégrateurs, les rôles de relation, l'organisation matricielle). Van de Ven et al. (1976) font de leur coté l'hypothèse que, dans une unité de travail, l'utilisation des modes de coordination impersonnel (règles et plans), personnel (canaux de communication verticaux et horizontaux) et de groupe (réunions planifiées et impromptues) varie respectivement en fonction de trois facteurs de contexte: l'incertitude des tâches, l'interdépendance des tâches et la taille de l'unité. Pour Mintzberg (1979) enfin, il existe un continuum dans l'utilisation des mécanismes de coordination, qui renvoie à la complexité du travail à accomplir: «À mesure que le travail d'organisation devient plus difficile, les moyens de coordination employés de façon préférentielle semblent passer [...] de l'ajustement mutuel à la supervision directe, puis à la standardisation des procédés, à celle des résultats, à celle des qualifications et enfin retourner à l'ajustement mutuel dans les situations les plus complexes» (p. 23).

Pendant très longtemps, la pensée organisationnelle n'a donc guère associé les standards à des situations instables, imprévisibles, incertaines et fortement complexes... Elle prédit plutôt, dans ce type de contexte, l'adoption de pratiques essentiellement informelles, reposant sur des relations interpersonnelles entre acteurs.

Cette hypothèse est contredite par diverses recherches empiriques observant que, même en contexte incertain et complexe, des pratiques formalisées, se référant notamment à des standards, ont toute leur place et toute leur pertinence. Ces travaux montrent en outre que la valeur d'un plan, d'une norme ou d'une règle tenait moins dans ce qu'elle est capable de déterminer par elle-même que par le travail interprétatif qu'elle est en mesure de stimuler. Malgré les critiques dont elle a fait récemment l'objet (Basbøll, 2010), on mentionnera à cet égard la célèbre anecdote, rapportée par Weick (1995, p.54), du régiment hongrois perdu 


\section{L'interprétation des standards en situation extrême : le pouvoir fait-il la différence?}

dans les Alpes enneigées, qui finit par retrouver son chemin grâce au sentiment de confiance procuré par l'un d'eux à l'aide d'une carte... des Pyrénées.

Dans un premier temps, nous discutons la manière dont les recherches empiriques existantes nous permettent de documenter la question du rapport aux standards dans les situations extrêmes, en faisant apparaître diverses modalités de ce travail interprétatif.

Dans un deuxième temps, nous présentons deux cas contrastés de coordination du travail en situation extrême: l'un consacrée aux relations entre forces aériennes et terrestres dans le cadre des opérations militaires en Afghanistan ; l'autre concernant le suivi d'une grossesse à risque dans un service de néonatologie universitaire en Belgique.

Dans un troisième temps, nous exposons la thèse centrale de l'article selon laquelle les différences observées dans les pratiques de coordination effectives sont liées aux enjeux qui marquent les relations de pouvoir entre acteurs, dans chaque situation.

\section{Standards et situations extrêmes : un mariage impossible?}

En suivant Ortman (2010), le terme de standard se réfère à des règles qui établissent des exemples, des modèles, des niveaux ou des normes susceptibles d'orienter, voire de déterminer le comportement ou les résultats de ce comportement. II désigne donc un moyen - nécessairement préexistant à la situation considérée - qui spécifie la manière dont la coordination du travail doit s'opérer. Ce moyen peut prendre une forme écrite (règlement, plan de travail, etc.) ou matérielle (chaîne de montage, logiciel, etc.). Dans la mesure où une pratique de coordination s'aligne sur un (ou plusieurs) standard(s), on la qualifiera, très classiquement, de formelle ; dans le cas contraire, on la considérera comme informelle (Blau et Scott, 1962).

Selon les travaux fondateurs de la perspective contingente (Lawrence et Lorsch, 1967 ; Thompson, 1967 ; Mintzberg, 1978), l'évolutivité, l'incertitude et le risque présentés par un contexte particulier ${ }^{241}$ doivent amener l'organisation à abandonner les pratiques formalisées au profit de modes de coordination plus souples et informels, reposant largement sur l'initiative des acteurs. Cette manière de voir est battue en brèche par de nombreux travaux empiriques, montrant que des organisations confrontées aux incertitudes et aux risques adoptent des modes de coordination qui associent des pratiques aussi bien informelles que formelles.

241. Nous nous référons ici à la définition de la situation extrême donnée en introduction de ce cahier. 
L'intéressante étude de Bigley et Roberts (2001) consacrée à la manière dont les services de pompiers réagissent avec une remarquable fiabilité à des situations d'urgence ou de catastrophe majeure, est à cet égard très révélatrice. Pour faire face à ces situations de crise, le fonctionnement fortement bureaucratisé du service qu'ils analysent se combine avec un système de contrôle des incidents (ICS) qui comporte trois principes majeurs :

- des mécanismes de structuration tels que l'élaboration du problème à résoudre, à partir d'un stock de composants organisationnels de base à ajuster en permanence, la ré-allocation des rôles suivant l'évolution des circonstances, un découplage de la structure formelle d'autorité et de dispositifs de prise de décision plus informels vers certains individusclés en fonction de l'expertise requise par la résolution de problèmes particuliers, la possibilité de réinitialiser à tout moment le système d'intervention, etc.;

- le soutien à des formes d'improvisation sous contrainte (étant donné l'existence d'outils, de règles et de routines), en veillant en permanence à éviter les comportements de franc-tireur ;

- des méthodes de gestion cognitive visant à créer un cadre de représentations communes (carte mentale) via d'intenses d'activités de formation et de communication ainsi qu'une organisation systématique de retour d'expérience après chaque incident majeur.

Cette étude nous montre donc que les situations d'urgence n'excluent nullement le recours aux standards, à condition de les enchâsser dans des formes organisationnelles temporaires et flexibles qui en balisent les moments-clés et les modalités, en particulier les écarts tolérés. Ces formes organisationnelles reposent largement sur un cadre commun de représentations, forgé par diverses actions de type cognitif.

C'est à un constat similaire que parviennent Faraj et Xiao (2006). Leur étude de la coordination du travail au sein d'un centre de traumatologie parvient à dégager une alternance de pratiques de coordination fondées sur l'expertise et d'autres de nature dialogique. Les premières reposent notamment sur les processus suivants :

- référence continue aux protocoles et aux " bonnes pratiques », génératrice d'un cadre commun de représentations ;

- structuration de communautés de pratiques à l'intérieur de chaque discipline grâce au coaching exercé par les plus expérimentés ;

- $\quad$ équipes remodelées en permanence en fonction des aléas des arrivées de patients ;

- partage de connaissances, selon plusieurs modalités: débriefing en équipe des différents cas traités sur le mode du face-à-face, colloque animé par l'un des seniors ou encore échange informel à l'occasion des changements d'équipe. 


\section{L'interprétation des standards en situation extrême : le pouvoir fait-il la différence?}

Les secondes prennent à leur tour différentes formes :

- contestation épistémique entre spécialistes ;

- co-construction de sens face à des cas problématiques au-delà des frontières disciplinaires ;

- interventions de spécialistes d'une autre discipline apportant une perspective complémentaire ;

- $\quad$ soutien collectif à la violation exceptionnelle des protocoles dans des cas extrêmes.

A nouveau, c'est la combinaison de pratiques plus ou moins standardisées - basées sur les protocoles et les « bonnes pratiques »- et de pratiques davantage informelles, reposant largement sur l'échange interpersonnel et l'établissement de relations de confiance qui semble garantir la fiabilité des réponses dans des activités à flux tendus.

Dans une étude centrée sur les réponses apportées par le système sanitaire français à la canicule de l'été 2003, Adrot et Garreau (2010) s'intéressent à la façon dont émergent des processus d'improvisation organisationnelle pour faire face à des situations de crise. Ils repèrent à cet égard trois types d'interactions à partir des routines et protocoles existants :

- les interactions discursives, favorisant via de multiples digressions l'émergence de nouvelles pratiques ;

- les interactions de traduction, où une tierce partie parvient à mettre en relation les besoins et attentes de différents groupes d'acteurs, contribuant ainsi à faire émerger un référentiel de règles communes ;

- les interactions fondées sur l'expérience, conduisant à la mise en commun, entre différents professionnels, d'un ensemble restreint de pratiques perçues comme légitimes.

Une fois encore, l'accent est mis sur la nécessité de constituer un cadre commun de représentations, via de multiples échanges interpersonnels, pour " encadrer » les fréquents écarts vis-à-vis des standards, écarts rendus nécessaires par la situation de crise.

Comment s'opère cette combinaison ? Quelle est la nature des standards à l'œuvre dans les organisations confrontées à des situations extrêmes ? Quel(s) rôle(s) jouent ces standards ? Telles sont les questions que nous souhaitons approfondir dans cette contribution. On se montrera notamment attentif aux différences que les standards manifestent, sur les différents points qui suivent.

- Tout d'abord, concernant la nature et l'origine des standards à l'œuvre dans les pratiques de coordination. On a déjà distingué plus haut les standards qui prennent une forme écrite, de ceux qui constituent des dispositifs matériels. On prend également en compte, dans ce texte, les origines différentes des 
standards, en distinguant ceux qui sont mis au point par des acteurs en position d'analyste, qu'ils soient internes ou externes à l'organisation (procédures de travail, normes de qualité, logiciels, etc.) ; ceux qui trouvent leur origine dans les communautés professionnelles auxquelles appartiennent les acteurs concernés (principes éthiques, " bonnes pratiques » étayées dans la littérature scientifique, etc.) et ceux qui renvoient à un cadre réglementaire externe (lois, directives, etc. $)^{242}$. On parlera respectivement, dans la suite du texte, de standards organisationnels, professionnels et institutionnels.

- On s'intéresse également à la mesure dans laquelle les standards influencent effectivement les pratiques de coordination. En effet, des standards peuvent très bien rester en quelque sorte à l'état latent : soit ils n'ont guère d'impact réel sur les pratiques, soit ils influent les pratiques de certains agents, soit encore ils agissent dans certaines circonstances seulement ; à l'inverse, certains standards peuvent s'appliquer en permanence à l'ensemble des agents. Dans le même ordre d'idées, on distinguera les standards qui spécifient de manière précise, stricte, les comportements à adopter, de ceux qui relèvent d'une application plus souple, laissant aux agents une part d'appréciation.

Ce caractère plus moins structurant des standards est largement lié au processus de construction collective de sens dont ils sont souvent à l'origine, et qui les fait évoluer en permanence. Les travaux empiriques que nous avons mentionnés précédemment montrent combien, face aux situations imprévues, les acteurs sont capables de créer des solutions inédites au terme d'un travail interprétatif des situations rencontrées que Weick (1995) qualifie d'enactment. Ils opèrent en réalité une sélection qui consiste en l'application de diverses structures cognitives aux signaux équivoques en provenance de l'environnement, afin d'en améliorer l'intelligibilité. Ces structures sont formées de variables interreliées, construites lors d'expériences antérieures qui, surimposées aux signaux, vont soit rendre les choses encore plus confuses et alors être rejetées, soit en fournir une interprétation raisonnable (Randall \& Procter, 2008). II y aurait donc un processus de renforcement mutuel des interprétations au cours duquel les décisions actuelles sont prises sur base de l'expérience passée et, inversement, où l'expérience passée se voit confirmée par l'action présente. Un tel travail interprétatif est soutenu, comme on l'a vu, par de multiples interactions et échanges d'expériences et débouche sur une construction collective de sens, qui perdure lorsqu'il s'agit de faire face à des situations imprévues. De tels cadres communs de représentation constituent en quelque sorte la base sociale - Rico et al. (2008) parlent à ce sujet de " socle commun de connaissance tacite »- qui balise les réactions des acteurs, lorsqu'ils prennent la liberté de s'écarter des standards.

242. On pourra dégager ici une certaine analogie entre notre problématique des standards à l'œuvre dans les organisations et les travaux de DiMaggio et Powell (1983) sur les pressions isomorphiques entre organisations: nos standards professionnels renvoient sans doute aux pressions de type normatif; nos standards réglementaires ne sont pas sans lien avec les pressions de type coercitif; par contre, nos standards organisationnels ont des liens plus ténus avec les pressions de type mimétique. 


\section{L'interprétation des standards en situation extrême : le pouvoir fait-il la différence?}

Cela signifie-t-il qu'à ce moment, les standards ne jouent plus aucun rôle ? Plusieurs auteurs (Yakura, 2002 ; Sapsed \& Salter, 2004) avancent l'idée selon laquelle des standards techniques comme les représentations graphiques en gestion de projet jouent le rôle d'objets-frontières, dans la mesure où ils offrent aux différentes parties prenantes un référentiel commun leur permettant de mener à bien les négociations et les aménagements temporels nécessaires dans un contexte marqué par la multi-temporalité et les incertitudes. Oswick \& Robertson (2009) vont jusqu'à considérer ces représentations graphiques comme des textes structurant la conversation entre acteurs et leur permettant d'assurer, sur une base formalisée, une certaine maîtrise sur les délais et les tâches à accomplir. Dans une telle perspective, les standards vaudraient moins par leur validité intrinsèque que par le support matériel qu'ils fournissent aux négociations entre acteurs. Par conséquent, ils peuvent être vus comme des " investissements de forme » (Thévenot, 1986), qui aident à réduire la complexité du réel, en en rendant les composantes plus homogènes et plus faciles à maîtriser. Ils auraient dès lors la faculté de créer un langage commun entre les différents protagonistes et les conduiraient désormais à envisager les actions à mener à partir de représentations similaires.

A l'aide des études de cas qui vont être présentées ci-dessous, nous cherchons à rendre compte des différences observées dans la relation complexe entre situations extrêmes, standards et pratiques effectives de coordination. A ce stade, notre hypothèse exploratoire est que la nature et le rôle des standards dans les pratiques de coordination en situation extrême peuvent s'expliquer par les rapports de pouvoir qui ont cours entre les différents acteurs concernés (direction, analystes, ligne hiérarchique, opérateurs, etc.).

\section{Deux cas de coordination en situation extrême}

Pour tester notre hypothèse de recherche, nous procédons à l'analyse de deux cas de coordination du travail en situation extrême. Ces cas sont choisis de manière à faire apparaître des contrastes quant à la nature des standards et quant au rôle qu'ils jouent dans les pratiques de coordination. Le premier se réfère à la coordination entre pilotes de chasse et forces terrestres françaises dans le cadre des opérations aériennes de I'OTAN en Afghanistan. Le second concerne le suivi d'une grossesse à risque au sein du service de néonatologie d'un hôpital universitaire belge.

\section{La coordination air/sol dans les opérations militaires en Afghanistan}

Le premier terrain d'analyse est emprunté aux travaux de Godé-Sanchez (2010a; $2010 b$ ). La chercheuse travaille plus particulièrement sur la coordination entre les équipages d'avion de chasse français et les forces spéciales au sol dont elles soutiennent les opérations. Ce cas s'appuie sur des entretiens individuels 
et collectifs menés auprès de membres d'équipage (pilotes et navigateurs) et auprès des forces spéciales au sol, la participation - en tant qu'observatrice - à des séminaires regroupant ces professionnels, ainsi que la lecture de documents écrits ou audiovisuels (revues professionnelles, enregistrements de dialogues d'équipages, etc. $)^{243}$.

Même si les forces de l'OTAN sont supérieures aux forces talibanes, du point de vue de leur armement, de leurs technologies, etc., elles sont confrontées à des problèmes du fait notamment des caractéristiques du terrain, de la présence de combattants parmi les populations civiles et de la dispersion géographique de leurs unités. Elles rencontrent aussi des difficultés pour récolter des informations fiables. Ces différents éléments, combinés avec les actions menées par l'ennemi, rendent l'environnement de ces forces armées hautement incertain et risqué.

Ceci n'empêche pas la plupart des missions de se dérouler exactement suivant le plan qui a été prévu, à partir des informations préalablement collectées. Avant le départ, la mission est soigneusement préparée en envisageant les différentes éventualités qui peuvent se présenter en termes mécaniques et météorologiques - ce que les membres d'équipage appellent les « what ifs ». A bord, la répartition du travail est stricte entre navigateur et pilote. "Généralement, le navigateur se voit confier la gestion des moyen et long termes (surveillance des menaces électroniques, réglage des fréquences radio, préparation et guidage des armements) tandis que le pilote se concentre sur le pilotage à proprement parler et le(s) tir(s), c'est-à-dire sur le court terme » (Godé-Sanchez, 2010a : 16). Chacune des tâches est dictée par des manuels de vol et des listes de procédures. Les membres de l'équipage communiquent généralement au moyen de mots codes publiés dans la documentation de l'OTAN. La conformité aux procédures est renforcée par la présence de multiples «boutons » dans le cockpit: face à une telle complexité technologique, les boutons favorisent en effet l'acquisition d'automatismes comportementaux et diminuent la charge mentale du pilote (Morel, 2005).

Les contacts avec les contrôleurs au sol suivent également des procédures précises. A une heure déterminée, le pilote établit le contact, fournit au contrôleur un code permettant de l'identifier. Le contrôleur donne à l'équipage des instructions, en se conformant également à une liste standardisée: localisation de l'objectif, description en temps réel des lieux permettant à l'équipage d'identifier la cible, etc. Le contrôleur au sol donne alors au pilote l'ordre de tirer ou non. Notons que s'ils appartiennent aux forces françaises, l'un et l'autre utilisent le même outil de géolocalisation qui leur permet de partager à tout instant, de manière automatique, la même image de la situation tactique. Cet outil participe indiscutablement à la constitution d'un cadre commun de représentations.

243. Outre les informations que nous avons extraites des travaux de l'auteure, celle-ci nous a fourni, lors d'un entretien, quelques données complémentaires qui nous étaient utiles pour tester les hypothèses spécifiques de cette contribution. 


\section{L'interprétation des standards en situation extrême : le pouvoir fait-il la différence?}

Toutes ces procédures formelles ont été apprises par les membres d'équipage et par les forces au sol au cours d'un parcours de qualification comprenant des cours théoriques, des simulations de vol, des vols réels, etc. En ce qui concerne les équipages, ce parcours est jalonné par l'acquisition de compétences techniques et comportementales précises qui permettent de passer au statut de pilote opérationnel, de sous-chef, puis de chef de patrouille, de chef de mission, etc. Une progression similaire existe dans le cas des forces spéciales au sol. Pour devenir contrôleur aérien, il s'agit également de suivre une formation complémentaire. Au cours de ces diverses formations, les apprenants sont en outre amenés à côtoyer les équipages de retour d'Afghanistan, ce qui les aide à nouer avec eux des liens interpersonnels.

II peut arriver que les forces engagées soient confrontées à des situations tactiques imprévues qui conduisent l'équipage à l'écarter du plan initialement prévu. Par exemple, les forces au sol se trouvent à proximité des forces ennemies: l'équipage peut alors décider de ne pas ouvrir le feu, mais de changer de registre en adoptant une autre solution type (telle que le vol à basse altitude en vue d'effrayer et de déstabiliser l'ennemi). Dans ce type de circonstances, la division des tâches et les règles qui prévalent généralement peuvent être redéfinies. Les deux membres de l'équipage sont désormais centrés sur le court terme. II leur arrive alors de décider du mode d'intervention sans se référer désormais aux contrôleurs. A ce moment, ils n'échangent plus entre eux par des mots codes, mais retournent au langage naturel, du fait du stress et aussi, comme le dit un interviewé parce que "l'objectif, c'est de faire passer l'info, quel que soit le mode " (Godé-Sanchez, 2010a, p.21). Remarquons que l'écart dont il est question ici concerne le mode d'échange entre membres de l'équipage pour parvenir au choix de cette solution et non la solution elle-même, qui fait quant à elle partie d'un ensemble de solutions standardisées.

Signalons encore que, dans la réalisation de ces missions, qu'elles soient ou non routinières, les équipages et les forces au sol sont guidés par des règles strictes décidées par les responsables politiques, qui définissent les circonstances des engagements et certaines limites à respecter. Le gouvernement français se montre notamment très sensible au risque de dommages collatéraux liés aux frappes aériennes et dissuade par conséquent de recourir aux tirs dès que la proximité de la cible avec la position des forces amies ou avec des zones civiles sensibles (école, mosquée, etc.) est trop importante (Lert, 2009).

De telles règles d'engagement correspondent à ce que nous avons appelé des standards institutionnels. Pour le reste, c'est surtout la présence massive de standards organisationnels qui frappe dans ce cas. Certains d'entre eux reposent sur une base matérielle (les boutons dans le cockpit), tandis que les autres prennent une forme écrite (manuels de navigation, procédures pour les échanges avec les contrôleurs, listes de mots codes, etc.). Lorsqu'on a affaire 
à des missions routinières, ces standards sont d'application et ils commandent la plupart des comportements des personnes, y compris leurs communications. Dans le cas de missions non routinières, plusieurs d'entre eux ne sont plus d'application et sont donc largement transgressés.

Lors des entretiens, les pilotes et navigateurs insistent sur l'importance des automatismes liés à ces standards, d'ailleurs renforcés du fait qu'un équipage est souvent constitué de manière stable dans le temps. Ils permettent aux opérateurs d'acquérir une vision commune de leur mission. Ils leur permettent surtout, lorsqu'ils font face à une situation imprévue, de libérer du temps et de l'attention pour les tâches urgentes, devenues prioritaires. "Dans ces circonstances, ils doivent s'adapter en temps réel et le fait qu'ils aient pris l'habitude de travailler ensemble en détachement leur permet de gagner un temps précieux pour interpréter la situation, lui redonner du sens et explorer des solutions tactiques » (Godé-Sanchez, 2010a, p.25). Le débriefing informel réalisé le soir au bar de l'escadron acquiert ici toute son importance : les équipages échangent sur les événements de la journée, leur propre ressenti émotionnel, les solutions qu'ils ont mises en place ou les erreurs qu'ils ont commises. « Le bar de l'escadron est ainsi un endroit où les membres d'équipages construisent progressivement un stock de connaissances communes qui leur donnent une connaissance partagée adéquate de leur travail et des rôles qu'ils occupent dans l'équipe » (GodéSanchez, 2010b, p.76). On retrouve ici la construction collective de sens, fondée sur l'apprentissage et les expériences communes et facilitée par la stabilité des équipages. Un interviewé compare la situation d'un équipage à celle d'une équipe de football qui, au fur et à mesure des entraînements, acquiert une même vision du jeu.

En outre, pour chaque mission, s'ensuit un débriefing plus formel, dans une salle ad hoc, souvent en présence du chef de mission et/ou du chef de patrouille. Un tel débriefing suit cette fois un canevas très précis qui évalue le respect des standards, la légitimité du travail interprétatif effectué et l'éventuelle nécessité d'apporter un complément aux standards existants. Si le vol a connu un problème, il est possible de faire intervenir les différentes parties prenantes concernées (contrôleurs, mécaniciens, etc.) et, si le problème est d'une certaine ampleur, il donne lieu à la rédaction d'un document, transmis à la hiérarchie. Celui-ci peut remonter jusqu'à l'état-major qui décide alors des aménagements à apporter aux standards en vigueur. Ainsi les règles d'engagement très strictes édictées par les autorités françaises au début du conflit ont dû être assouplies face aux nombreux retours d'expériences qui faisaient état de la difficulté d'apporter un soutien aux forces alliées américaines, dont les règles d'engagement s'avéraient beaucoup plus lâches (Lert, 2009).

La confiance mutuelle se révèle également cruciale. Elle permet de transformer partiellement le stress que suscitent les situations imprévues en émotions 


\section{L'interprétation des standards en situation extrême : le pouvoir fait-il la différence?}

davantage positives et de se concentrer sur les tâches urgentes à réaliser. "La confiance, c'est une garantie de protection mutuelle », explique un pilote interviewé (Godé-Sanchez, 2010a, p.22). Les rencontres professionnelles de la vie en escadron, certaines activités dites « de cohésion » comme des rencontres sportives, ainsi que les relations amicales qui s'y développent nourrissent cette confiance entre les personnes. Pour un navigateur, « l'escadron, c'est une tribu. Faire confiance, c'est devenu culturel » (Godé-Sanchez, 2010a, p.22).

\section{Le suivi d'une grossesse à risque dans un service de néonatologie}

Notre second terrain d'analyse est un service de néonatologie d'un centre hospitalier universitaire belge. Le cas précis que nous analysons ici met en scène la manière dont les médecins, infirmiers, ainsi que des acteurs extérieurs à l'hôpital, se concertent par rapport à une patiente qui connaît une grossesse problématique. Ce cas est constitué, au départ, d'un document audiovisuel d'une douzaine de minutes réalisé par une chaîne de télévision publique belge. Nous avons pu compléter ce matériel audiovisuel par des entretiens approfondis avec les deux médecins qui avaient en charge la patiente et par plusieurs dizaines d'entretiens de contexte réalisés auprès de responsables administratifs, médicaux et infirmiers, dans le cadre d'une recherche-intervention consacrée, par l'un des auteurs, à la professionnalisation de la fonction ressources humaines au sein de cet hôpital universitaire.

Une des spécificités du cas est de s'inscrire dans la durée. La patiente est en effet suivie sur une période de plusieurs semaines. Nous mettons ici l'accent sur deux moments importants.

Le premier intervient après 24 semaines de grossesse. La patiente, qui a été hospitalisée suite à une rupture partielle de la poche des eaux, vient de subir une échographie. Le docteur S, obstétricien et spécialiste en diagnostic prénatal, a repéré « quelque chose d'anormal, un gros pli », qui pourrait laisser penser à une malformation chromosomique du fœtus. II s'en ouvre à deux collègues présents à ce moment dans le service : " je n'ai aucune étiquette franche, je ne peux rien lui dire de définitif ». Ils conviennent de laisser évoluer la situation, sans en informer la patiente et sans proposer d'autres examens, de manière à « être le moins agressif possible ».

Le deuxième moment intervient deux semaines plus tard : le cas est débattu lors du " colloque " qui se tient tous les lundis matin dans le service, cette fois en l'absence du docteur S, retenu par d'autres activités. La réunion regroupe une douzaine de personnes : des infirmiers et médecins (gynécologue, anesthésiste, chirurgien pédiatre, etc.) travaillant dans le service, ainsi que des médecins stagiaires. Elle est animée par le docteur $F$, chef du service. Les clichés échographiques dont on dispose à ce moment semblent montrer que l'enfant est un petit garçon, ce qui réduit les risques de malformation. Le docteur $\mathrm{F}$ fait état 
des recherches médicales qui montrent que, si on provoque l'accouchement à ce stade, les chances qu'a l'enfant de vivre et d'être normal sont faibles : " elles se situent aux environs de $20 \%$ ». Un médecin explique qu'il a donné hier à la patiente des informations sur son état, car elle se montrait inquiète. Le docteur $F$ approuve : " cela fait partie de la politique de la maison de donner l'information au fur et à mesure que se manifestent les inquiétudes et les sollicitations des patientes ». A la question du docteur $F$, « la maman est-elle très investie dans sa grossesse ? », une autre collègue précise: " elle est prête à tout ... pour un enfant qui n'est pas mal formé ». Le colloque se conclut sur l'option de réaliser un examen supplémentaire - une prise de sang - qui donnera des informations plus précises sur l'existence ou non de la malformation. Quelques heures après la réunion, le docteur $\mathrm{F}$ communique l'option qui s'est dégagée au docteur S. Celui-ci interrompt rapidement son interlocuteur en lui disant qu'il est lui-même arrivé aux mêmes conclusions et qu'il pratiquera le prélèvement le lendemain. Le docteur F clôt l'entretien en souhaitant à son collègue bonne chance.

Au cours de nos interviews avec les docteurs $F$ et $S$, nous apprenons qu'un certain nombre de patientes, parfois sous l'impulsion de leur médecin traitant ou de leur gynécologue, introduisent des actions en justice contre les médecins du service de néonatologie pour des motifs très divers, liés aux aléas de la grossesse. Forts de leurs expériences passées, les docteurs $F$ et $S$ ont pris l'habitude, dans ce cas comme dans les autres suivis de grossesse, de documenter, par écrit, toutes leurs décisions.

Les descriptions qui précèdent nous montrent que, dans ce cas, les acteurs sont confrontés à deux types d'incertitudes : d'abord - et essentiellement - celle qui a trait à l'état de la patiente et de son enfant ; ensuite, les actions en justice susceptibles d'être entreprises par les patientes et/ou par leur médecin traitant, en cas d'erreur médicale.

Dans quelle mesure les pratiques de coordination mises en place pour faire face à ces incertitudes révèlent-elles l'existence de standards?

- Dans leurs décisions d'intervention, les acteurs se réfèrent aux recherches médicales qui leur fournissent ce qu'ils appellent des guidelines (dans le cas présent, il s'agit de l'estimation de survie de l'enfant, en cas d'accouchement prématuré). II s'agit ici clairement de standards professionnels.

- Lors du " colloque », le docteur F fait également référence à la politique spécifique de l'institution hospitalière en matière d'information de ses patients. II s'agit ici de standards de type organisationnel, par lesquels certaines valeurs, certains principes, certaines orientations philosophiques sont diffusés et, au besoin, rappelés, aux membres de l'organisation. 


\section{L'interprétation des standards en situation extrême : le pouvoir fait-il la différence?}

Ces deux types de standards sont censés fournir une ligne de conduite générale chaque fois qu'une situation similaire se présente : ils contribuent donc à éviter les réponses totalement ad hoc. Ils sont toutefois très loin de commander les multiples pratiques de coordination concrètement employées. Ils sont présents, certes, mais de manière relativement limitée. En effet, le rappel de la " politique de la clinique » intervient ex post, et ne concerne qu'un aspect somme toute assez mineur du travail de l'équipe : le moment où l'on livre à la patiente les informations sur son état de santé. Quant aux guidelines fournis par la recherche, nos interviews montrent qu'elles doivent en permanence être appréciées en fonction des caractéristiques spécifiques de la patiente que l'on a en face de soi : son état médical spécifique, mais aussi la manière dont elle vit sa grossesse, la manière dont elle envisage la naissance éventuelle d'un enfant handicapé, etc. Or ce travail d'appréciation s'opère par des échanges entre médecins, entre médecins et infirmiers, etc.

Comment les acteurs font-ils face à la seconde incertitude ? En consignant systématiquement par écrit, dans des dossiers, les différents actes médicaux qu'ils ont posés. On retrouve ici une pratique formalisée, certes, mais qui ne se réfère pas à des standards élaborés par de quelconques analystes. Ce sont à nouveau des échanges entre professionnels - en particulier les médecins - qui sont à l'œuvre ici. L'accumulation de ces écrits peut néanmoins contribuer, à son tour, à l'élaboration de nouveaux standards professionnels par le biais de publications dans des revues scientifiques spécialisées.

\section{Discussion : rôles des standards et jeux de pouvoir}

Ces deux situations sont, à l'évidence, contrastées du point de vue du type de standards à l'œuvre et de leurs rôles respectifs.

Dans le cas des forces militaires en action en Afghanistan, nous avons affaire à des standards d'origines différentes : des standards institutionnels émanant des autorités politiques, qui se spécifient et se combinent avec d'autres standards au niveau organisationnel et qui, par le biais de formations, deviennent une des composantes des standards professionnels. Ces divers standards qui s'emboîtent et se renforcent sont d'application dans les missions de routine, c'est-à-dire dans la grande majorité des cas. Ils spécifient alors de manière très précise, très stricte, les comportements à adopter - jusqu'aux mots à utiliser dans les communications entre les membres de l'équipage et entre ceux-ci et les inspecteurs au sol. On peut dire que, dans ces circonstances, les standards structurent de manière maximale la coordination entre les agents. A l'inverse, lorsque les équipages sont confrontés à des imprévus, l'application des standards est momentanément suspendue ; elle laisse la place à des mécanismes de coordination plus informels, à savoir des échanges non structurés, en langage naturel, entre pilote et navigateur. Ceci 
pourrait laisser penser qu'on se trouve bel et bien dans un cas d'application des hypothèses mécanistes de la théorie contingente: dans une situation de routine, les standards sont d'application; dans une situation exceptionnelle, ce sont des relations interpersonnelles qui l'emportent. Les observations en provenance du terrain sont cependant plus complexes et plus subtiles : le fait que les standards aient été appris au cours de formations, qu'ils soient appliqués en permanence, qu'ils fassent l'objet d'une évaluation de leur efficacité au retour des missions, tout cela participe de l'élaboration d'un cadre commun de représentations. En outre, la stabilité des équipages pendant la durée du détachement en Aghanistan forme une base sociale solide, faite de confiance mutuelle, sur laquelle des "improvisations » légitimes peuvent se construire dans les situations non routinières : elle permet en effet de mieux gérer le stress et de prendre des décisions adéquates.

Dans le cas du service de néonatologie, les standards s'avèrent beaucoup moins structurants des pratiques de coordination effectives. Les quelques tentatives de rappel des standards organisationnels (la " politique de la maison ») ont une portée assez limitée, comme on l'a vu. Quant aux standards professionnels, ils restent la plupart du temps à l'état latent. Quand ils sont mobilisés, ils font partie d'un travail d'enactment qui laisse une marge d'appréciation assez large aux médecins (on notera notamment le recours aux probabilités statistiques de survie du bébé pour encadrer la décision d'intervention): ceux-ci confrontent le contenu du standard avec d'autres informations, notamment liées à l'état de santé ainsi qu'à la personnalité et aux souhaits des patientes. Bref, les standards, essentiellement d'origine professionnelle, paraissent structurer de manière relativement limitée la coordination entre les acteurs.

A plusieurs moments de l'évolution du cas, on peut observer le docteur $\mathrm{S}$ en train de confronter les guidelines issues de ses standards professionnels à l'expérience concrète qu'il a de la situation et à celle vécue par ses collègues. Le colloque est le lieu institué pour partager ces expériences et constituer progressivement un cadre commun de représentations ${ }^{244}$. Une fois encore, on voit ainsi émerger un processus collectif de construction du sens. Toutefois, au terme du processus de décision, c'est le gynécologue en charge de la patiente qui, nourri de ces diverses informations (les guidelines, le consensus résultant du colloque, sa propre expérience de la situation), se retrouve seul responsable du risque à prendre (le prélèvement sanguin peut en effet accélérer le risque de mort prématurée du bébé). Les standards professionnels ne constituent donc pour lui qu'un signal parmi d'autres susceptibles de faire l'objet d'un enactment. Toutefois, ils représentent à ses yeux une source d'information d'autant plus importante qu'elle lui permet de faire face au second type d'incertitude : les

\footnotetext{
244. L'interview des deux médecins concernés nous a fait apparaître d'autres dispositifs contribuant à forger ce cadre de références commun : les séances de formation avec les stagiaires, les groupes de parole des infirmières, etc. Ces autres dispositifs ne seront guère approfondis ici, afin de ne pas allonger inutilement le propos.
} 


\section{L'interprétation des standards en situation extrême : le pouvoir fait-il la différence?}

actions en justice susceptibles d'être entreprises en cas d'erreur médicale. Une fois encore, ce n'est pas l'application mécanique du standard qui est observée, mais sa mobilisation parmi d'autres sources d'information, dans le cadre du travail de documentation et de justification ex post des actes médicaux posés.

L'analyse des cas montre donc que, lorsqu'on a affaire à des standards institutionnels ou organisationnels, le travail interprétatif - ou encore l'émergence de pratiques d'improvisation - n'apparaît qu'aux seuls moments où les standards ne s'appliquent plus; il comporte donc un caractère exceptionnel. Inversement, lorsque les standards sont principalement d'origine professionnelle, comme dans le second cas, le travail interprétatif s'effectue de façon continue, par la confrontation systématique de différentes sources d'information et l'échange interpersonnel fréquent avec les pairs.

Si on tente de généraliser ce résultat, une première relation semble s'esquisser entre l'origine des standards et leur caractère plus ou moins structurant en matière de coordination. Aux standards institutionnels et organisationnels serait lié un travail d'interprétation de faible ampleur; aux standards professionnels, un travail interprétatif plus important.

Figure 1 : Origine des standards et modalités du travail interprétatif

Standards institutionnels/organisationnels $\leftrightarrow$ travail interprétatif faible

Standards professionnels $\leftrightarrow$ travail interprétatif fort

Raffinons cependant le raisonnement. Les standards n'agissent pas en tant que tels : le travail interprétatif dont ils font l'objet est largement dépendant des relations de pouvoir entre acteurs impliqués dans la situation.

Dans le cas des opérations aériennes en Afghanistan, on se trouve dans une structure militaire fortement bureaucratisée. La capacité de structuration des standards institutionnels et organisationnels y est associée à l'influence prédominante de la ligne hiérarchique et des analystes. Dans un tel contexte, les équipages sont supposés appliquer strictement les standards - rappelons-nous le rôle structurant des supports matériels tels que les boutons dans le cockpit, favorisant l'acquisition d'automatismes comportementaux au-delà de la simple présence de règles écrites - mais ils reçoivent l'autorisation de s'en écarter en cas de situation extrême (par exemple, le constat in situ que les forces au sol se trouvent à proximité immédiate des forces ennemies). S'enclenche alors un processus d'ajustement des standards, qui débute par les improvisations des acteurs de terrain enchâssées dans un cadre commun de représentations, fondé sur la confiance mutuelle entre membres des équipages. Un tel processus se poursuit par le débriefing informel au bar et par le débriefing plus formel de chaque mission qui peut déboucher, le cas échéant, sur une adaptation des 
standards par l'état-major : dans ce cas, les standards gagnent en efficacité et parviennent à rester suffisamment génériques (c'est-à-dire pertinents dans le plus grand nombre de situations possibles). On peut ici évoquer la distinction classique, proposée par Reynaud (1997), entre régulation autonome, régulation de contrôle et régulation conjointe. Le cycle interprétatif que nous avons qualifié d'exceptionnel est en effet étroitement balisé par la régulation conjointe (à dominante contrôle) du système de pouvoir dans lequel se déroulent les opérations aériennes : il ne débouche qu'occasionnellement sur des évolutions des standards eux-mêmes. Et quand celles-ci ont lieu, elles s'effectuent sous la responsabilité des responsables hiérarchiques et des analystes de l'état-major, et non des opérateurs eux-mêmes.

Dans le cas du suivi de grossesses à risque en milieu hospitalier, le système de pouvoir est d'une tout autre nature : les acteurs de terrain les plus qualifiés (chirurgiens, anesthésistes, gynécologues, etc.) y disposent d'une grande capacité d'influence, principalement fondée sur leur pouvoir d'expertise. En outre, dans un hôpital universitaire, ils cumulent les atouts que leur procurent l'expertise médicale et l'expertise scientifique. Dans ce cadre, la capacité de structuration des standards (guidelines) reste très limitée, d'autant plus que la majorité d'entre eux est d'origine professionnelle. Les opérateurs qualifiés sont censés mobiliser plusieurs sources de savoir, et le standard - le plus souvent sous forme écrite - ne constitue que l'une d'entre elles. Ce dernier est donc considéré comme une toile de fond et peut rester largement implicite ou bien être convoqué à des moments spécifiques, en fonction de l'évolution et des enjeux de la situation. Et même lorsqu'il est convoqué, il reste l'objet d'interprétations diverses de la part des spécialistes, qui le soumettent à débat dans le cadre d'échanges latéraux où sont croisées des informations d'origine multiple (en provenance du médecin traitant, de la patiente elle-même, d'autres collègues, etc.). Notons que ce travail interprétatif peut être de type individuel quand le gynécologue prend seul la responsabilité d'une intervention risquée, une fois qu'il estime disposer de suffisamment d'informations et de conseils. Mais il peut être aussi de type collectif si l'on prend en compte l'échange d'informations qui s'effectue au cours du colloque animé par un médecin senior, qui n'est pas sans rappeler la structuration de communautés de pratiques via le coaching d'un praticien expérimenté observée par Faraj et Xiao (2006) dans leur analyse du fonctionnement d'un service de traumatologie.

Ilestcependantfrappant de constaterque le docteur Stientàgarderla responsabilité individuelle du travail interprétatif, en interrompant son interlocuteur, le docteur $F$, lorsque celui-ci lui rapporte les orientations collectives issues du colloque, pour l'informer qu'il était déjà arrivé par lui-même aux mêmes conclusions. En termes plus politiques, on le voit ici revendiquer la maîtrise de la zone d'incertitude que représente la décision d'intervenir ou non. 


\section{L'interprétation des standards en situation extrême : le pouvoir fait-il la différence?}

Le travail interprétatif est donc bel et bien continu et conduit à l'élaboration de solutions chaque fois spécifiques, en fonction des caractéristiques précises de la situation extrême et de son évolution. La multiplication de ces solutions spécifiques émanant des spécialistes finit par donner lieu à une adaptation des guidelines par l'intermédiaire des publications scientifiques.

En outre, la mobilisation des standards poursuit ici parallèlement un autre objectif. II s'agit en effet de faire face au second type d'incertitude mentionné plus haut: le risque d'actions en justice. Les standards sont alors convoqués, au même titre que d'autres sources d'informations - y compris des éléments factuels -, pour documenter et justifier chacun des actes médicaux posés dans le cadre d'un suivi de grossesse. L'utilisation des standards fait à nouveau partie d'un travail interprétatif : soit, le spécialiste choisit, pour tel acte précis, de faire directement référence aux guidelines en montrant qu'il s'y est conformé; soit, il explicite le fait qu'il a décidé de s'en écarter après concertation avec ses collègues et en fonction des informations dont il disposait à ce moment-là. Ce second cas de figure rappelle le soutien collectif à la violation des protocoles à nouveau observé par Faraj et Xiao (2006).

En synthèse du cas de suivi de grossesses à risque, on relèvera que, pour faire face aux deux types d'incertitude que nous avons distingués, l'interprétation et l'adaptation des standards restent entièrement dans les mains des opérateurs qualifiés, sans guère d'intervention d'autres acteurs. On se trouve donc ici dans une forme de régulation largement autonome.

La manière dont les standards sont mobilisés et « nourrissent " le travail interprétatif est donc très fortement marquée par la distribution des ressources entre acteurs. Dans le cas des opérations aériennes en Afghanistan, c'est-àdire dans un contexte de relations de pouvoir fortement asymétriques, il ne s'enclenche que dans des situations précises, au moment où l'opérateur peut se baser sur des ressources très spécifiques (croisement d'informations disponibles à un moment précis) pour se permettre des écarts par rapport à ce qui était prévu. Cet écart fera immédiatement l'objet d'un débriefing et sera, le cas échéant, transformé en nouvelle règle une fois que la ligne hiérarchique et les analystes de l'état-major auront repris la main sur l'interprétation de la situation extrême. On pourrait évoquer à cet égard le cercle vicieux bureaucratique, mis en évidence par Merton (1960) : l'écart par rapport à la règle donne lieu, in fine, à la production de nouvelles règles. Dans le cas du suivi de grossesses à risque, où les relations de pouvoir sont bien davantage symétriques, le travail interprétatif est permanent et peut être même considéré comme l'apanage des opérateurs qualifiés. Ces derniers disposent de toutes les ressources nécessaires (expertise, informations croisées, etc.) pour s'écarter à tout moment des guidelines, qui ne constituent finalement que de simples balises de leur action. Rappelons à ce sujet que Crozier et Friedberg (1977) font de l'application même des règles 
une zone d'incertitude et, par conséquent, une source de pouvoir. Dans un tel contexte, toute tentative d'intrusion ou de restriction du travail interprétatif individuel risque d'être rapidement mise en échec : rappelons-nous la vigueur avec la laquelle le Docteur S interrompt le Docteur F, au cours de leur face-àface, pour lui rappeler qu'il est arrivé par lui-même aux mêmes conclusions que l'assemblée des collègues.

Notre article souligne la nécessité d'introduire la question du pouvoir dans l'analyse du travail interprétatif des opérateurs en situation extrême. Les écarts que ceux-ci se permettent vis-à-vis des standards ont en effet d'autant plus de chances d'être de forte intensité que les rapports de pouvoir entre acteurs concernés par la situation extrême sont symétriques : dans ce cas, les standards existent essentiellement sous forme écrite et ne constituent que l'une des sources du travail interprétatif. A l'inverse, les écarts seront plus étroitement cadenassés (faible intensité du travail d'interprétation) dans le cas de relations de pouvoir asymétriques: les standards peuvent alors adopter non seulement une forme écrite mais aussi matérielle. On voit ainsi s'esquisser une intéressante relation entre le type de rapports de pouvoir, l'origine et la forme des standards et l'ampleur du travail interprétatif en situation extrême.

Figure 2 : Rapports de pouvoir et modalités du travail interprétatif

Rapports de pouvoir asymétriques et régulation de contrôle dominante $\Leftrightarrow$ Standards institutionnels/ organisationnels, de forme écrite et/ou matérielle $\Leftrightarrow$ travail interprétatif faible

Rapports de pouvoir symétriques et régulation autonome $\Leftrightarrow$ Standards professionnels de forme écrite $\Leftrightarrow$ travail interprétatif fort

\section{Conclusion}

Notre contribution s'inscrit dans la ligne des travaux de recherche qui s'interrogent sur le rôle des standards dans la coordination du travail en situation extrême. De telles recherches, qui procèdent à des analyses empiriques fouillées, montrent que, contrairement à ce qu'affirmaient certains travaux classiques, les standards ont toute leur raison d'être dans ce type de situations, où ils se combinent avec des mécanismes de coordination plus informels. Elles montrent également que, si les standards peuvent certes influencer directement les pratiques, leurs effets tiennent aussi, pour une grande part, au travail interprétatif auquel ils donnent lieu. Notre contribution, qui s'appuie sur deux cas contrastés d'organisations confrontées à des situations extrêmes - des forces armées engagées dans des frappes aériennes en Afghanistan et le service de néonatologie d'un hôpital universitaire confronté à une grossesse à risques - complète et affine ces résultats, de deux manières. Elle suggère d'abord que les standards peuvent, suivant les situations, présenter diverses origines - professionnelle, organisationnelle et institutionnelle - et que, en fonction de celle-ci, l'ampleur du travail interprétatif 


\section{L'interprétation des standards en situation extrême : le pouvoir fait-il la différence?}

peut varier: ce travail sera plus intense dans le cas des standards professionnels, plus faible dans le cas des standards organisationnels ou institutionnels. Elle suggère ensuite que le premier cas de figure correspond à des rapports de pouvoir plus asymétriques, allant de pair avec une prédominance de la régulation de contrôle, tandis que le second renvoie à des rapports plus symétriques, liés à la prédominance de la régulation autonome.

Notre contribution comporte bien évidemment des limites. Une des plus importantes réside dans la toute petite taille de notre échantillon. II conviendrait d'élargir cette base empirique pour valider (le cas échéant, invalider) les conclusions auxquelles nous aboutissons et pour les affiner. Ainsi, une des variables de notre modèle oppose un travail interprétatif intense versus faible. II conviendrait de mieux préciser ce que recouvrent ces deux termes: en particulier, le travail d'intensité faible prend-il toujours concrètement la forme d'un travail discontinu, comme dans le premier cas examiné? Ou bien ce travail se concentre-t-il sur certaines catégories de pratiques, à l'exclusion d'autres? Autre point qui mériterait d'être approfondi: dans l'éventualité de rapports de pouvoir asymétriques, nous avons montré que le travail interprétatif des opérateurs peut être mené tantôt collectivement, tantôt individuellement. Ceci n'est pas sans rappeler les conclusions auxquelles nous aboutissions dans d'autres travaux, relatifs cette fois aux pratiques de gestion des ressources humaines (le temps de travail, les promotions, les formations, l'engagement du personnel, etc.). Nous montrions que, dans des contextes très professionnalisés, ces pratiques peuvent faire l'objet de règles élaborées collectivement entre opérateurs (ce peut être le cas, par exemple pour les promotions), mais qu'elles peuvent aussi rester de l'ordre de la maîtrise individuelle (par exemple, pour les formations) (Pichault et Nizet, 2000, p. 136-145). En matière de coordination, d'autres analyses seraient nécessaires pour déterminer dans quelles circonstances le travail interprétatif collectif prime sur le travail individuel, et vice versa; également pour explorer les tensions, les conflits éventuels liés à la coexistence de ces deux modalités, voire les diverses catégories d'acteurs susceptibles de les prendre en charge...

Le modèle auquel nous aboutissons revient donc à penser la coordination en situations extrêmes en articulant trois grandes catégories de variables: d'abord l'origine et la nature des standards, ensuite les modalités du travail interprétatif et enfin les jeux de pouvoir entre les acteurs. Plus fondamentalement, notre modèle articule trois traditions de pensée à l'oeuvre dans les théories des organisations et, plus largement, dans les sciences sociales. La première est celle qui analyse les structures organisationnelles, en particulier le courant de la contingence structurelle, avec des auteurs comme March et Simon, Galbraith ou encore Mintzberg, dont on a rappelé les apports en début d'exposé. Nonobstant leurs limites, ces travaux ont le mérite d'avoir mis en lumière la variété des mécanismes de coordination du travail, dont les standards. Comme on l'a montré plus haut, ce courant établit volontiers des liens de cause à effet, par exemple entre les 
caractéristiques du contexte et les modalités de la coordination; il privilégie donc ce que Berthelot dénomme le schème causal (1990, pp.62-65). La deuxième tradition est celle qui analyse ce que nous avons appelé le travail d'interprétation - l'enactment de Weick. Elle renvoie plus largement à ce que Berthelot appelle le schème herméneutique (pp.72-76). La troisième tradition recouvre l'analyse des relations de pouvoir entre acteurs - voir nos emprunts à des auteurs comme Crozier et Friedberg, ou Reynaud. Elle renvoie à un schème plus actantiel (pp.7681).

Cette intégration de trois modes de pensée contribue également à diversifier et à articuler les voies d'action potentielles. Suivant que l'on privilégie les structures, les interprétations ou le pouvoir, on voit en effet se dégager des possibilités d'action spécifiques pour faire face aux situations extrêmes. En cas de relations de pouvoir asymétriques, l'action managériale aura sans doute plus d'impact sur la coordination effective des activités si elle se concentre sur des dispositifs écrits et/ou matériels: le travail interprétatif y est en effet généralement de faible ampleur, voire exceptionnel. Dans le cas de relations de pouvoir plus symétriques, elle gagnera probablement en pertinence en veillant à réunir les conditions favorables au travail interprétatif : lieux institués de débat, dégagement du temps nécessaire à la maturation collective des décisions, soutien logistique au travail de documentation et à l'enrichissement ex post des standards, valorisation symbolique du travail interprétatif réalisé, etc. On remarquera que notre réflexion aboutit à " encadrer » le schème causal traditionnel de la pensée contingente et le schème herméneutique de l'école du sensemaking et de l'enactment par un schème actantiel, dont l'influence s'avère finalement cruciale. C'est en effet le caractère plus ou moins symétrique des relations entre acteurs qui semble conditionner la pertinence des dispositifs managériaux utilisés pour faire face aux situations extrêmes. En outre, la modification des rapports de pouvoir (via le relâchement ou, au contraire, le renforcement du contrôle hiérarchique, par exemple) paraît avoir des conséquences concrètes sur l'intensité du travail interprétatif susceptible d'être opéré.

De telles implications managériales, qui sont évidemment à valider lors de recherches ultérieures, ouvrent néanmoins des pistes tout à fait nouvelles pour la gestion et la coordination des activités en situation extrême.

\section{Références}

Adrot A. et Garreau L. (2010), « Interagir pour improviser en situation de crise », Revue française de gestion, vol.4, $\mathrm{n}^{\circ} 203$, p. 119-131.

Basbøll T. (2010), "Softly Constrained Imagination: Plagiarism and Misprision in the Theory of Organizational Sensemaking ", Culture and Organization, vol.16, n², p.163178, June.

Berthelot J.-M. (1990), L'intelligence du social, Presses Universitaires de France, Paris, Coll. « Sociologie d'aujourd'hui ». 


\section{L'interprétation des standards en situation extrême : le pouvoir fait-il la différence?}

Bigley G. et Roberts K. (2001), " The Incident Command System: High Reliability Organizing for Complex and Volatile Task Environments ", Academy of Management Journal, vol.44, n6, p.1281-1299.

Blau P.M. et Scott W.R. (1962), Formal Organizations, Scott-Foresman, San Francisco, (Ca).

Crozier M. et Friedberg E. (1977), L'acteur et le système. Les contraintes de l'action collective, Seuil, Paris, Coll. "Sociologie Politique ".

DiMaggio P. et Powell W. (1983) «The Iron-Cage Revisited: Institutional Isomorphism and Collective Rationality in Organizational Field », American Sociological Review, ${ }^{\circ}{ }^{\circ} 8$, p.147-160, April.

Faraj S. et Xiao Y. (2006), « Coordination in Fast-Response Organizations », Management Science, vol. 52, n8, p.1155-1169.

Galbraith J.R. (1973), Designing Complex Organizations, Addison-Wesley, Reading.

Godé-Sanchez C. (2010a), « Se coordonner en environnement volatil : les pratiques de coordination développées par les pilotes de chasse ", Finance, Contrôle, Stratégie, vol. $13, n^{\circ} 3$, p. 61-93.

Godé-Sanchez C. (2010b), « Leveraging Coordination in Project-Based Activities: What Can We Learn From Military Teamwork? », Project Management Journal, vol. 41, n³, p. 69-78, June.

Lawrence P.R. et Lorsch J.W. (1989), Adapter les structures de l'entreprise: intégration ou différenciation, 1ère édition américaine en 1967, Éditions d'Organisation, Paris.

Lert F. (2009), Pilotes en Afghanistan. Des aviateurs au combat..., Alti Press, Paris.

March J.G. et Simon H.A. (1991), Les organisations: problèmes psycho-sociologiques, 1 ère édition américaine en 1958, Dunod, Paris.

Merton R.K. (1960), "Bureaucratic Structure and Personality ", Social Forces, n¹8, p.560-568.

Mintzberg H. (1982), Structure et dynamique des organisations, 1ère édition américaine en 1979, Éditions d'Organisation/Agence d'Arc, Paris/Montréal.

Morel C. (2005), "L'enfer des boutons. Essai sur une interface homme-machine ordinaire », Annales des Mines. Gérer et comprendre, ${ }^{\circ} 80$, p.77-91, juin.

Ortmann G. (2010), " On Drifting Rules and Standards », Scandinavian Journal of Management, Volume 26, Issue 2, p.204-214, June.

Oswick C. et Roberston M. (2009) « Boundary Objects Reconsidered: from Bridges and Anchors to Barricades and Mazes ", Journal of Change Management, vol.9, n², p.179193.

Pichault F. et Nizet J. (2000), Les pratiques de gestion des ressources humaines, Seuil, Paris, Coll. " Points - Essais ».

Randall J. et Procter S. (2008), « Ambiguity and Ambivalence: Senior Managers' Accounts of Organizational Change in a Restructured Government Department ", Journal of Organizational Change Management, vol.21, $\mathrm{n}^{\circ} 6, \mathrm{p} .686-700$.

Reynaud J.-D. (1997), Les règles du jeu. L'action collective et la régulation sociale, Armand Colin, Paris.

Rico R., Sanchez-Manzanares M., Gil F. et Gibson C. (2008), « Team Implicit Coordination Processes: A Team Knowledge-Based Approach », Journal of Management Review, vol. 33, $n^{\circ} 1$, p. 163-184.

Sapsed J. et Salter A. (2004) " Postcards from the Edge: Local Communities, Global Programs and Boundary Objects », Organization Studies, vol.25, n9, p.1515-1534.

Thévenot L. (1986) « Les investissements de forme », in Thévenot, L. (Ed.), Conventions économiques, Presses Universitaires de France, Paris, Coll. « Cahiers du Centre d'Etudes de l'Emploi », p.21-71.

Thompson J.D. (1967), Organizations in Action: Social Science Bases of Administrative Theory, Mc Graw-Hill, New York. 
Yakura E. K. (2002) « Charting Time: Timelines as Temporal Boundary Objects », Academy of Management Journal, vol.45, n5, p.956-970.

Van de Ven A.H., Delbecq A.L. et Koenig R. (1976), « Determinants of Coordination Modes within Organizations », American Sociological Review, vol. 41, no. 2, p. 322-338.

Weick, K. E. (1995) Sensemaking in Organizations. Sage, London. 


\title{
Construire le sens par le retour d'expérience: le cas de l'Equipe de Voltige de l'Armée de l'air 245
}

\author{
par Cécile Godé 246
}

\begin{abstract}
Résumé
S'inscrivant dans la thématique générale de la coordination inter-individuelle, cet article s'attache à comprendre la contribution du retour d'expérience à la construction collective de sens dans les équipes évoluant en environnement extrême. La recherche repose sur une étude de cas réalisée près de membres de l'Equipe de Voltige de l'Armée de l'Air. Nos résultats suggèrent que la combinaison des processus de communication, d'apprentissage et de socialisation, qui caractérise le retour d'expérience, nourrit la capacité d'un collectif à produire une interprétation consensuelle d'une situation, favorisant ainsi la coordination.
\end{abstract}

Abstract

Addressing the general question of inter-individual coordination, this article aims at exploring how past experience contribute to sense-making when teams interact under extreme circumstances. It relies on a case-study related to the French air force aerobatic team. Our results suggest that the articulation of communication, learning and socialization processes, which characterizes lessons learned, nurtures team's ability to produce consensual interpretation of the situation, promoting coordination at end.

Depuis une dizaine d'années, de nombreuses contributions défendent l'idée que les équipes se coordonnent plus efficacement lorsque leurs membres s'accordent sur une interprétation possible de la situation (Berman et al., 2002 ; Klein et al., 2005 ; Faraj et Xiao, 2006; Rico et al., 2008; Foss et Lorenzen, 2009 ; GodéSanchez, 2010a, Godé-Sanchez, 2010b). Une telle perspective fait directement référence aux travaux de Weick (Weick, 1979 ; Weick, 1993 ; Weick 1995), qui démontrent comment la construction collective de sens favorise la coordination inter-individuelle. S'ils n'ont pas nécessairement besoin de partager les mêmes valeurs ou les mêmes aspirations (Weick, 1979, Allard-Poesi, 2003), les individus doivent être capables de parvenir à un consensus sur les moyens à mettre en œuvre pour accomplir une ou plusieurs actions. En cela, la construction collective de sens évoquerait un processus clé de coordination dans les équipes.

245. A travers cet article, l'auteur veut rendre hommage au capitaine Renaud Ecalle, membre de l'Equipe de Voltige de l'Armée de l'air et champion du monde 2009, disparu tragiquement avec sa famille le 3 octobre 2010.

246. CÉCILE Godé, Chercheur au Centre de Recherche de l'Armée de l'Air (CReA), GREDEG UMR 6227 CNRS, Université de Nice Sophia-Antipolis, cecile.gode@inet.air.defense.gouv.fr 
Se pencher sur ce processus implique de poser la question du comment : comment le sens émerge-t-il, se développe-t-il ou encore comment s'effondre-til. II s'agit alors d'accorder une importance particulière aux ressources déployées par les individus pour construire ou reconstruire le sens. Dans la perspective interactionniste retenue par Weick, l'élaboration de sens repose sur une dynamique récursive qualifiée d'énaction : les actions et interactions affectent les structures sociales qui, simultanément, pèsent sur elles soit en les contraignant, soit en les « habilitant » (Giddens, 1984). Comme le précise Koenig (2003) : «l'acteur produit autant l'environnement qu'il est produit par lui » (p. 19). Dans ce cadre, l'analyse des ressources et moyens exploités par les individus dans le processus d'élaboration de sens ne peut être dissociée de la "situation » dans laquelle ils se trouvent, à savoir des éléments sociaux, organisationnels, spatiaux et temporels caractérisant le contexte de l'action collective (Girin, 1990 ; Journé et Raulet-Crozet, 2008).

Le plus souvent, la question du comment s'élabore le sens dans les équipes est abordée à travers l'analyse des modes de communication entre les membres (Weick, 1993, Quinn et Duton, 2005 ; Eisenhardt, 1993), du mécanisme d'apprentissage par essai et erreur (Ron et al, 2006) ou encore des activités de socialisation (Berman et al., 2002). Dans cet article, nous nous intéressons à un moyen de construire collectivement le sens encore peu exploré par la littérature : le retour d'expérience. Evoquant un travail de rétrospection d'une action passée afin de concevoir l'action à venir, le retour d'expérience renvoie aux trois dimensions de communication, de socialisation et d'apprentissage communément étudiées par la littérature. Cet article s'attache donc à comprendre la contribution du retour d'expérience à la construction collective de sens dans les équipes. A l'instar de certains auteurs s'intéressant à la question du sens comme processus de coordination (Weick, 1993 ; Klein et al., 2005 ; Faraj et Xiao, 2006 ; Godé-Sanchez, 2008 ; Godé-Sanchez, 2010a), nous choisissons d'examiner les actions et interactions en environnement extrême (Lièvre et Gauthier, 2009 ; Pichault et al., 2010). Comme indiqué dans l'introduction de ce numéro spécial, il s'agit de jouer sur les effets paroxysmiques qui y sont associés afin de mettre à jour des pratiques collectives difficilement observables lors de situations de gestion plus classiques.

L'article se scinde en trois parties. Après avoir défini le caractère extrême d'une situation, la première partie précise tout d'abord en quoi l'élaboration collective de sens est essentielle à la coordination pour ensuite introduire la question de la place du retour d'expérience dans ce processus. La deuxième partie offre un contenu empirique aux propositions théoriques à travers l'étude des pratiques de retour d'expérience développées par les membres de l'Equipe de Voltige de l'Armée de l'air (EVAA). La troisième section tire des enseignements de l'étude de cas, modélisant la façon dont le retour d'expérience nourrit la capacité d'un collectif à produire une interprétation consensuelle d'une situation. 


\section{Construire le sens par le retour d'expérience: le cas de l'Equipe de Voltige de l'Armée de l'air}

\section{La construction collective de sens, un processus clé de coordination en environnement extrême}

Cette première partie aborde le phénomène de coordination en environnement extrême en insistant sur les notions de construction collective de sens et de retour d'expérience.

\subsection{Se coordonner en environnement extrême : l'importance de la construction collective de sens}

En termes de coordination, les situations extrêmes (telles que définies en introduction générale, Lièvre et Gautier, 2009, Pichault et al., 2010) se gèrent différemment de situations de gestion plus classiques. En effet, les acteurs ne peuvent plus entièrement se reposer sur les structures formelles et les modèles d'action standard de l'organisation car les circonstances de travail sont instables, les informations ambiguës et les interdépendances changeantes (Faraj et Xiao, 2006). Dans ces circonstances, confrontés à un évènement inattendu, les membres d'une équipe peuvent être conduits à l'interpréter différemment et, par conséquent, à ne plus s'entendre sur les moyens à mettre en œuvre pour atteindre l'objectif. Weick (1979) introduit la notion d'équivocité pour décrire de telles situations et considère la construction (ou reconstruction) collective de sens comme un processus de réduction de l'équivocité : il s'agit pour les acteurs de trouver un consensus sur une interprétation possible de l'évènement afin de pouvoir à nouveau s'accorder sur les actions à entreprendre. Dans ces circonstances, la coordination inter-individuelle résulte d'une dynamique d'attribution collective de sens, fondée sur la capacité des membres de l'équipe à non seulement construire du sens à partir de chacune des situations qu'ils rencontrent, mais à le conserver (ou le reconstruire) lors des phases de basculement vers l'imprévu.

Les auteurs parlent d'intersubjectivité (Weick, 1995), d'esprit collectif (Weick et Roberts, 1993 ; Bigley et Roberts, 2001), de recherche de cohérence (Alsène et Pichault, 2007) ou encore de socles commun de connaissance tacite (Rico et al., 2008) pour décrire les processus à l'œuvre dans la recherche d'une interprétation consensuelle d'une situation. Ces notions renvoient à la question des moyens et ressources exploités par les acteurs pour construire le sens. En l'occurrence, ces contributions concentrent principalement leur attention sur les mécanismes de communication (ex. langage, vocabulaire, communication non verbale), d'apprentissage par essais et erreurs (ex. rôle de l'échec) et de socialisation (ex. activités de cohésion, dynamiques communautaires). Régulièrement évoqué mais encore peu approfondi par cette littérature, le retour d'expérience semble pourtant mériter une attention particulière dans la mesure où il combine ces trois mécanismes. 


\subsection{Le retour d'expérience pour construire collectivement le sens?}

Le retour d'expérience peut se définir comme un travail d'analyse rétrospective d'une action passée afin de concevoir l'action à venir. II permet de capitaliser l'expérience individuelle et collective en favorisant le partage des bonnes pratiques. En cela, le retour d'expérience ne se concentre pas seulement sur l'étude des erreurs passées. II doit être plus largement considéré comme une démarche d'analyse de toutes expériences issues des activités individuelles et collectives. Le retour d'expérience favorise ainsi la mémorisation des connaissances et des compétences individuelles et collectives (Bès, 1998 ; Faure et Bisson, 2000 ; Gilbert, 2001). A la différence d'une simple démarche d'évaluation des activités du passé, le retour d'expérience implique un apprentissage s'exprimant selon des temporalités différentes (boucles courte et longue), à la fois aux niveaux individuel et collectif.

Au sein des équipes évoluant en environnement extrême, le retour d'expérience nécessite une implication forte des membres dans les processus de communication, d'apprentissage et de socialisation. De communication tout d'abord car la spécificité des pratiques métier et l'urgence/dangerosité des situations requièrent a minima le partage d'un langage commun par les acteurs (Bastien et Hostager, 1992 ; Eisenhardt, 1993 ; Godé-Sanchez, 2010a). Celui-ci peut s'articuler autour d'un vocabulaire particulier et/ou de modes de communication plus informels, tel que le langage gestuel. Ensuite, le retour d'expérience repose principalement sur les processus d'apprentissage par essai et erreur (Ron et al., 2006). L'essai et l'erreur représentent la capacité d'un individu et/ou d'un groupe à reconnaître l'inefficacité d'une décision ou d'une procédure, et à en tirer les enseignements pour ne pas la reproduire. Enfin, le retour d'expérience réclame une implication importante des acteurs dans certains processus de socialisation (Van Maanen et Schein, 1979 ; Delobbe et al., 2005). De ce point de vue, la cohésion et l'esprit communautaire jouent un rôle prépondérant. Plus encore, le retour d'expérience, en favorisant le partage des responsabilités et des connaissances, est susceptible d'alimenter à son tour l'esprit communautaire.

La deuxième partie s'attache à éprouver empiriquement ces premières réflexions théoriques à travers l'examen des pratiques de retour d'expérience développées par les membres de l'Equipe de voltige de l'Armée de l'air.

\section{La construction collective de sens au sein de l'Equipe de Voltige de l'Armée de l'air}

Notre travail s'appuie sur une étude de cas réalisée près de membres de l'Equipe de voltige de l'Armée de l'air (EVAA) de juin à septembre 2010. Après avoir 


\section{Construire le sens par le retour d'expérience : le cas de l'Equipe de Voltige de l'Armée de l'air}

présenté le contexte de la recherche ainsi que la méthodologie adoptée pour recueillir et traiter les données, nous exposons les résultats de l'analyse du cas.

\subsection{Contexte de la recherche}

L'Equipe de Voltige de l'Armée de l'air (EVAA) est située sur la base aérienne 701 de Salon de Provence. Elle se compose de cinq pilotes et de neuf mécaniciens. Outre le fait que les pilotes aient bénéficié de vols de voltige lors de leur formation initiale, ils proviennent tous de l'aviation de chasse et possèdent une expérience opérationnelle importante dans leur métier d'origine ${ }^{247}$. Pour autant, ils s'accordent à considérer la voltige comme une discipline à part, très éloignée des métiers de la chasse. Un pilote explique : "La voltige aérienne, c'est très particulier : on s'envole avec une laisse qui fait un $\mathrm{km}$ de long [le volume d'évolution du voltigeur, cf. figure 1] ". Un autre précise : "Ce qu'on fait à l'EVAA, c'est vraiment spécifique par rapport aux escadrons. En fait, arriver à l'EVAA, ça signifie arrêter son métier précédent pour en commencer un nouveau : la voltige ». Cette discipline est considérée comme un sport physiquement très exigeant, les pilotes subissant des facteurs de charge (positif et négatif) montant régulièrement jusqu'à 10 « $G$ ».

Aujourd'hui, l'équipe est constituée de pilotes nouvellement arrivés, possédant une expérience (disparate) de la voltige de compétition nationale et/ou internationale, ainsi que de pilotes plus anciens, qui se sont forgés leur expérience au fil des années passées à l'EVAA. Le commandement de l'équipe est assuré par un des cinq pilotes. Les autres sont susceptibles de prendre en charge des activités dites " annexes » (communication extérieure, par exemple), qu'ils réalisent en sus des activités de pilotage. Travaillant en étroite collaboration avec les pilotes, les mécaniciens ont en charge la mise en œuvre et la maintenance des trois avions de voltige Extra 330 de l'EVAA. A tour de rôle, ils accompagnent les pilotes lors de leurs fréquents déplacements afin de s'occuper des aspects mécaniques et logistiques. Tout comme les pilotes, les mécaniciens proviennent d'escadrons de l'Armée de l'air, au sein desquels ils ont acquis une expérience importante sur avion (de chasse, de transport ou léger).

L'équipe de voltige doit conduire à bien deux missions. Tout d'abord, elle intervient dans les meetings aériens militaires et civils. Les meetings ont principalement lieu durant la période estivale, la période hivernale étant réservée à l'entraînement. La plupart du temps, les déplacements impliquent deux pilotes et un mécanicien. Les pilotes présentent au public un programme dit « libre intégral », consistant en un enchaînement de figures libres (elles ne sont soumises à aucune règle, exception faites des règles de sécurité) illustrant leur expertise et leur savoir faire, ainsi que la manoeuvrabilité de l'avion.

247. L'aviation de chasse se compose de trois métiers différents, fondés sur des modes d'action et des procédures spécifiques la défense aérienne (offensive et défensive), le bombardement (en profondeur et rapproché) et le bombardement stratégique (qui correspond aux frappes aériennes stratégiques). Actuellement, les pilotes de l'EVAA sont issus des trois métiers de la chasse. 
Ensuite, l'EVAA participe aux compétitions organisées au niveau national et international. Ces deux dernières années, l'équipe a obtenu les meilleurs résultats possibles. Elle est en effet revenue avec la médaille d'or des championnats du monde Unlimited (catégorie la plus élevée) à Silverstone en 2009. En individuel, les pilotes ont remporté la première, la troisième et la cinquième place. L'ensemble de ces titres sera remis en jeu en 2011. Concernant le championnat de France catégorie Unlimited 2010, l'EVAA est revenue avec la première et la deuxième place. Les derniers résultats au championnat d'Europe (septembre 2010) confirment ce niveau d'excellence avec une première place en individuel et une deuxième en équipe. En compétition, les pilotes exécutent des programmes imposés (connu - les pilotes y ont accès plusieurs mois avant la compétition - et inconnu - ils ne prennent connaissance des figures que quelques heures avant le vol) et libres, évalués par une dizaine de juges. Les différents programmes sont réalisés dans un volume d'un $\mathrm{km}^{3}$, appelé « box », et matérialisé au sol par des repères (cf. figure 1). Les juges notent sur 10 la réalisation des figures et des enchaînements, les coefficients appliqués étant directement corrélés au niveau de difficulté proposé. La capacité du pilote à évoluer dans son box (sans en sortir ${ }^{248}$ et en respectant les règles de sécurité du vol) et à en exploiter le volume (par exemple, en évoluant au plus près des juges) est également évaluée.

Figure 1. Le volume d'évolution du voltigeur en compétition : le « Box $»^{249}$

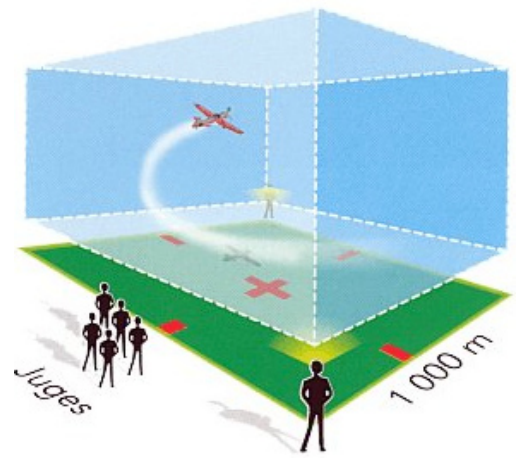

Au regard des activités de présentation, la compétition présente une particularité notable : le périmètre de l'équipe s'étend à des pilotes de voltige civils. Ainsi, l'équipe de France engagée en compétition catégorie Unlimited est aujourd'hui constituée de trois voltigeurs militaires, de trois pilotes civils masculins et deux pilotes civils féminins. La sélection des pilotes est opérée par l'entraîneur national, sous l'autorité du Directeur Technique National de la Fédération Française Aéronautique et du commandant de l'EVAA en ce qui concerne les pilotes militaires.

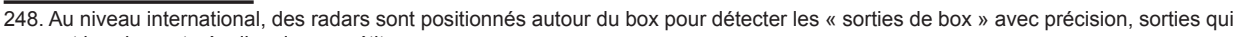
peuvent lourdement pénaliser le compétiteur.

249. Source : http://www.equipedevoltige.org 


\section{Construire le sens par le retour d'expérience : le cas de l'Equipe de Voltige de l'Armée de l'air}

Dans cette équipe à périmètre évolutif, la bonne coordination repose sur la capacité de ses membres à générer collectivement du sens à partir des deux missions différentes qu'ils ont à conduire. Comme le précise Alsène et Pichault (2007), cette construction collective (les auteurs parlent de recherche de cohérence) met l'accent à la fois sur l'importance de l'orchestration des activités (agencement des ressources et des efforts individuels) et sur leur harmonisation (chacun partage la même représentation de l'environnement et s'accorde sur les moyens à mettre en œuvre pour parvenir à l'objectif). Traduit au niveau de l'EVAA, il s'agit d'une part de gérer les aspects logistiques des activités de présentation et de compétition (allocation des personnels, disponibilité des appareils, gestion de la charge de travail des pilotes et des mécaniciens, aménagement des emplois du temps, etc.) et, d'autre part, d'intégrer les connaissances et les savoir-faire de ses membres afin que chacun aille dans le même sens et contribue à l'excellence des résultats en meetings et en compétition.

Les membres de l'équipe de voltige se coordonnent au sein d'un environnement que l'on peut qualifier d'extrême (Lièvre et Gauthier, 2009) : la situation peut être amenée à changer très rapidement (ex. conditions météo instable, une collision volatile, un problème mécanique), l'incertitude est forte quant à la survenance d'un évènement inattendu (la probabilité d'occurrence de l'imprévu est difficile à estimer) et les programmes présentés en vol impliquent un niveau de risque important : un risque physique dans la mesure où la vie des pilotes peut être engagée, mais également un niveau de risque plus symbolique, notamment associé au classement en compétition et au niveau de prestige associé.

Dans ces circonstances, les acteurs sont conduits à construire et maintenir collectivement un sens aux situations auxquelles ils sont confrontés. Nous verrons que le retour d'expérience joue alors un rôle prépondérant. En encourageant les échanges de connaissances et de savoir-faire sur des modes le plus souvent informels, il permet en effet d'impliquer les acteurs dans des cycles d'apprentissage aux niveaux individuel et collectif : au niveau individuel en permettant aux pilotes de progresser techniquement par exemple, et au niveau collectif en impliquant l'ensemble de l'équipe dans le processus d'amélioration.

\subsection{Méthodologie}

Dans cette recherche de nature qualitative, nous avons réalisé une étude de cas extrême (Yin, 2003) afin d'appréhender une situation rarement étudiée en sciences de gestion. L'objet de l'étude de cas est descriptif, au sens où il s'attache à « décrire une intervention et le contexte réel dans lequel elle s'est produite » (Yin, 2003, p. 15). Le cas sur lequel nous nous penchons a été sélectionné en vue d'un enrichissement théorique des concepts de coordination en environnement extrême et d'élaboration collective de sens. II s'agit en fait de construire un échantillon théorique, en considérant les éléments de théorie développés précédemment 
comme des critères de sélection du cas. La généralisation analytique s'en trouve ainsi facilitée (Ayerbe et Missonier, 2007).

Le corpus des données de terrain a été recueilli durant le mois de juin. II a été construit par triangulation (1) d'entretiens semi structurés, (2) d'observations in situ et (3) de documents internes écrits et vidéo (Eisenhardt, 1989) :

(1) sur la base aérienne de Salon de Provence, j'ai conduit six entretiens individuels semi structurés d'une heure chacun environ auprès de quatre pilotes (dont le chef de l'équipe et le champion du monde de voltige), d'un mécanicien et de l'entraîneur de l'équipe de France. Le guide utilisé était structuré autour des thématiques du retour d'expérience et de la coordination au sein de l'équipe.

(2) l'observation non participative de sept vols d'entraînement de l'équipe de France de voltige catégorie Unlimited m'a permis d'approfondir la réalité des pratiques évoquées en entretiens. Ce jour là, les pilotes devaient réaliser un programme dit « inconnu », dont ils avaient eu connaissance la veille au soir (les vols s'effectuant de $9 \mathrm{~h} 30$ à 12h00). J'ai pu observer des séances de « musique » réalisées par certains pilotes juste avant leur départ en vol et leur permettant de se concentrer par imagerie mentale. Un des pilotes m'a donné le programme Aresti (cf. figure 2) et m'en a explicité les figures à partir du premier vol d'entraînement. J'ai ensuite écouté les commentaires (off et en direct) de l'entraîneur sur chacun des vols. J'ai pris des notes et ai pu transcrire certains passages. Enfin, j'ai eu l'opportunité de visionner plusieurs films extérieurs, enregistrés du point central, et intérieurs, montrant les gestuels des pilotes, leur façon de piloter et d'endurer les facteurs de charge. Cette possibilité d'observer une situation singulière m'a permis d'appréhender l'enchaînement des pratiques constitutives de la coordination et du retour d'expérience au sein de l'équipe (Lièvre et Rix-Lièvre, 2009).

(3) enfin, j'ai réalisé une collecte d'articles de presse et de reportages dédiés aux résultats de l'équipe et des pilotes au championnat du monde. Le site Web de l'EVAA, différents forums de discussions ainsi que les plaquettes de communication m'ont également été très utiles pour mieux comprendre les différentes activités des membres de l'équipe et les enjeux associés.

Le traitement des données de terrain a été réalisé par codage ouvert afin de faire émerger les thèmes représentatifs et réguliers au fil de l'analyse. La souplesse d'usage du logiciel NVivo7 a permis de révéler de nouveaux thèmes tout au long du traitement du matériel, éprouvant la robustesse de la première liste de codage. La section suivante se reporte à cette liste de codage pour exposer les résultats de terrain. 


\section{Construire le sens par le retour d'expérience: le cas de l'Equipe de Voltige de l'Armée de l'air}

\subsection{Résultats de terrain}

Opéré dans la perspective de mieux comprendre la contribution du retour d'expérience à la construction collective de sens, le codage des données de terrain révèle deux catégories thématiques principales : la nature (informelle et à boucle courte) du retour d'expérience (1) ainsi que ses qualités cohésives (2).

\subsubsection{Un retour d'expérience informel et à boucle courte}

A la question : "Spontanément, que vous évoque le retour d'expérience ? ", les membres de l'Equipe de Voltige de l'Armée de l'air (EVAA) interviewés convergent tous vers une même réponse : la transmission d'un savoir d'un type particulier. Un pilote indique : "Ici, on ne parle pas de savoirs théoriques. On parle de sensations, du 'pilotage aux fesses', comme on dit ». Un autre précise : " nous sommes dans le pilotage pur, où les sensations sont essentielles. Par exemple, à la question : ou mettre le pied pour faire un déclenché ? vous ne trouverai pas la réponse dans une base de données ! La connaissance théorique, c'est bien... mais elle n'a pas de valeur tant qu'elle n'est pas mise en pratique».

Marqué par la nature expérientielle du savoir qu'il permet de transmettre, le retour d'expérience à l'EVAA repose sur des pratiques spécifiques, relativement éloignées des procédés communément mis en œuvre durant les débriefings. En effet, alors que les pilotes se soumettent à des procédures de retour d'expérience formalisées et très standardisées en escadrons de chasse (Ron et al., 2006), les membres de l'équipe de voltige ont développé une façon de faire à part, caractérisée par ses aspects informel (1) et à chaud (2).

(1) D'une part, la transmission des connaissances est principalement réalisée à partir de discussions et de dialogues informels, en face à face ou en groupe : "l'expérience, on ne la trouve pas dans un classeur ; elle se transmet à l'oral et elle se vit ».Ces échanges impliquent les pilotes (militaires et civils) et l'entraîneur pour tous les vols de compétition (stages équipe de France), les pilotes et les mécaniciens en ce qui concerne les activités de meeting. Un pilote explique : « le retour d'expérience, c'est principalement le bouche à oreille entre nous, qu'on soit civil ou militaire. Je pense sincèrement que le retour d'expérience le plus efficace, il se fait grâce à la communication entre les hommes. Le papier, la vidéo... tout ça ne représente que des outils qui ne permettent qu'une transmission partielle du savoir qu'on a accumulé année après année ».

Cette " tradition orale » du retour d'expérience est rendue possible tout d'abord grâce à une certaine forme de standardisation du langage. Les membres de l'équipe partagent en effet un langage commun qui emprunte à la fois au vocabulaire de l'aéronautique générale et à un code spécifique des voltigeurs : le code Aresti (transcription schématique des figures de voltige, cf. 
figure 2). Ce langage standardisé, loin d'entraver les discussions informelles entre les membres, les facilite en leur permettant d'aller à l'essentiel, sans s'embarrasser de longues descriptions introductives. Un pilote indique : " dans l'équipe, on parle tous le même langage, certainement parce qu'on partagent les connaissances et valeurs de l'aéronautique (militaire et civile). Je pense que c'est la raison pour laquelle le retour d'expérience se fait naturellement et rapidement entre les membres ". II apparaît ensuite que certains espaces favorisent plus que d'autres ces pratiques informelles du retour d'expérience. C'est notamment le cas de la salle de repos, qui représente un espace privilégié de transmission des expériences, qu'elles soient récentes ou plus anciennes. Un mécanicien raconte : "ici, on se retrouvent tous dans un même espace, le matin, le soir. Et il y a beaucoup de choses qui se disent au café. Par exemple, quand on revient de meeting nous, les mécanos, on est parti seuls avec les pilotes et on commence à en parler aux autres [ceux qui sont restés] de façon informelle : il faut mettre en place tel réglage avec tel pilote pour éviter d'aggraver sa tendinite, etc. ". A la différence des escadrons de chasse classiques, la salle de repos de l'EVAA est unique, permettant à l'ensemble des membres de l'équipe de se réunir : pilotes, mécaniciens et personnel administratif savent qu'ils vont s'y retrouver quotidiennement et pouvoir profiter de l'occasion pour discuter. Le mécanicien continue : "dans la salle de repos de l'escadron se résolvent énormément de problèmes. Ça nous permet de diffuser aux bonnes personnes, en discutant avec elles. C'est pour ça que les espaces où les gens se réunissent et peuvent communiquer librement, c'est essentiel! ». Ce faisant, le retour d'expérience s'enrichit des points de vue de l'ensemble des métiers, les uns étant étroitement liés aux autres et participant de la réussite des pilotes en meeting et compétition : "les cultures et les approches sont différentes et le collectif a beaucoup à gagner des échanges d'expériences des personnels issus des différents métiers composant l'EVAA ». 


\section{Construire le sens par le retour d'expérience : le cas de l'Equipe de Voltige de l'Armée de l'air}

Figure 2. Exemple d'un programme simple de voltige schématisé en code Arestij ${ }^{250}$

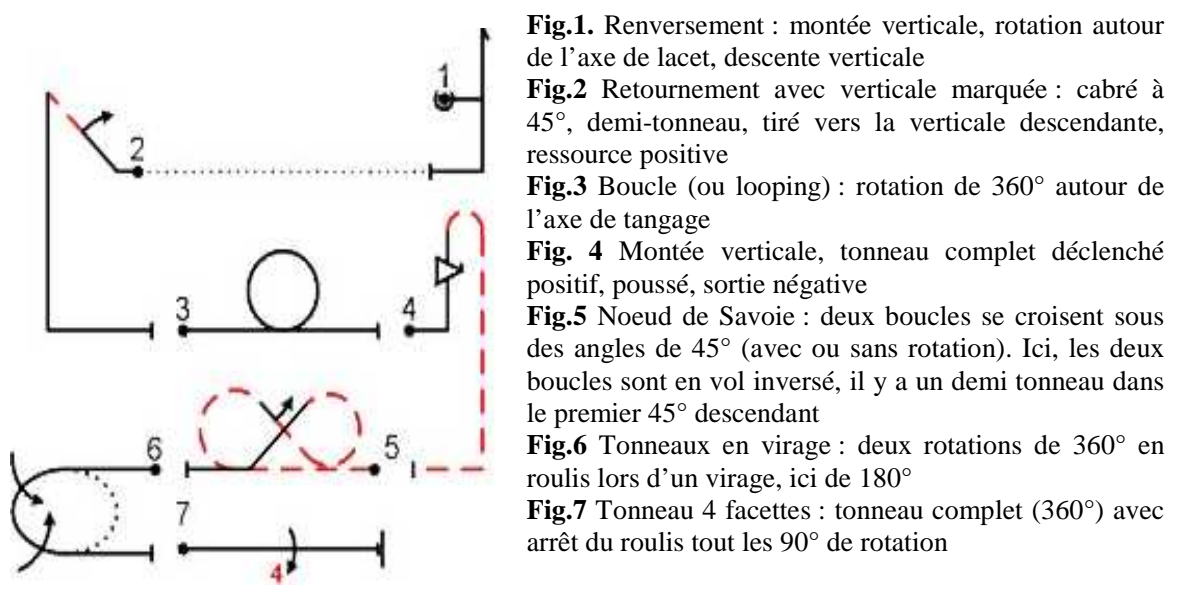

(2) D'autre part, les pratiques de retour d'expérience au sein de l'EVAA se font principalement " à chaud ». Comme le précise un des pilotes interviewés : " la diffusion est assez immédiate. Nous effectuons des vols courts, d'environ 15 minutes, et le retour d'expérience se fait soit pendant que nous volons, soit juste après le vol ». Dans le premier cas, il est fait référence à un deuxième voltigeur (un des pilotes de l'équipe de voltige ou l'entraîneur) situé au point central, c'est-à-dire dans une zone où il aura un bon visuel du programme effectué. Systématiquement, un caméraman l'accompagne pour filmer le vol. La personne au point central est en liaison radio avec celui en évolution, pouvant ainsi lui faire ses commentaires (et critiques) en direct. Un autre pilote explique : « les pilotes de voltige ont besoin de la voix de l'entraîneur qui scande les figures, critique sévèrement ce qu'ils sont en train de faire, tout ça en temps réel; ça nous permet de mettre immédiatement en pratique le retour d'expérience, de refaire la figure encore et encore... ».

Le point central peut également enregistrer ses commentaires " en off »; ils accompagneront alors la vidéo du vol. II s'agit là du deuxième type de retour d'expérience à chaud : juste après l'atterrissage, le pilote se rend dans une petite salle de projection où il visionne son vol. Comme l'explicite l'entraîneur, il n'est alors plus dans l'action, mais dans la réflexivité : " je fais des commentaires en voix off, que le pilote n'entendra qu'une fois le vol terminé, assis devant son film. II scrute sa prestation, observe les erreurs qu'il a commises et doit également se rendre compte de ce qu'il a bien fait. Pourquoi il a réussi cette figure et pourquoi il a échoué sur celle-ci sont des réponses qu'il doit obtenir, avec mon aide ou celle d'un collègue si besoin. II doit prendre du recul pour appréhender son état d'esprit aux moments clés du vol ; il doit apprendre à se connaître pour progresser. Ça passe par l'auto-

250. Source : http://www.equipedevoltige.org 
critique et par l'acceptation des critiques (constructives !) des autres ». Un système vidéo peut également être installé à l'intérieur du cockpit, la plupart du temps dirigé vers les pieds et/ou les mains du pilote. Lors du visionnage, celui-ci observe ses "façons de faire » en situation. Ces séances d'autoconfrontation, souvent réalisées en présence de tiers, permettent de mettre à jour les petites erreurs techniques, sources de problèmes en vol : « à partir de ces films intérieurs, le pilote et moi-même constatons la façon dont il pilote. Par exemple, le circuit visuel est très important en voltige, et souvent les pilotes ne savent pas où regarder. Ils ont du retard dans le circuit visuel, ce qui leur fait mal déclencher leurs mouvements, de façon précipitée. II suffirait qu'ils aient une ou deux secondes d'avance sur leur circuit visuel pour qu'ils voient l'objectif arriver et déclenchent avec plus de vivacité. Avec la caméra intérieure, on peut comprendre pourquoi l'anticipation est mal opérée ».

Parallèlement aux pratiques de retour d'expérience «à chaud », principalement consacrées au vol de voltige en tant que tel, les membres de l'équipe sont également impliqués dans des processus d'apprentissage par l'expérience plus longs. Par exemple, les pilotes sont régulièrement invités à participer aux réunions de débriefing à la fin de la saison de représentations. II s'agit de recueillir leurs impressions, commentaires et critiques afin d'améliorer l'organisation des meetings l'année suivante.

\subsection{2. " Je t'aime, moi non plus » : le retour d'expérience, source de cohésion dans une équipe de compétiteurs}

Les pratiques informelles et à chaud du retour d'expérience constituent une source capitale de cohésion au sein d'une équipe tiraillée entre ses objectifs de démonstration d'un savoir-faire collectif en représentation et de résultats individuels en compétition. II y a d'un côté les activités de meetings qui permettent notamment de faire connaître la discipline de la voltige aérienne ainsi que l'Armée de l'air et de faire naître des vocations. Un pilote explique : "lorsqu'on est en meeting aérien, on fait du spectacle. II faut se mettre dans la peau d'un artiste qui cherche à éveiller des émotions ". Plus encore, l'excellence des figures présentées est une garantie de bonne image de la discipline vers l'extérieur, bénéficiant ainsi à la communauté des voltigeurs dans son ensemble. Le même pilote développe : " si l'EVAA n'existait pas, la voltige aérienne en France n'en serait pas à ce niveau là. Par exemple, tous les progrès réalisés sur l'avion actuel [l'Extra 330] ont rejailli sur le monde de la voltige civile. Lorsque on est présents en meeting, on fait connaître la discipline, on attire les médias : qui dit médias dit sponsors, qui dit sponsors dit fonds, etc. ". Les représentations impliquent donc l'équipe en tant qu'entité, chaque vol individuel cristallisant un savoir-faire collectif. Dans ces circonstances, les pilotes ont tout intérêt à s'investir dans le retour d'expérience afin de bénéficier, et de faire bénéficier l'autre, de leurs connaissances pour progresser. Un autre pilote explique : " moi, j’apprends 


\section{Construire le sens par le retour d'expérience: le cas de l'Equipe de Voltige de l'Armée de l'air}

en regardant les vidéos intérieures des autres. Sur un programme connu par exemple, où on sait déjà ce que le pilote va faire, c'est très intéressant pour avoir les bons gestes. Et puis la vidéo du copain, c'est une forte émulation : on voit à quel point il se donne du mal, il encaisse fort et cela peut être une motivation supplémentaire pour se donner davantage soi-même ".

De l'autre côté, il y a la compétition, naturellement marquée par des dynamiques individuelles : " la voltige, c'est un sport individuel. On ne sélectionne pas les gens sur leur capacité à être sympa, mais sur leurs compétences de compétiteurs. Ça veut dire que tout le monde se bat pour la première place. Même si aujourd'hui, on est champions du monde par équipe, on est tous câblés sur les classements individuels. Le résultat par équipe est une conséquence de nos savoir-faire individuels, pas une finalité ». Les pilotes militaires sont aujourd'hui parmi les meilleurs voltigeurs du monde et se concurrencent directement les uns les autres. Ils pourraient alors percevoir le retour d'expérience comme un danger potentiel, dans la mesure où celui-ci favorise la diffusion de connaissances clés pour gagner. Un des pilotes reconnaît cette idée mais la réfute aussitôt : " un pilote qui vole et qui garde toutes les infos pour lui, ça pourrait très bien se concevoir s'il est en lice pour la première place. Dans mon cas, je suis en place de leader actuellement et je me sens bien sûr menacé ! C'est un équilibre instable. Mais faire de la rétention d'informations, cacher des trucs... C'est pas comme ça que j'ai envie de gagner. Je veux continuer à faire du retour d'expérience ». Ce faisant, au-delà d'un processus de transmission des savoirs, le retour d'expérience est également perçu comme un moyen d'élever le niveau des compétiteurs, une façon de se confronter à toujours plus fort que soi. II s'agit ensuite de relever le défi... « Le niveau des pilotes de l'équipe monte en puissance, et ça nous donne une vraie émulation. L'émulation, c'est de l'encouragement, c'est la volonté de faire mieux que l'autre ».

Ainsi, entre les meetings et la compétition, les membres de l'équipe de voltige alternent continuellement des interactions coopératives et quasi-concurrentielles. La coexistence de telles postures a priori opposées semble notamment rendue possible grâce au retour d'expérience. En effet, les pratiques informelles et à chaud développées par les pilotes évoquent un processus d'intermédiation, une sorte de " passerelle » entre l'attitude du compétiteur d'une part et celle du présentateur de l'autre : "le collectif se construit autour d'un équilibre un peu particulier en fait : il y a l'esprit de compétition, très individualiste, qui nous conduit à vouloir battre tout le monde. Puis il y a l'esprit d'équipe, essentiel pour la survie de l'EVAA, qui doit montrer son savoir-faire et communiquer pour continuer à exister. En diffusant les pratiques, la façon dont on réussit telle ou telle figure, les erreurs à ne pas commettre, le retour d'expérience nous permet de doser tout ça ». Plus encore, en facilitant les interactions sociales, ces pratiques suscitent la cohésion : " les dispositifs de retour d'expérience jouent un rôle sur la cohésion du groupe. Tout d'abord parce qu'on voit la façon dont les autres travaillent, leur 
niveau d'expertise et que ça donne confiance. Ensuite parce que ça permet de connaître l'autre au-delà de ses capacités techniques et de ses expertises. Le retour d'expérience nous permet de l'aborder différemment, de mieux comprendre sa personnalité, ses attitudes ».

Comme le précise l'entraîneur de l'équipe de France, la composition de l'équipe affecte directement la nature du retour d'expérience et, plus indirectement, sa capacité à susciter la cohésion : "L'environnement humain, c'est primordial et l'équipe est intéressante quand les membres s'entendent bien et se complètent. Les membres doivent être soudés et cette cohésion, ils la construisent en échangeant notamment sur leurs expériences pro. Par exemple, les pilotes se réunissent souvent pour regarder la vidéo de l'un de leurs camarades. II s'agit là d'un débriefing de groupe sur la prestation d'un seul. Parfois, les critiques sont lourdes mais ils doivent aussi savoir s'encourager. Tout doit être fait dans un esprit constructif. Sinon, c'est pas la peine, le collectif sera nul et les résultats également ».

\section{Discussion}

En illustrant le rôle joué par le retour d'expérience dans la construction collective de sens, l'étude de cas permet d'affiner notre compréhension du phénomène de coordination inter-individuelle en environnement extrême. Nos résultats de terrain soulignent en effet le rôle important joué par les facteurs sociaux et cognitifs de la coordination, allant ainsi dans le sens de récentes contributions sur le sujet (Faraj et Xiao, 2006 ; Foss et Lorenzen, 2009 ; Godé-Sanchez, 2010a). En particulier, ils suggèrent que la combinaison des processus de communication, d'apprentissage et de socialisation, qui caractérise le retour d'expérience, nourrit la capacité d'un collectif à produire une interprétation consensuelle d'une situation, à créer et maintenir le sens (cf. figure 3).

Au sein d'un contexte fortement changeant, où la probabilité d'occurrence d'un événement imprévisible est forte et le risque vital élevé, les individus sont en quête de connaissances actualisées relatives d'une part à la spécificité de leurs tâches au sein du groupe et, d'autre part, aux moyens à mettre en œuvre pour les réaliser. Evoquant des opportunités de réguler le partage des savoirs, le retour d'expérience en boucle courte est une façon d'accéder à cette connaissance. De ce point de vue, les acteurs doivent tout d'abord pouvoir se reposer sur des moyens de communication leur permettant de filtrer les informations pertinentes de celles qui le sont moins au moment de l'action. L'analyse du cas nous permet d'insister sur le rôle joué par le langage commun dans ce processus, en révélant notamment l'importance de la standardisation. Nous remarquons en effet qu'au sein de l'Equipe de voltige, le langage partagé repose sur un ensemble de schémas et de mots codes (cf. figure 2) qui représentent autant de standards 


\section{Construire le sens par le retour d'expérience: le cas de l'Equipe de Voltige de l'Armée de l'air}

vers lesquels les pilotes peuvent se retourner pour élaborer collectivement le sens. Faisant référence à des figures et aux procédés de base d'exécution de ces figures, ces standards traduisent des modes de raisonnement partagés par tous les voltigeurs : un ensemble de références techniques, identitaire (les standards ne font sens qu'au sein de la communauté des voltigeurs) et sociales (ces standards sont mobilisés pour gérer les interactions entre voltigeurs). En cela, le langage commun nourrit une compréhension mutuelle des activités de chacun au sein du groupe : les acteurs s'y réfèrent pour construire leurs actions et gérer leurs interactions. II favorise également un ancrage collectif en alimentant la dimension identitaire et sociale du groupe.

Ensuite, l'analyse du cas suggère que le retour d'expérience en boucle courte se fonde sur un processus d'apprentissage à moyen/long terme : la réflexivité. Cette articulation des échelles temporelles s'exprime de la façon suivante : les itérations sur les pratiques de travail, réalisées juste après l'action, encouragent l'adoption d'une posture critique pérenne. Plus précisément, la remise en doute quotidienne des pratiques individuelles stimule l'émergence de routines réflexives au niveau du collectif. De telles routines se nourrissent également des différents exercices de retour d'expérience sur le long terme réalisés par les membres de l'équipe lors d'occasions diverses (comme les débriefings de fin de saison de meeting, par exemple). Les routines réflexives jouent un rôle clé dans la construction collective de sens : d'une part, elles conduisent naturellement les acteurs à s'interroger sur le sens qu'ils donnent à une situation ; d'autre part, elles les orientent vers des procédés de résolution de problème fondés sur la remise en question, le débat et la recherche de consensus. L'articulation de ces échelles temporelles se retrouve également lorsqu'on observe les différentes boucles d'apprentissage qui se chevauchent : le retour d'expérience en temps réel (pendant le vol) se nourrit des débriefings vidéo et des discussions informelles qui ont lieu tout de suite après le vol ; forts de ces nouvelles connaissances, les pilotes et mécaniciens se retrouvent ensuite en salle de repos, parfois plusieurs heures après le vol, et sont susceptibles de le " rejouer », voire d'élargir les discussions aux dimensions logistiques (tel évènement lors de tel vol de compétition ou en meeting, par exemple) et/ou humaines (telle interaction qui a induit un problème ou au contraire une amélioration dans les relations de groupe, par exemple).

Enfin, le processus de socialisation, dernier constituant du retour d'expérience, met l'accent sur les dynamiques en mesure de soutenir la réflexivité. En effet, les deux modes d'apprentissage mobilisés par les acteurs, reconnaître ses erreurs et en tirer des enseignements sur le moyen/long terme, sont susceptibles d'exercer une forte pression sociale. Dans ce cadre, ils ne sont viables qu'à condition que l'équipe s'inscrive dans une démarche constructive. II ne s'agit pas d'accuser un individu, de l'exclure, ni de négliger les responsabilités collectives ; il s'agit au contraire de créer des dynamiques internes capables de soutenir la réflexivité. De ce point de vue, nous remarquons le rôle central joué par des espaces dédiés 
aux échanges de connaissances sur un mode informel. Dans le cas de l'EVAA, la salle de repos de l'escadron évoque un tel espace : les membres de l'équipe s'y retrouvent pour échanger sur leurs expériences récentes (ce qui s'est passé en meeting ce week-end et qui doit absolument être su des autres pour aller dans le sens de l'efficacité, par exemple) et plus anciennes (les anecdotes individuelles ou collectives qu'il est intéressant de rappeler pour éviter de commettre les mêmes erreurs que par le passé, par exemple). La dynamique d'échange informel des expériences produit des résultats intéressants dès l'instant où l'ensemble des métiers et des spécialités a accès à ces espaces. Les résultats individuels et d'équipe sont le résultat d'une bonne intégration de l'ensemble des connaissances mobilisées au sein de l'équipe et c'est de la confrontation de points de vue, d'expériences et de visions différentes que se construit le consensus collectif.

Figure 3. Le retour d'expérience comme source de construction collective de sens

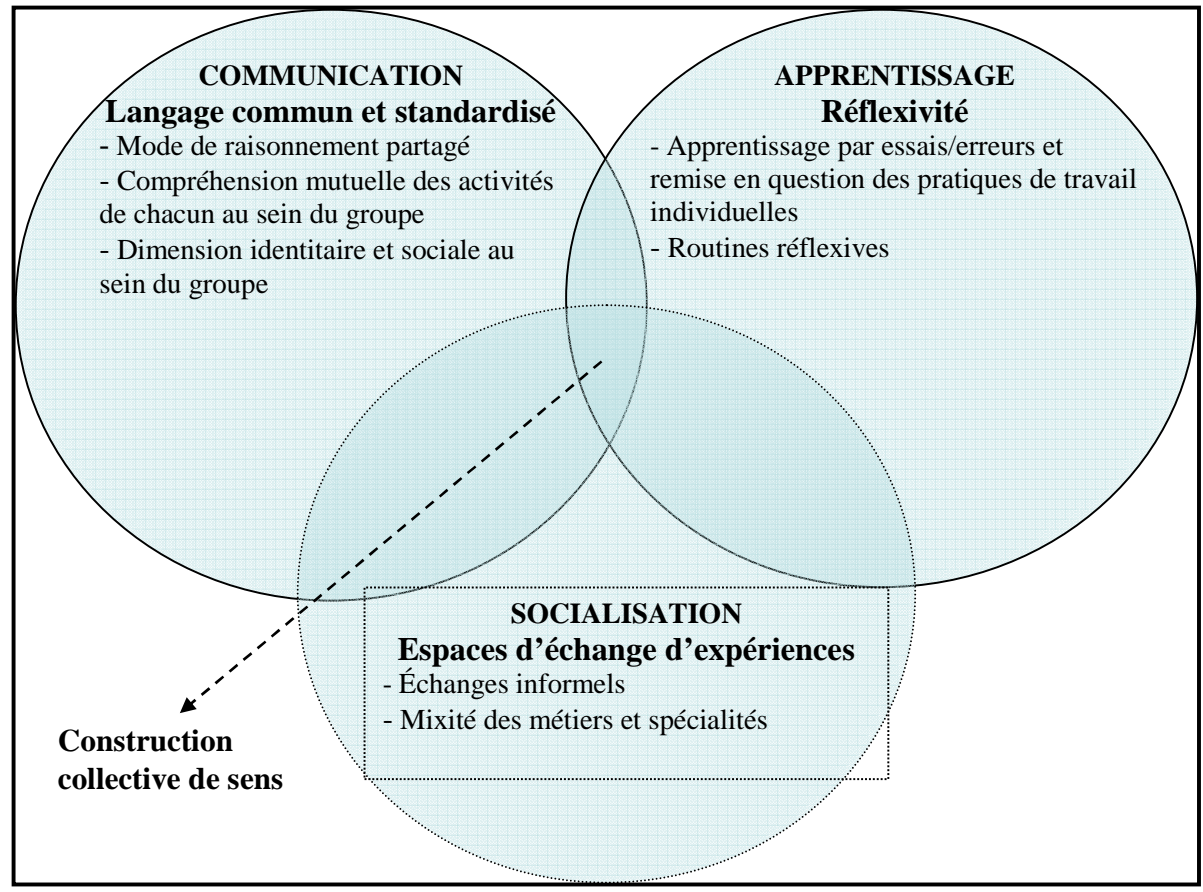

Enfin, l'analyse du cas de l'Equipe de voltige met également en évidence une particularité des équipes de compétiteurs, qui doivent continuellement alterner des interactions coopératives et quasi-concurrentielles (Picq, 2005 ; Sève et al., 2009). En régulant le partage des savoirs, les pratiques de retour d'expérience permettent de rappeler l'existence du collectif comme entité essentielle à l'expression des individualités. Elles évoquent ainsi une passerelle entre l'attitude 


\section{Construire le sens par le retour d'expérience: le cas de l'Equipe de Voltige de l'Armée de l'air}

du compétiteur, qui recherche la première place, et celui du partenaire, qui à la fois aide ses coéquipiers et profite de leurs expériences pour progresser. Dans ces circonstances, le processus de socialisation, en favorisant les échanges informels de connaissances au sein d'espaces dédiés, joue un rôle clé. II permet de rappeler à l'ensemble des membres l'importance du collectif, son esprit, sa culture, ses routines et tous les éléments qui participent de la dynamique constructive dont ils bénéficient en tant qu'individu. Dans une perspective managériale, cette observation conduit à aborder la combinaison des trois processus constituant le retour d'expérience, ainsi que des dispositifs qui les accompagnent, en termes de pondération. II s'agit de trouver le bon équilibre au regard des exigences du moment.

\section{Conclusion}

S'inscrivant dans la problématique générale de la coordination en environnement extrême, cet article a étudié les pratiques de retour d'expérience développées par les membres de l'Equipe de voltige de l'Armée de l'air afin d'appréhender leurs effets sur processus de construction collective de sens. Insistant sur le rôle clé joué par les facteurs sociaux et cognitifs de la coordination, les résultats suggèrent que la combinaison des processus de communication, d'apprentissage et de socialisation nourrit la capacité d'un collectif à produire une interprétation consensuelle d'une situation. Les résultats de terrain permettent également d'avancer l'idée d'une pondération entre ces processus au regard du contexte d'action examiné. En l'occurrence, au sein d'un collectif marqué par les individualités, le processus de socialisation acquiert une importance relative certaine, encourageant notamment les dynamiques interactionnelles et cohésives.

Notre analyse permet d'ouvrir la réflexion sur les jeux de pouvoir associés à la construction collective de sens. En effet, le processus consensuel permettant aux acteurs de s'accorder sur les moyens à mettre en œuvre pour accomplir une ou plusieurs actions nous semble nécessairement affecté par (1) la nature du problème à traiter et (2) le pouvoir relatif des membres de l'équipe au regard de ce problème. Ainsi, dans le cas de l'Equipe de voltige, le niveau d'expertise d'un pilote et la légitimité acquise en compétition lui donneront un poids certain pour toutes les situations réclamant une analyse des pratiques de pilotage et du savoirfaire technique. Cependant, ce pouvoir est transférable vers un autre membre de l'équipe dès l'instant où la nature du problème à traiter évolue. Par exemple, il reviendra au chef d'équipe pour toutes les questions relatives aux orientations stratégiques (recrutement, bâtiment, etc.) prises par l'EVAA. En insistant sur l'importance des relations de pouvoir dans la fabrique de la construction collective de sens, ces dernières remarques viennent nourrir l'analyse précédente (Nizet et Pichault, 2011) considérant les effets des rapports de pouvoir sur le caractère plus ou moins structurant des standards mobilisés par les acteurs en situation. 


\section{Bibliographie}

Allard-Poesi F. (2003), "Sens collectif et construction collectif de sens », in Vidaillet B. (dir.) : Le sens de l'action. Karl E. Weick : socio-psychologie de l'organisation, Vuibert, Série Vital-Roux, Paris, p.91-114.

Alsène E. et Pichault F. (2007), « La coordination au sein des organisations : éléments de recadrage conceptuel », Gérer et Comprendre, N87, p. 61-77.

Ayerbe C. et Missonier A. (2007), « Validité interne et validité externe de l'étude de cas : principes et mise en œuvre pour un renforcement mutuel », Finance, Contrôle, Stratégie, Vol. 10, N², p. 37-62.

Bastien D. et Hostager T. (1992), "Cooperation as communicative accomplishment: A symbolic interaction analysis of an improvised jazz concert", Communication Studies, Vol. 43, p. 92-104.

Berman S.L., Down J. et Hill C.W. (2002), "Tacit knowledge as a source of competitive advantage in the National Basketball Association", Academy of Management Journal, Vol. 45, N ${ }^{\circ} 1$, p. 13-31.

Bès M. P. (1998), « La capitalisation active des connaissances : principes, contextes et obstacles », Gérer et Comprendre, N54, p. 38-51.

Bourgeois L. et Eisenhardt K. (1989), "Strategic decision process in high-velocity environments: Four cases in the microcomputer industry", Management Science, Vol. 34, $\mathrm{N}^{\circ} 7$, p. 816-835.

Delobbe N., Herrbach O., Mignonac K. et Lacaze D. (2005), Comportement organisationnel, volume 1: contrat psychologique, émotions au travail et socialisation organisationnelle, De Boeck, Bruxelles.

Eisenhardt K. (1989), "Building theories from case study research", Academy of Management Review, Vol. 14, Nº, p. 532-550.

Eisenhardt, K. (1993), « High reliability organizations meet high velocity environments: Common dilemmas in nuclear plants, aircraft carriers, and microcomputers firms ", in Roberts K. (dir.): New Challenges to Understanding Organizations, McMillan, New-York, p. 117-135.

Faraj S. et Xiao Y. (2006), "Coordination in fast-response organizations", Management Science, Vol. 52, N8, p. 1155-1169.

Faure A. et Bisson G. (2000), "Gérer les retours d'expérience pour maintenir une mémoire métier, étude chez PSA Peugeot Citroën », Journée Francophone d'Ingénierie des Connaissances, mai, Toulouse, France.

Foss N. et Lorenzen M. (2009), "Towards an understanding of cognitive coordination: Theoretical development and empirical illustrations", Organization Studies, Vol. 30, №11, p. 1201-1226.

Giddens A. (1984), The Constitution of Society: Outline of the Theory of Structure, University of California Press, Berkeley, CA.

Gilbert C. (2001), « Retour d'expérience : théorie et pratique. Le rapport de la Commission d'Enquête britannique sur l'Encéphalopathie Spongiforme Bovine (ESB) au RoyaumeUni entre 1986 et 1996 ", Cahiers du GIS Risques Collectifs et Situations de Crise, Nํ1, juillet.

Girin J. (1990), « L'analyse empirique des situations de gestion : éléments de théorie et de méthode », in Martinet A.-C. (dir.) : Epistémologies et sciences de gestion, Economica, Paris, p. 141-182.

Godé-Sanchez C. (2008), « Les TIC comme leviers du changement : une analyse du cas des Armées américaines en Afghanistan ", Systèmes d'Information et Management, Vol. 13, No1, p. 7-30.

Godé-Sanchez C. (2010a), "Se coordonner en environnement volatil : les pratiques de coordination développées par les pilotes de chasse », Finance, Contrôle, Stratégie, Vol. 


\section{Construire le sens par le retour d'expérience: le cas de l'Equipe de Voltige de l'Armée de l'air}

13, N³, p. 61-93.

Godé-Sanchez C. (2010b), "Leveraging coordination in project-based activities: What can we learn from military teamwork?", Project Management Journal, Special Issue: Project management in extreme situation, Vol. 41, №3, p. 69-78.

Journé B. et Raulet-Crozet N. (2008), « Le concept de situation : contribution à l'analyse de l'activité managériale dans un contexte d'ambiguïté et d'incertitude », M@n@gement, Vol. 11, N¹, p. 27-55.

Klein G., Feltovich P.J., Bradshaw J.M. et Woods D.D. (2004), "Common ground and coordination in joint activity", in Rouse W.R. et Boff K.B. (dir.): Organizational simulation. John Wiley, New York, p. 139-184.

Lièvre P. et Gautier A. (2009), « Les registres de la logistique des situations extrêmes: des expéditions polaires aux services d'incendie et de secours ", Management \& Avenir, Vol. 4, N²4, p. 196-216.

Lièvre P. et Rix-Lièvre G. (2009), « L'observatoire de l'organisant : mode d'interprétation des matériaux qui en sont issus ", Revue Internationale de Psychosociologie, Vol. 15, No35, p. 161-178.

Nizet J.et Pichault F. (2011), "Standards et jeux de pouvoir en situation extrême ", Management \& Avenir - ce numéro.

Pichault F., Bouty I., Drucker-Godard C., Nizet J., Godé-Sanchez C. et Lièvre P. (2010), "Coordination practices in extreme situations: Case studies, analytical framework and methodological stakes", $26^{\text {th }}$ EGOS Colloquium, July 2-4, Lisbon, Portugal.

Picq T. (2005), "Comment développer la performance collective ? Quand le handballeur vient au secours du manager », Gérer et Comprendre, N79, p. 76-84.

Quinn R. et Dutton J. (2005), "Coordination as energy-in-conversation", Academy of Management Review, Vol. 30, №1, p. 36-57.

Rico R., Sanchez-Manzanares M. et Gibson C., (2008), "Team implicit coordination process: A team knowledge-based approach", Academy of Management Review, Vol. 33, No1, p. 163-184.

Ron N., Lipshitz R. et Popper M. (2006), "How organizations learn: Post-flight reviews in an F-16 squadron", Organization Studies, Vol. 27, №8, p. 1069-1089.

Sève C., Bourbousson J., Poizat G., et Saury J. (2009), " Cognition et performance collectives en sport ", Intellectica, Vol. 2, N52, p. 71-95.

Van Maanen J. et Schein E. (1979), "Toward a theory of organizational socialization", Research in Organizational Behavior, Vol. 1, p. 209-264.

Weick K. (1979), The Social Psychology of Organizing, Random House, Second Edition, New-York.

Weick K. (1993), "The collapse of sense-making in organizations: The Man Gulch disaster", Administrative Science Quarterly, Vol. 38, p.628-652.

Weick K. (1995), Sensemaking in Organizations, Sage Publications, London.

Wirtz B., Mathieu A. et Schilke O. (2007), "Strategy in high-velocity environment", Long Range Planning, Vol. 40, p. 293-313.

Yin R. (2003), Case Study Research: Design and Methods, Sage Publication, Applied Social Research Methods Series, Third Edition, London. 


\title{
Emergence de l'agir collectif dans la course à la voile : rythme et coordination
}

\author{
par Isabelle Bouty ${ }^{251}$ et Carole Drucker-Godard ${ }^{252}$
}

Résumé

L'article s'inscrit dans la thématique générale de la coordination des compétences et s'attache à comprendre comment les membres d'une équipe coordonnent leurs contributions, alors qu'ils agissent dans une situation distribuée et dans un environnement changeant. La recherche repose sur l'étude du cas de l'équipage d'un voilier de course. Nos résultats suggèrent que le processus d'émergence de l'agir collectif dans cet environnement est construit par des acteurs clés, centre de production et de diffusion de sens pour l'équipage. Ils mettent enfin en lumière un rythme à deux temps dans la coordination: conduite et opérations.

Abstract

The article investigates coordination between individuals in distributed team in rapidly changing environments. Our analysis is based on the qualitative study of the crew of a racing yacht. We show that coordination emerges on the base of sensemaking by central actors (helmsman, tactician, and bowman). We also show that coordination is fundamentally a rhythmic process with a two-timed tempo: conduct and operations.

Le travail collectif est aujourd'hui une caractéristique organisationnelle forte (Orlikowski 2002, Brown \& Eisenhardt 1997, par exemple). Le cadre théorique sur lequel nous nous fondons est celui de la pratique en situation distribuée (Orlikowski 2002, Hutchins, 1991, Brown \& Eisenhardt 1997). Le travail y est distribué entre des individus spécifiques, qui agissent à dessein et de manière réfléchie, dans le flux d'action dans lequel ils sont pris, ainsi que dans le contexte social et physique de leur activité particulière (Orlikowski, 2002). Nous devons comprendre la performance organisationnelle (au sens d'accomplissement) comme le résultat de l'engagement dynamique des individus, dans un contexte, à un moment et à un endroit particuliers. Elle est le résultat de pratiques contextualisées. Les individus doivent en permanence s'adapter et émettre des jugements afin de se coordonner, dans un processus dynamique dont dépend la performance organisationnelle, ce que certains auteurs ont baptisé la coordination implicite (Rico et al. 2008). 


\section{Emergence de l'agir collectif dans la course à la voile : rythme et coordination}

Quelques premières explorations ont été conduites par le passé mais la plupart analysent des cas où le phénomène a trouvé une issue peu favorable (par exemple, Weick 1993), même si certains travaux font exception (Bigley \& Roberts 2001 ou Rico et al. 2008) en jetant les bases d'une approche plus théorique. Par ailleurs, nombre de ces travaux ont porté sur des organisations hautement fiables, donc très particulières. Leur examen mène à des conclusions dont l'applicabilité aux organisations plus conventionnelles est discutable (Vogus \& Welbourne 2003). Enfin, la coordination n'est pas analysée comme à l'œuvre quotidiennement dans les organisations. La répétition du phénomène n'a en particulier pas été considérée, ce qui limite tout autant la nature des conclusions que leur applicabilité.

Dans le même temps, les évolutions environnementales s'accélèrent pour de nombreuses organisations. Les environnements sont turbulents. Ils rendent la prévision très difficile et nécessitent une adaptation permanente de la part des membres de l'organisation. Le recours à des processus planifiés et standardisés, modes classiques de coordination, devient donc souvent impossible. La plupart des recherches classiques s'inscrit dans une perspective d'environnement relativement stable ou au rythme d'évolution faible, donc prévisible et planifiable (Rico et al. 2008). Elles apportent ainsi des contributions quelque peu limitées au regard des connaissances organisationnelles, dans un contexte où la "coordination implicite» (Rico et al. 2008) devient cruciale. Cette coordination peut être définie comme la coordination par laquelle «les membres d'une équipe anticipent les actions et les besoins de leur collègues et de leurs tâches et ajustent leur comportement de manière dynamique sans avoir besoin de communiquer directement ou de planifier leur activité» (Rico et al 2008: 164). Si cette définition n'est pas parfaite, notamment parce qu'elle assimile rapidement la communication à un processus formel, elle est certainement la plus proche du phénomène qui nous intéresse ici, notamment parce qu'elle s'inscrit en contradiction avec les processus de formalisation et de planification traditionnellement attachés à la coordination. Dans une perspective de recherche, il est donc nécessaire de s'interroger sur ce processus d'ajustement permanent afin de mieux le connaître et le comprendre.

L'article s'articule autour de la problématique de la coordination d'une équipe en situation distribuée et extrême. II se scinde en trois parties. Après avoir décrit dans une première partie les résultats des recherches précédemment menées sur la coordination en environnement changeant, sous l'angle de la cognition, la deuxième partie porte sur l'étude empirique d'un équipage de voilier en situation de régate. Dans ce cas, nous abordons en particulier la situation distribuée dans la mesure où il existe une interdépendance très forte entre acteurs et où les postes, clairement définis et répartis, ne sont pas interchangeables. Même si les membres d'équipage sont capables d'occuper chaque poste sur le bateau, ils n'exerceront jamais deux rôles simultanément pendant la course. La troisième 
partie tire les enseignements issus de l'étude empirique, mettant en avant le rôle central de trois acteurs clés dans la production et la diffusion de sens pour l'ensemble de l'équipage. Ces mêmes acteurs font émerger un rythme de l'agir collectif.

\section{Coordination et agir collectif}

Dans la littérature en management, plusieurs courants ont indirectement abordé la question de la coordination en environnement changeant sans toutefois la traiter directement. En particulier les travaux portant sur les organisations hautement fiables, ceux sur le management de crise et l'improvisation présentent un grand intérêt. Bien qu'à chaque fois les domaines ne recouvrent pas exactement la situation qui nous intéresse, les travaux passés ont mis en évidence certains éléments significatifs pour notre étude, c'est pourquoi nous les exposons ici dans une perspective transversale.

Les travaux passés concourent à souligner le rôle des recouvrements cognitifs entre membres d'un groupe pour la mise en cohérence des jugements et des actions. Dans la littérature, ces recouvrements apparaissent de plusieurs ordres et sous diverses appellations. Cannon-Bowers \& Salas (2001) suggèrent de les classifier en quatre grandes catégories:

- Le savoir spécifique à la tâche, qui concerne essentiellement la connaissance des actions, procédures et séquences nécessaires à la réalisation d'une tâche. Les termes «knowledge redundancy» ou «knowledge overlap» (Hutchins 1990, 1995) couvrent la même notion.

- Le savoir relatif à la tâche, qui porte sur l'articulation d'un ensemble de tâches. Si ces savoirs individuels se recouvrent plutôt que d'être mutuellement exclusifs, les systèmes peuvent produire une action robuste et flexible (Bigley \& Roberts 2001, Rico et al. 2008, Zack 2000, Weick 1998, Moorman \& Miner 1998).

- La connaissance des co-équipiers qui concerne la connaissance et la compréhension des autres : savoir qui fait quoi dans le groupe plus que comment accomplir chaque tâche (Zack 2000, Kamoche \& Cunha 2001, Weick 1993).

- Les attitudes et les croyances, qui concernent des modèles sous-jacents; les schémas mentaux partagés (normes, standards, concepts) aident au travail collectif.

La littérature apporte une deuxième conclusion importante : pour intégrer le changement, les individus essaient d'agir de manière sensée et créative compte tenu du sens qu'ils donnent à la situation collective qu'ils sont en train vivre. Le parallèle est souvent fait entre l'improvisation en jazz et une conversation qui fait émerger le sens et/de la production au fur et à mesure que l'action se déploie (Weick 1998, Zack 2000). La connaissance de la tâche à accomplir est plus importante que le seul respect des procédures (Hirschhorn 1993). Weick 


\section{Emergence de l'agir collectif dans la course à la voile : rythme et coordination}

(1993) insiste sur cette notion de sensemaking pour souligner que les acteurs essaient d'agir d'une manière qui leur semble rationnelle. Lorsque les individus ne trouvent plus de sens à ce qu'ils vivent et ne peuvent plus le reconstruire, la situation devient rapidement catastrophique (Weick 1993) parce que l'action intelligente n'est plus possible (Weick \& Roberts 1993).

Les travaux passés insistent aussi sur l'importance de l'existence d'une forme de structure dans le groupe. Plus précisément, ils s'accordent à montrer qu'en l'absence de structure, toute la coordination repose exclusivement sur le processus interactif, l'exposant ainsi aux erreurs de compréhension, aux mauvaises interprétations et aux défaillances de communication. Si l'étude d'une unité de pompiers (Bigley \& Roberts 2001) montre que des formes de structures bureaucratiques peuvent exister et demeurer fiables, à la seule condition qu'elles soient flexibles, la littérature souligne de manière récurrente les problèmes liés à la structure centralisée classique (Hatch 1999) notamment dans les environnements turbulents (Volderba 1996). Par opposition, la notion de structure minimale est la plus frappante dans la littérature sur l'improvisation (Barret 1998, Hatch 1999, Zack 2000, Kamoche \& Cunha 2001). II s'agit d'une structure principalement sociale. Par son existence, elle sert de base à la compréhension de la situation alors qu'elle se déploie. Par son minimalisme, elle permet la créativité. Les travaux passés s'accordent finalement à souligner le processus de structuration permanent plus que la structure elle-même (Weick 1993, Hatch 1999), y compris pour les situations dans lesquelles la structure est de nature plus formelle (Bigley \& Roberts 2001).

La quatrième conclusion issue des travaux passés porte sur le rôle de la communication verbale et non verbale. Ainsi que le soulignent Bigley \& Roberts (2001), Eisenhardt (1993) ou Bastien et Hostager (1988), la communication facilite la convergence des représentations opérationnelles qui servent de base à la construction de l'action. De plus et ainsi que le soulignent Quinn \& Dutton (2005), communication et coordination sont extrêmement proches l'une de l'autre, même souvent comprises comme synonymes. L'interaction implique une large part d'écoute et de communication (Barrett 1998, Bastien \& Hostager 1998, 1992, Hatch 1999). Finalement et ainsi que le souligne Crowston (1991), la coordination est en large partie un processus d'échange d'informations. Mais la communication reste un processus parfois fragile : la pression imposée aux acteurs par la situation et les conditions extérieures peut rendre la communication aussi difficile qu'elle est importante (Weick 1993).

La littérature est finalement riche de conclusions sur certaines sources possibles de coordination mais elle ne décrit pas le phénomène en pratique et nous laisse donc avec une compréhension très réduite de celui-ci.

Fort de ces résultats et de ces lacunes, nous avons mené une étude empirique afin d'analyser de manière approfondie comment naît l'agir collectif dans les 
organisations distribuées, agissant dans une situation évolutive, risquée et incertaine, comment les acteurs coordonnent en pratique leurs contributions individuelles respectives pour former ensemble l'agir collectif.

\section{L'agir collectif au sein d'un équipage de voilier : exemple de coordination en situation changeante}

\section{Contexte de la recherche}

Notre étude de cas porte sur une équipe engagée dans une activité distribuée (un membre d'équipage n'occupe qu'un poste), conduite dans un environnement hautement variable et où les individus doivent donc en permanence coordonner leurs contributions de manière renouvelée : l'équipage d'un voilier en situation de régate.

Le travail empirique qui fonde cette recherche est qualitatif, basé sur une étude de cas. La voile en équipe s'est vite imposée comme un terrain particulièrement pertinent : l'équipage est composé d'individus qui agissent de manière coordonnée pour concourir à la formation de l'output collectif (la navigation du bateau). En même temps, les facteurs de variabilité environnementaux sont très nombreux et pour certains, puissants. Tout va vite et il faut en permanence faire face à des conditions nouvelles.

A titre d'illustration, les principaux changements environnementaux survenant en régate sont indiqués dans le Tableau 1 :

Tableau 1: Principaux changements environnementaux en régate

\begin{tabular}{|c|c|}
\hline & Principaux changement possibles \\
\hline $\begin{array}{l}\text { Courants et } \\
\text { vagues }\end{array}$ & $\begin{array}{l}\text { Leur intensité peut croître ou diminuer rapidement selon le temps. } \\
\text { Changent de côté lors des virements de bord et empannages. }\end{array}$ \\
\hline Vent & $\begin{array}{l}\text { Peut changer de direction, se renforcer ou se calmer. } \\
\text { Change de côté lors des virements de bord et empannages }\end{array}$ \\
\hline Bruit & Son intensité est variable selon la météo, la vitesse et les mouvements à bord. \\
\hline Concurrents & $\begin{array}{l}\text { Changent souvent de position et de vitesse en fonction de leur stratégie. } \\
\text { Peuvent être très proches et / ou en position de requérir la priorité. }\end{array}$ \\
\hline Visibilité & $\begin{array}{l}\text { Varie selon la météo et les vagues. } \\
\text { Le soleil et les vagues changent de côté lors des virements de bord et } \\
\text { empannages. }\end{array}$ \\
\hline Matériel & $\begin{array}{l}\text { Peut subir des avaries parfois irrémédiables (accidents humains compris par ex. } \\
\text { lorsque la baume change violemment de côté lors des empannages) } \\
\text { Les voiles et le bateau doivent être réglés en permanence pour être ajustés aux } \\
\text { conditions de vent. }\end{array}$ \\
\hline
\end{tabular}




\section{Emergence de l'agir collectif dans la course à la voile : rythme et coordination}

Le cas que nous avons étudié est celui d'un équipage en régate sur un voilier monocoque pour 7 équipiers et engagé dans le Tour de France à la Voile (TFV). La figure ci-dessous offre une représentation schématique des positions des équipiers à bord :

Figure 1: Positions des équipiers à bord

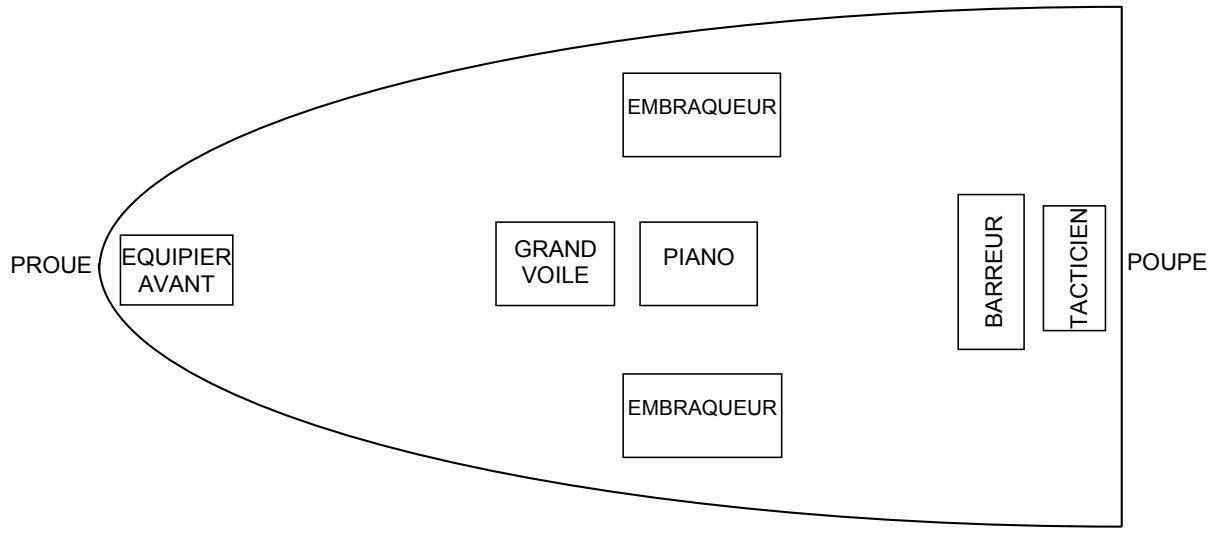

A bord, le barreur donne le cap au bateau à l'aide de la barre et en tenant compte de la stratégie élaborée par le tacticien qui, lui, dispose sur ses quadrants des informations de vitesse et de météo. Les autres équipiers concourent au réglage du bateau pour atteindre la meilleure vitesse. Ils règlent les voiles, la tension des divers cordages, le gîte. Un voilier étant propulsé par l'action combinée du vent et du courant, la navigation en équipage est un agir collectif dans lequel chaque équipier tient une place fondamentale car les réglages sont nombreux, mutuellement dépendants et en relation avec le cap et les éléments environnementaux. Qu'un réglage soit négligé et les conséquences sont immédiates : au mieux la vitesse du bateau décroit, au pire le bateau peut chavirer ou un équipier être blessé. La navigation à la voile est aussi un exercice dans lequel les sensations de chaque équipier sont très importantes pour ces réglages. S'il existe moult écrits et discours sur la «théorie» de la navigation, c'est à la pression sur la barre que le barreur sent le cap et les relâchements éventuels à donner, c'est entre leurs mains que les équipiers sentent la tension des cordages, à la forme et au bruit qu'ils jugent du réglage des voiles. Rappelons que n'importe quel équipier est capable d'occuper chaque poste, mais que pendant la course ils sont chacun assigné à un unique poste ; ainsi les rôles sont mutuellement exclusifs.

L'équipage particulier que nous avons choisi d'observer venait d'être en partie renouvelé lorsque nous débutions l'étude. Le skipper était bien connu dans le monde de la voile et avait déjà gagné à plusieurs reprises le TFV. Les nouveaux étaient quant à eux plus jeunes et ne se connaissaient pas tous, mais avaient 
acquis une expérience de la voile de compétition dans d'autres contextes. Nous pouvions ainsi observer certains recouvrements cognitifs dans cet équipage (tous les équipiers étaient des marins à part entière), mais il leur fallait encore apprendre à «régater ensemble». Cette situation nous offrait l'opportunité d'observer la mise en place de la coordination à bord au quotidien et cela pendant l'intégralité du TFV.

\section{Unité de recherche : la régate}

Une régate est un ensemble de manœuvres réalisées dans un temps et un espace limités. En régate, le voilier effectue un parcours «ponctué» de bouées, dans le respect de règles strictes. Pour bénéficier au mieux du vent, le voilier doit adopter une trajectoire en «zigzag» le long du parcours et donc effectuer de nombreuses manœuvres. La figure 2 illustre cette trajectoire :

Figure 2 : Manœuvres de progression sous le vent et virement de bord

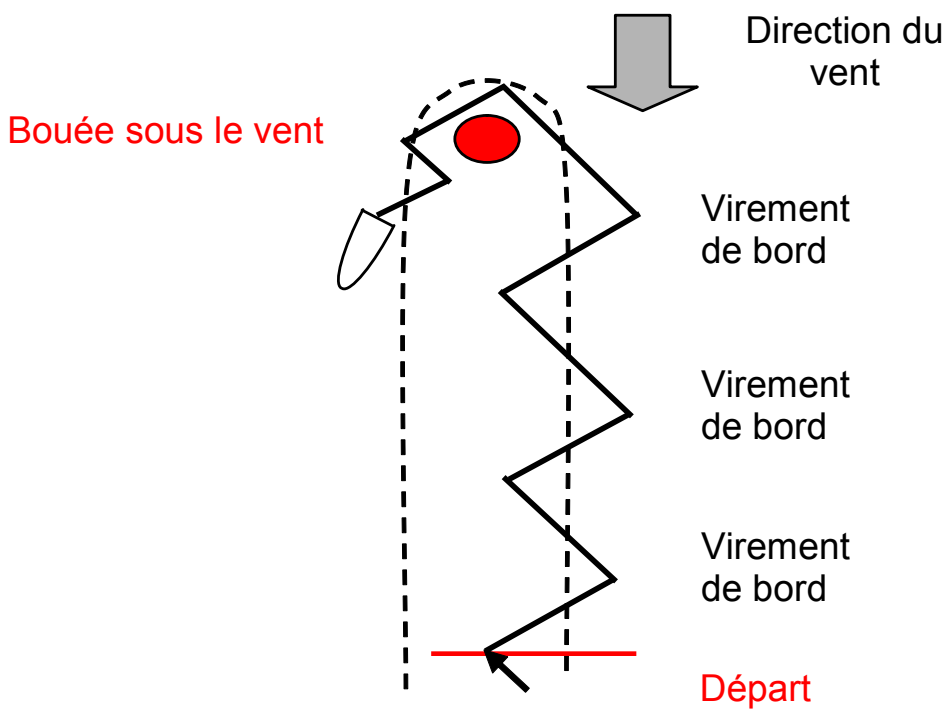

Cette figure simplifiée représente une trajectoire théorique, ne prenant en compte ni les changements de météo ni les autres éléments pouvant perturber la trajectoire initiale. En particulier, le nombre souvent élevé des concurrents génère des changements environnementaux importants et très inattendus. De ce fait le passage autour de la bouée sous le vent est un moment réellement clef de la course où de nombreuses places peuvent être gagnées ou perdues. Finalement, la situation réelle ressemblerait plus exactement au schéma représenté en Figure 3 : 


\section{Emergence de l'agir collectif dans la course à la voile : rythme et coordination}

Figure 3 : Traçage de la flotte en progression sous le vent et virement de bord

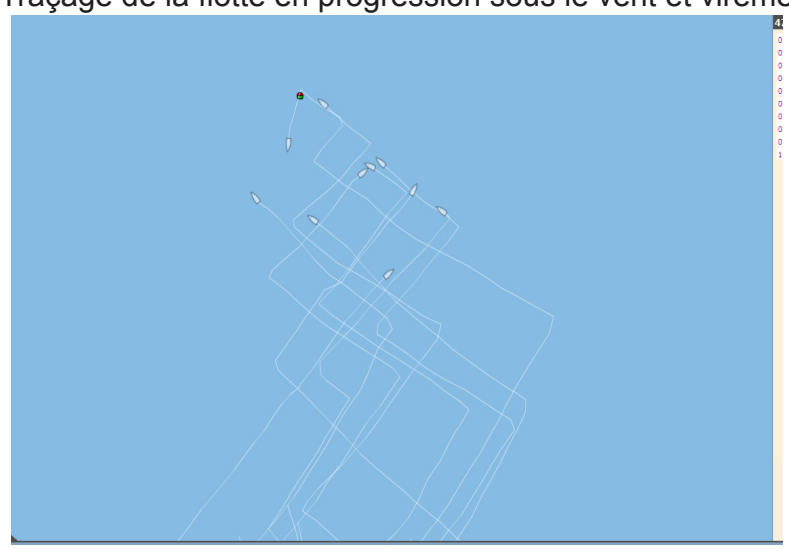

Ainsi que l'illustre le schéma, une régate nécessite de nombreuses manœuvres pour intégrer les changements tout au long de la course. Nous avons donc ici l'opportunité de considérer la pratique de coordination répétée dans la formation de l'agir collectif.

\section{Méthodologie}

La collecte des données pour les régates a pris plusieurs formes: observations, enregistrements à bord, entretiens.

L'observation a eu lieu depuis le bateau du comité d'organisation. Depuis cette position, l'observation de l'enchainement des manœuvres était possible dans le respect du règlement de course. Pour compléter l'observation et recueillir des données en temps et situation réels, nous avons enregistré (magnétophone et micro-cravate étanches) tous les équipiers à bord durant trois étapes du TFV. Ces 21 enregistrements ont ensuite été retranscrits mais nous insistons sur la difficulté du travail de retranscription due au bruit du vent, de la mer, du matériel... et au dispositif mis en place pour entendre simultanément les cassettes des 7 membres d'équipage. Nous n'avons pas pu, pour des raisons pratiques, repasser les enregistrements avec l'ensemble de l'équipage. Nous avons cependant fait un débriefing régulier des enregistrements avec le skipper et de manière plus ponctuelle avec quelques équipiers. Les données recueillies à bord ont été complétées par des entretiens. Nous avons interviewé chaque membre naviguant de l'équipage, avant chacune des trois régates. L'équipage variant suivant les régates, nous avons mené en tout 21 entretiens avec 11 équipiers différents. Ces entretiens ont été enregistrés, intégralement retranscrits et codés. Enfin, nous avons assisté aux réunions de briefing et de débriefing de l'équipage, avant et à l'issue des trois régates considérées. Outre ces données très riches, nous avons recueilli des données plus informelles en discutant avec des équipiers restés à terre pendant les régates. 


\section{Résultats de terrain : La coordination pour la formation de l'agir collectif}

\section{Des acteurs clés}

L'analyse des régates a mis en lumière l'existence d'un sous-groupe d'acteurs clés au sein de l'équipage, notamment sur la base du traçage des flux de communication à bord durant la régate, et qui sont schématisés dans la figure 4:

Figure 4 : Flux de communication à bord

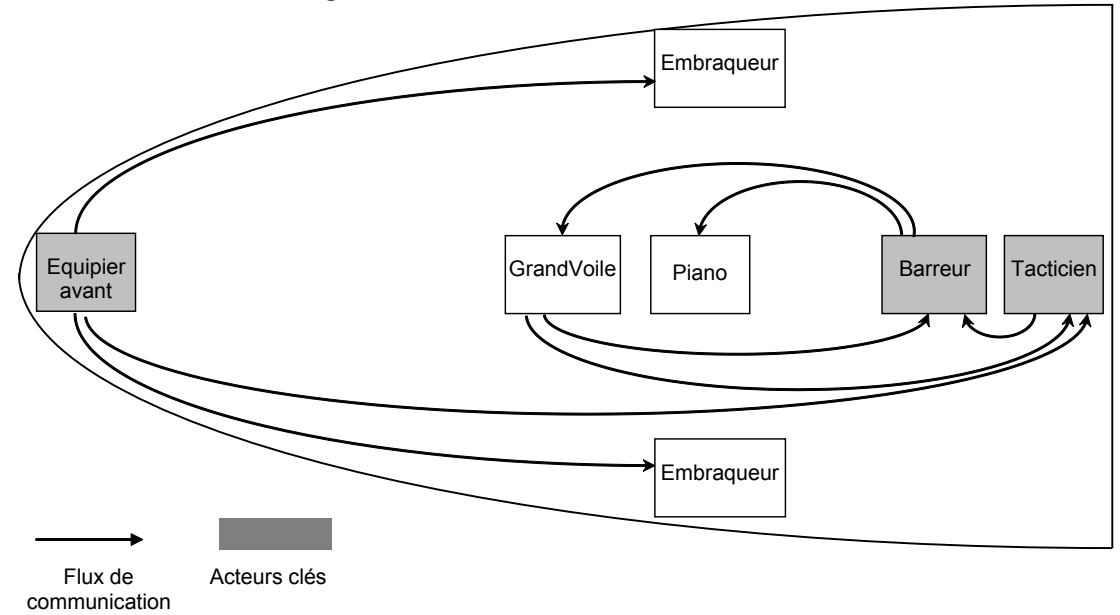

Comme le montre la Figure 4, tous les acteurs prennent part au processus de communication à bord, ne serait-ce que parce qu'ils sont récepteurs des informations ou des annonces. Mais il apparaît clairement que l'essentiel de la communication se passe entre trois acteurs principaux : le barreur, le tacticien et l'équipier avant. Notre analyse révèle que cette sous-équipe d'acteurs clés est le centre de construction et de diffusion du sens au sein de l'équipage pour le développement de l'action collective.

En premier lieu, ils sont le centre de la production de sens. Ce sont les choix tactiques (traduits en annonces) qui orientent l'action de l'équipage. Ces choix sont élaborés en réponse à une vision de la situation vécue par l'équipage. Ils sont donc le reflet d'un sens donné à cette situation. Or la tactique est une construction de ces trois acteurs clés. Elle reflète donc le sens construit par l'équipier avant, le tacticien et le barreur. Ils sont ainsi le centre de production de sens pour l'équipage entier. Bien sûr, ils produisent un sens, leur sens de la situation ; il existe certainement d'autres manières de comprendre des événements donnés. D'ailleurs tous les concurrents d'une régate ne font pas 


\section{Emergence de l'agir collectif dans la course à la voile : rythme et coordination}

les mêmes choix tactiques, témoignant ainsi de la diversité des sens possibles. Mais, en même temps, la sous-équipe d'acteurs clés n'offre pas le choix du sens à l'équipage ; c'est elle qui articule les informations et ainsi élabore l'interprétation de la situation sous-jacente aux annonces faites à l'équipage.

En deuxième lieu et pour renforcer la prédominance du sens qu'ils construisent, les trois acteurs clés disposent d'un outil de diffusion : la communication. Elle est le moyen privilégié de diffusion du sens auprès de l'équipage. Le sens est tout d'abord diffusé à travers les annonces qui déclenchent l'action. Mais il est aussi porté lors des échanges d'informations et notamment les mises en garde ou les appréciations, plus fréquentes lors des phases de conduite. Par exemple : "Attention on va renvoyer très vite les gars» ou "on a plein de vagues là» (enregistrements).

Ainsi, l'importance de la communication à bord est liée à la nécessité de construire l'action sur la base d'un sens collectif partagé. Ce sens est produit et diffusé par les acteurs clés. C'est la raison pour laquelle cette structure minimale tient une place si importante dans la communication à bord. Le développement coordonné des actions individuelles des équipiers est fondé sur le sens produit et diffusé par ces acteurs en particulier.

\section{La coordination comme un rythme}

L'observation de régates a permis de mettre en lumière le rythme à deux temps de la pratique de la coordination, rythme guidé par les trois acteurs clés. Nos enregistrements laissent très clairement entendre des changements de rythme dans la communication et l'action sur le bateau. Certains moments sont intenses : discours, échanges, bruit. D'autres moments sont calmes et plus détendus. L'analyse de nos données nous a permis d'identifier ces rythmes comme deux facettes de la coordination : les opérations et la conduite.

\section{Les opérations}

Les phases d'opération sont caractérisées par leur grande intensité, tant dans la communication, l'action, le contexte que dans la tension perceptible au sein de l'équipage. On entend tout d'abord de nombreux échanges verbaux porteurs d'information. Par exemple :

«t'as vu la bouée?», ou «le temps, les temps!!?» (enregistrements)

Les questions sont brèves, l'intensité est forte, les réponses courtes. Puis, on entend le barreur annoncer à l'équipage :

«maintenant» (enregistrements)

Immédiatement après s'élève le bruit des cordages et des pièces métalliques qui s'entrechoquent. Puis en quelques secondes le calme revient. L'analyse des notes d'observation, des dialogues et des bruits environnants nous indique que ces moments correspondent à des périodes de changement sur le bateau, 
essentiellement à des manœuvres (telles que décrites plus haut) ou à des réactions à des changements pressentis, tels la remontée d'un concurrent. Les dialogues suivent un schéma récurrent : demandes répétées d'information puis une annonce. Cette annonce est ensuite systématiquement suivie d'une action à bord (observable directement mais aussi identifiable aux bruits du matériel). L'annonce est un véritable déclencheur d'action. Mais il est aussi notable qu'elle prend toujours la forme d'un simple mot (par exemple «maintenant!») ou d'un geste ; elle indique seulement un moment. Enfin, elle est adressée à l'équipage dans son ensemble, non pas à des équipiers spécifiques. Finalement, nous analysons l'annonce comme un déclencheur d'action rythmant les contributions des équipiers.

\section{La conduite}

Les phases de conduite sont caractérisées par leur moindre intensité. Ce sont de longs moments de calme durant lesquels la communication est moins intense et essentiellement porteuse d'informations. Les échanges sont beaucoup moins nombreux. La pression semble moins forte. Pourtant ce sont des périodes durant lesquelles on entend le matériel cliqueter, les cordages glisser, les équipiers bouger (enregistrements et observations). Ces bruits témoignent d'une activité continue sur le bateau mais dont les ressorts de coordination sont certainement différents. N'ayant pas pu systématiquement collecter des données visuelles directes sur l'activité du bateau durant ces moments, nous ne pouvons conjecturer que sur la base de nos observations depuis le bateau du comité. II nous apparaît que durant les moments de calme, une activité se poursuit sur le bateau. Les équipiers naviguent ensemble sans avoir besoin d'échanger des informations. Ils fondent leur coordination sur leur connaissance de la navigation (recouvrements cognitifs) appliquée à des situations momentanément plus stables. Nous comprenons aussi ces périodes de conduite comme intimement liées aux opérations. En effet, d'une part, les équipiers conduisent le bateau vers un nouveau lieu d'opération comme une bouée ou un point de virement. D'autre part ils sont à l'écoute du bateau et de l'environnement, cherchant à détecter tous les indices d'un changement, afin de préparer la nouvelle phase d'opération. D'ailleurs, nous entendons des dialogues tels:

Barreur : " la vitesse c'est bien ?» Tacticien : "c'est bon, on reprend » (enregistrement)

Barreur : " on dirait [que le concurrent Y] perd énorme à la bouée » (enregistrement)

Barreur : "OK?, OK réglages, OK le temps, on est d'accord les gars ?»

Ainsi, le rythme sert à se coordonner. Opérations et conduite sont donc les deux temps indissociables du processus d'émergence de l'agir collectif en situation distribuée et turbulente. 


\section{Emergence de l'agir collectif dans la course à la voile : rythme et coordination}

\section{Discussion et conclusion}

Les organisations sont aujourd'hui fréquemment décrites comme distribuées. Parallèlement, elles évoluent dans des environnements de plus en plus évolutifs, incertains et risqués. Dans ces conditions, la performance peut être comprise comme une réalisation collective fondée sur la coordination des contributions individuelles des membres de l'organisation. Mais les travaux passés décrivent ce phénomène de manière très partielle et indirecte. C'est la raison pour laquelle nous avons exploré le processus d'émergence de l'agir collectif en situation distribuée et extrême. Nous avons pour cela mené une étude empirique auprès de l'équipage d'un voilier de course. Nos conclusions font apparaître d'une part que la coordination est fondée sur un nombre réduit d'acteurs clés producteurs de sens pour l'équipage et d'autre part qu'elle est un processus à deux temps.

Il existe dans l'équipage une sous-équipe d'acteurs clés, centre de production et de diffusion de sens pour l'ensemble de l'équipage. De par leur rôle, ces acteurs clés sont la principale source de mise en cohérence des actions individuelles. Nous rejoignons ici Weick (1993) dans sa vision des relations d'équilibrage entre sens et structure.

Nous montrons aussi que la coordination en situation distribuée et changeante est une pratique à deux temps : conduite et opérations. Les phases de conduite correspondent à des moments de relative stabilité environnementale et de veille. Les phases d'opération sont quant à elles des moments de développement de l'action. Dans ces deux phases (et de manière plus marquée lors des opérations) l'agir collectif émerge sur la base du sens produit et diffusé par les acteurs clés, tout particulièrement à travers les annonces tactiques de manœuvre. Notre analyse rejoint sur certains points celle de Brown \& Eisenhardt (1997) qui insistent aussi sur la notion de rythme comme source de succès. Nos conclusions représentent cependant une avancée par rapport à celles de ces auteurs car Brown \& Eisenhardt font référence à un rythme calendaire ou du moins largement pré-défini dans l'organisation, fruit d'une vision fondamentalement ancrée dans la séparation traditionnelle en stratégie, entre décision et action. En revanche, l'alternance opération/conduite que nous mettons en lumière n'est pas programmée et dépend intégralement des variations environnementales. Elle n'est pas non plus fondée sur une opposition décision/action, elle est la coordination. Finalement, l'alternance opération/conduite est l'intégration coordonnée des variations environnementales.

Notre recherche apporte deux contributions théoriques principales. En premier lieu, nous avons analysé un phénomène qui n'avait jamais fait l'objet d'une étude directe auparavant. Nous avons exploré la coordination en situation distribuée et incertaine alors que les travaux passés se sont au mieux concentrés sur une de ces deux perspectives, avec une préférence marquée pour l'analyse 
de la coordination dans des situations distribuées en environnement stable. Deuxièmement, nous avons étudié ce phénomène dans le temps, de manière dynamique, alors que les travaux passés ont analysé des évènements ponctuels. Nous mettons donc en lumière la pratique de la coordination alors que les travaux passés se sont focalisés sur ses sources. En effet, certaines de nos conclusions, notamment celles relatives à la description du rythme de la coordination, n'ont pu émerger que par le biais d'une observation répétée du phénomène.

Enfin, notre recherche est de nature exploratoire et elle offre des conclusions qui restent à tester dans une perspective de vérification. Elle ouvre ainsi des voies de recherche futures. Mais compte tenu de la complexité du processus étudié et de la difficulté de collecte des données, il s'agit là d'un travail difficile et de longue haleine.

\section{Références}

Barrett, FJ. 1998. Creativity and improvisation in jazz and organizations: implications for organizational learning. Organization Science. 9(5) 605-623.

Bastien, DT., TJ. Hostager. 1988. Jazz as a process of organizational innovation. Communication Research. 15(5) 582-602.

Bastien, DT., TJ. Hostager. 1992. Cooperation as communicative accomplishment: a symbolic interaction analysis of an improvised jazz concert. Communication Studies. 43 (summer) 92-104.

Bigley, G.A., KH. Roberts. 2001. The incident command system: High-reliability organizing for complex and volatile task environments. Academy of Management Journal. 44(6) 1281-1299.

Bourgeois, L.J., K.M. Eisenhardt. 1988. Strategic decision processes in high velocity environment: Four cases in the microcomputer industry. Management Science. 34(7) 816835.

Brown, S., KM. Eisenhardt. 1997. The art of continuous change: linking complexity theory and time-paced evolution in relentlessly shifting organizations. Administrative Science Quarterly. 42(1) 1-34.

Cannon-Bowers, J.A., E. Salas. 2001. Reflexions on shared cognition. Journal of Organization Behaviour. 22 195-202.

Crowston, K. 1991. Towards a coordination cookbook. Recipes for multi-agent action. Unpublished Doctoral Dissertation, MIT Sloan School of Management; Boston, MA.

Eisenhardt, K.M. 1993. High reliability organizations meet high velocity environments: common dilemmas in nuclear power plants, aircraft carriers and microcomputer firm. K.H. Roberts, ed. New challenges to understanding organizations. Macmillan, New York., 117153.

Hatch, M.J. 1999. Exploring the Empty Spaces of Organizing: How Improvisational Jazz Helps Redescribe Organizational Structure. Organization Studies 21(1) 75-100.

Hirschhorn, L.1993. Hierarchy versus bureaucracy: the case of a nuclear reactor. KH. Roberts, ed. New challenges to understanding organizations. Macmillan, New York, 137149.

Hutchins, E. 1990. The technology of team navigation. J. Galagher, R.E. Kraut, C. Egido, eds. Intellectual teamwork: social and technological foundations of cooperative work. Lawrence Erlbaum Associates, Hillsdale, 191-221.

Hutchins, E. 1991. Organizing work by adaptation. Organization Science. 2(1) 14-39. 


\section{Emergence de l'agir collectif dans la course à la voile : rythme et coordination}

Hutchins, E. 1995. Cognition in the wild. MIT Press, Cambridge Mass.

Kamoche, K., M.P. e Cunha. 2001. Minimal structures: from jazz improvisation to product innovation. Organization Studies. 22(5) 733-764.

Moorman, C., AS. Miner. 1998. Organizational improvisation and organizational memory. Academy of Management Review. 23(4) 698-723.

Orlikowski, WJ. 2002. Knowing in practice: Enacting a collective capability in distributed organizing. Organization Science. 13(3) 249-273.

Quinn, WR., JE. Dutton. 2005. Coordination as energy-in-conversation. Academy of Management Review. 30(1) 36-57.

Rico, R N, Sanchez-Manzanares M, Gil F, Gibson C. 2008. Team implicit coordination processes: a team knowledge-based approach. Academy of Management Review, 33(1): 163-184.

Vogus, TJ., TM. Welbourne. 2003. Structuring for high-reliability: high reliability practices and mindful processes in reliability-seeking organizations. Journal of Organizational Behaviour. 24(7) 877-903.

Volderba, HW. 1996. Toward the flexible form: how to remain vital in hypercompetitive environments. Organization Science. 7(4) 359-374.

Weick, KE. 1993. The collapse of sensemaking in organizations: The Mann Gulch disaster. Administrative Science Quarterly. 38(4) 628-652.

Weick, KE. 1998. Introductory essay. Improvisation as a mindset for organizational analysis. Organization Science. 9(5) 543-555.

Weick, KE., KH. Roberts. 1993. Collective mind in organizations: Heedful interrelating on flight decks. Administrative Science Quarterly. 38(3) 357-381.

Zack, MH. 2000. Jazz improvisation and organizing: once more from the top. Organization Science. 11(2) 227-235. 


\title{
Pratiques de coordination d'un collectif informel en situation extrême : une étude de cas « ancrée » au Groenland
}

\author{
par Pascal Lièvre ${ }^{253}$ et Géraldine Rix-Lièvre ${ }^{254}$
}

Résumé

Abstract

Dans le cadre de la théorie ancrée, l'objet de cette contribution est de rendre compte comment un collectif informel en situation extrême tel une expédition polaire, sans standard organisationnel partagé, s'y prend pour se coordonner. II apparait que le chef d'expédition surdétermine les modes de coordination tout au long du projet. C'est à partir de son expérience en tant que chef d'expédition que celui-ci a construit une manière " invariante » de conduire une expédition qui l'amène à prendre en charge la conception de l'expédition, à coordonner d'une manière classique mais relativement autoritaire la phase de préparation, et à tout faire pour maintenir sa légitimité en tant que chef d'expédition dans la phase de réalisation où l'adaptation doit prévaloir à toute planification.

In the framework of grounded theory, our purpose is to describe how an informal group, like a polar expedition team, without any shared standards, coordinates itself in an extreme situation. The coordination modes are mostly determined by the expedition leader all along the project. From his own practices as an expedition leader, he had constructed a constant way of managing an expedition. He plans and conceives himself the expedition project. During the preparation phase, he coordinates classically the team, even if he is a bit authoritarian. During the raid, wherein adaptation should predominate to the plans, his key concern is to maintain his legitimacy.

La littérature sur la question des modes de coordination en situation volatile, en environnement turbulent ce que nous qualifions plus précisément de situations extrêmes de gestion (Lièvre, Gautier, 2009 ; Pichaut et alii, 2010), fait état de controverses comme en rendent compte dans ce cahier l'article de Nizet et Pichault (2011). Un certain nombre de travaux (Bouty, Godard, 2009 ; GodéSanchez, 2008, 2010a, 2010b ; Pichault, 2002 ; Alsène et Pichault 2007 ; Pichaut et alii, 2010) interpellent des positions théoriques défendues par les théories de la contingence (Lawrence et Lorsch, 1967 ; Thompson, 1967 ; Van de Ven et

\footnotetext{
253. PASCAL LiėVRe, Maître de Conférences HDR, Clermont Université \& Groupe ESC Clermont, CRCGM EA 3849, pascallievre@orange.fr

254. Géraldine Rix-LıĖVRE, Maître de Conférences, Université Blaise-Pascal, Clermont Université, PAEDI EA 4281, geraldine.rix@univ-bpclermont.fr
} 


\section{Pratiques de coordination d'un collectif informel en situation extrême : une étude de cas « ancrée » au Groenland}

al., 1976 ; Mintzberg, 1978 ; Keller, 1994 ; Gupta et al., 1994) selon lesquelles les organisations confrontées à une forte incertitude environnementale doivent délaisser les mécanismes formels de coordination au profit de mécanismes beaucoup plus souples, plus informelles. Pour Pichault et alii (2010), cette posture théorique n'apparaît pas pleinement satisfaisante pour au moins deux raisons. D'une part, elle repose sur une vision essentiellement structurelle de la coordination alors que la coordination a une dimension processuelle importante. D'autre part, le niveau de granularité retenu par les théories de la contingence se situe à l'échelon de l'organisation ou de l'unité de travail et non à l'intérieur de ces dernières. Ainsi, il ne permet pas d'étudier les modes effectifs de coordination située au niveau des interactions individuelles et d'ouvrir " ces boites noires relativement fermées "(Alsène et Pichault, 2007, p. 65) que représentent aujourd'hui encore les modes de coordination. Pour sortir de ces situations d'indécidabilité, il s'agit selon ces auteurs d'ouvrir la boite noire de la coordination en s'intéressant délibérément aux pratiques des acteurs en situation.

C'est ce que nous proposons de faire, dans cet article, en prenant d'une part comme objet de recherche un collectif informel en situation de projet dans un environnement extrême, à savoir une expédition polaire et en investissant d'autre part les pratiques des acteurs en situation dans la perspective d'une épistémologie de la pratique (James, 1909 ; Glaser et Strauss, 1967 ; Bourdieu, 1977 ; Piaget, 1974 ; Schön, 1983 ; Lave et Wenger, 1991 ; Cook et Brown, 1999) en mobilisant un dispositif méthodologique qui se veut délibérément centré sur les pratiques et non sur les discours sur les pratiques (Lièvre, Rix-Lièvre, 2009).

Notre investigation s'inscrit résolument dans le champ de la théorie ancrée de Glaser et Strauss (1967). Renouant avec l'approche pragmatique de James (1909), telle qu'elle est évoquée par Pierre Paillé (2010, p34) dans la préface à la traduction française de «La découverte de la théorie ancrée » (1967/2010), nous construisons un cadre de référence d'une part à même d'intégrer les expériences vécues des acteurs au sens de James (1912) et d'autre part positionnant les discours en référence avec l'action au sens de Ricoeur (1977). C'est sur une démarche abductive, au sens du philosophe pragmatique Pierce, qui traduit selon Richardson (2006) le mieux la démarche initiée par Glaser et Strauss en 1967, que nous nous appuyons pour dégager dans un premier temps les " théories en acte » mises en œuvre par les acteurs en situation, puis de confronter ces " théories substantives " aux théories « formelles » sur la coordination. Nous visons le champ des théories intermédiaires au sens de David (2000) c'est-àdire un registre qui se situe entre les faits mis en forme et le champ des théories universalistes des sciences de gestion. Une grande attention sera portée aux modalités de construction et d'écriture de la situation concrète qui relève du registre de la description au sens de Latour $(2006, p 199)$ : un registre où la quête du détail est essentielle et où le fait de vouloir ajouter une explication à la description est la preuve que la description n'est pas complète et nécessite 
alors de rajouter dans la description le facteur explicatif. Par ailleurs, la nature des inférences entre les différents registres fera l'objet d'un soin tout particulier pour respecter les rationalités endogènes à l'action collective, propre à une épistémologie de la pratique, en mobilisant l'analyse comparative systématique de Glaser et Strauss.

Nous prenons comme objet de recherche, un collectif informel d'acteurs " amateurs ». Ils n'appartiennent à aucune organisation ou institution commune. Ils n'ont jamais fonctionné ensemble dans une situation similaire et donc n'ont pas de routine organisationnelle pré-existante. Ils ne peuvent pas se reposer sur un standard formel codifié de coordination appris collectivement dans une école ou faisant l'objet d'une norme dans une organisation, et enfin ils sont immergés dans un milieu extrême : le milieu polaire. Ainsi, nous nous positionnons dans un contexte de coordination en matière de standard qui est ici orthogonal à des domaines comme le militaire (Gode-Sanchez 2009) ou par exemple la sécurité civile (Bigley, Roberts, 2001 ; Lièvre, Gautier, 2009 ; Gautier, 2010) où ceuxci sont existants, formalisés, codifiés, enseignés, planifiés, exécutés... Nous interrogeons les modes de coordination qui se construisent en situation dans un tel contexte où les standards n'existent pas.

Nous travaillons sur une étude de cas extrême au sens de Yin (2003) à savoir une expédition polaire qui s'est déroulée au Groenland en 2005 dont l'objet est d'entreprendre une traversée à ski en autonomie entre deux lieux, séparés de $800 \mathrm{~km}$. Cette liaison n'avait jamais été réalisée auparavant mais ne constitue pour autant un exploit sportif. Le chef d'expédition, Joël est un expert reconnu dans le milieu. II a réalisé une trentaine d'expédition et a écrit des ouvrages de référence sur la question. Sur le plan méthodologique, les données sur cette expédition ont été recueillies de manière concomitante au déroulement de l'expédition dans le cadre d'une « observation-participante » (Peretz, 2001). L'investigation des pratiques de coordination des acteurs en situation posent de nombreux problèmes méthodologiques que nous proposons de regrouper en trois registres. Les questions liées à l'acceptation " pleine " et " entière " d'un terrain qui permet une telle investigation. II s'agit en premier lieu d'avoir un accord de principe en matière d'accès au terrain. Mais, il s'agit surtout de pouvoir construire dans la durée, un positionnement du chercheur dans l'organisation qui permette de lui trouver une place en tant qu' " observateur-participant " à une situation de coordination (Favret-Saada, 1977 ; Girin, 1990). De plus, cette place doit lui offrir d'avoir les libertés nécessaires pour une investigation approfondie qui peuvent obliger des incursions dans les sphères du " secret " aussi bien que du "privée ». Les questions liées à une investigation d'une pratique située qui suppose d'investir une « connaissance en acte » au sens de Piaget (1974), ayant un caractère non conscientisé au sens de Bourdieu (1990) ou tacite au sens de Polanyi (1996). Les résultats des chercheurs ayant construit des outils d'investigation des pratiques situées (Theureau, 1992 ; Vermersch, 1996 ; Clot, 


\section{Pratiques de coordination d'un collectif informel en situation extrême : une étude de cas « ancrée » au Groenland}

1999 ; Rix \& Lièvre, 2008) conduisent à reconnaitre le caractère nécessaire mais insuffisant, d'une part, d'une observation de la pratique d'un acteur et d'autre part, d'un entretien avec ce même acteur sur cette même pratique. II s'agit alors de proposer un outil qui permette de les associer. Les questions liées à une investigation d'une situation d'interaction qui suppose de pouvoir mettre en relation chaque action individuelle avec l'action collective. Une question sans réponse parce qu'il est quasiment impossible pour un chercheur d'investir à la fois la situation de chaque individu et la situation collective. Or, si on emprunte le registre des actions individuelles comment remonter au niveau du collectif ? Si on emprunte le registre de l'action collective comme une totalité comment revenir sur chaque action individuelle ? Une solution consiste alors à mobiliser deux chercheurs : l'un ayant en charge les actions individuelles et l'autre l'action collective.

Ainsi, nous avons construit un dispositif qui regroupe deux chercheurs ayant deux postures différentes mais complémentaires. Un chercheur a une expérience confirmée d'expéditeur polaire, l'autre est une personne novice en la matière. Tous deux participent à une même expédition -de l'idée du projet, en passant par les divers temps de préparation et la réalisation effective, jusqu'au retour- et sont intégrés à l'équipe. Cependant, chacun adopte des rôles, des attitudes, des manières d'interagir particuliers -si l'un, expérimenté, est acteur à part entière, l'autre, novice, reste plus en position d'observateur- relativement à deux objectifs distincts : d'une part, rendre compte d'une réalité partagée par un collectif, d'autre part, entrer dans le monde de pratique singulier de chaque acteur appartenant à ce collectif. Ainsi deux types de matériaux ont été recueillies : a) des données issues du journal de bord d'un chercheur dont l'objet était de rendre compte du déroulement de l'expédition du point de vue du collectif, b) des données provenant des entretiens conduits par un autre chercheur sur l'expérience vécue de chacun des co-équipiers sur des moments particuliers de l'expédition. Le premier chercheur a développé une posture de participation-observante. Pour réaliser son journal de bord, divers matériaux ont été utilisés : des notes écrites au jour le jour, des enregistrements vidéo en situation assortis de commentaires, des interviews réalisés soit pendant l'expédition, soit après. Le deuxième chercheur a développé une posture d'observation-participante qui permettait d'assister au déroulement de l'expédition mais de conserver une certaine distance par rapport au collectif afin de pouvoir bénéficier des confidences des uns et des autres. II s'agissait surtout de pouvoir réaliser après l'expédition des entretiens centrés sur des moments particuliers en fonction des objectifs de la recherche engagée. La technique utilisée est celle des entretiens en "re situ subjectif » (Rix, Biache, 2004 ; Rix-Lièvre, 2010) qui suppose un enregistrement en vidéo en situation, au préalable. Ce dispositif fait parti des techniques nommées « self confrontation method »(Rix-Lièvre, Lièvre, 2009). Nous avons détaillé ailleurs cette méthodologie (Lièvre, Lièvre-Rix, 2009 ; Rix-Lièvre, Lièvre, 2010). 
Dans le cadre de cet article, nous avons mobilisé a) la description du déroulement de l'expédition sous l'angle du collectif en se centrant particulièrement sur les moments liés à la question de la sécurité ours, b) les investigations relatives au vécu du chef d'expédition en référence à la sécurité ours à des moments particuliers, c) un entretien réalisé pendant l'expédition sur la question des compétences dévolues au chef d'expédition d'un point de vue générique.

Nous ciblons ici dans notre volonté de rendre compte des modalités de coordination mises en œuvre par les acteurs en situation sur le domaine de la gestion de la sécurité ours mais qui suppose auparavant de saisir la coordination sur l'ensemble du périmètre de l'expédition. La question de la sécurité vis-à-vis des ours dans ce type de projet est un domaine sensible puisqu'il touche à la sécurité physique des acteurs en cause. Nous proposons dans une première partie de rendre compte du déploiement de la vie organisationnelle de l'expédition en développant plus précisément les moments relatifs à la gestion de la sécurité " ours », dans un deuxième temps, nous dégageons les registres clés à même de rendre compte des mécanismes de coordination en situation. En conclusion, nous positionnons ces résultats par rapport à la littérature de la coordination et en réponse à la question de recherche affichée dans notre introduction.

\section{Le déroulement de l'expédition Groenland}

Nous proposons de rendre compte du déroulement de l'expédition en privilégiant les aspects lies à la sécurité ours à partir du journal de bord du chercheur ayant investi le collectif. Mais les grandes étapes du projet sont définies à partir de l'entretien réalisé avec le chef d'expédition qui distingue d'une manière générique dans une expédition trois phases : conception, préparation et réalisation auxquelles il associe, pour chacune d'entre elle, des fonctions particulières et donc des compétences critiques distinctes pour le chef de projet. Pour la première phase qui consiste selon lui à inventer un projet original d'expédition, il insiste sur la curiosité, l'imagination, l'ingéniosité que doit posséder un chef d'expédition et du travail laborieux à réaliser bien en amont sur le plan bibliographique à partir d'ouvrages, de cartes, de photos. Pour la seconde phase, c'est le sens de l'organisation qui doit prévaloir : réunir une équipe et se distribuer les rôles selon les grands problèmes : transport, administratif, matériel, progression, financier. Enfin pour la phase de réalisation de l'expédition proprement dite, la compétence du chef d'expédition est principalement l'autorité assortie de compétences techniques pour conduire la progression et lui permettre de ne pas être à la " traîne » dans certains passages et de ne pas apparaitre comme disqualifié visà-vis du collectif.

Cette expédition s'est déroulée sur une période de deux ans et demie en incluant l'idée du projet jusqu'à la phase de clôture des comptes et du retour d'expérience. L'idée a cheminé pendant une année pour arriver à prendre la forme d'un projet 


\section{Pratiques de coordination d'un collectif informel en situation extrême : une étude de cas « ancrée » au Groenland}

qui va donner lieu à une période de préparation d'une année. Le déroulement sur le terrain couvre une période de deux mois. Le bouclage est réalisé rapidement dans le mois qui suit le retour en France de l'expédition.

\subsection{La première phase : la conception}

\subsubsection{De l'idée au projet : une traversée inédite de 800 km à ski au Groenland}

L'idée de cette expédition remonte à une rencontre entre d'une part, deux amis Joël et Gilles et d'autre part, Pierre à l'occasion d'une manifestation sur les expéditions polaires à Paris. Joël et Gilles ont l'habitude de partir ensemble depuis longtemps pour des expéditions sportives à ski, la rencontre avec Pierre leur donne l'envie de partir ensemble tous les trois à la prochaine occasion. Ils semblent partager les mêmes attentes en matière d'expédition que l'on peut décliner sur le thème de l'exploration et la découverte. Une année plus tard, ils se retrouvent chez Joël à l'occasion d'une soirée et décident de réaliser une traversée au Groenland. Pierre est venu avec son amie Cloé qui est aussi partante pour ce genre d'aventure. Depuis longtemps, Joël fait des expéditions au Groenland, mais il n'avait jamais entrepris cette traversée. C'était un projet qu'il avait en tête depuis de nombreuses années. II a déjà toute la documentation et les contacts pour réaliser ce projet. L'itinéraire choisi, de l'ordre de $800 \mathrm{~km}$, n'est pas « classique ». C'est un itinéraire qui n'a jamais été parcouru à ski bien que ne présentant pas de difficultés particulières. C'est une expédition sportive qui se fera en autofinancement. Par contre, le chef d'expédition assure de pouvoir avoir de « bon prix » sur du matériel qui devrait être acheté par les uns et les autres pour l'expédition en question.

\subsubsection{La faisabilité du projet : une documentation à compléter et l'autorisation du Centre Polaire Danois}

Joël a eu une bonne connaissance du Groenland. II a fait déjà de nombreuses expéditions aussi bien sur la côte est que sur la côte ouest. La seule chose qui lui manque ce sont des photographies aériennes pour repérer d'une manière précise tels passages ou tels autres qui ne peuvent être appréciés avec les cartes disponibles. L'autre problème réside dans l'obtention d'un permis d'expédition par le Centre Polaire Danois. Mais Joël a déjà effectué ce type de demande de permis d'expédition de nombreuses fois au même organisme. II est rompu à l'exercice. Pour le transport, il prend contact avec son agence habituelle qui est spécialisée sur ce type de voyage et avec laquelle il travaille depuis de nombreuses années. Cette même agence va aussi s'occuper de tout ce qui est l'hébergement sur place. Enfin il a l'accord d'un magasin spécialisé dans les articles de montagne pour obtenir une réduction de $30 \%$ sur tout le matériel acheté pour son expédition. Reste à régler le délicat problème du recrutement de ses co-équipiers. 


\subsection{La deuxième phase : la préparation}

\subsubsection{Le recrutement des co-équipiers : une tâche délicate et sensible quant à l'issue du projet}

Le recrutement des co-équipiers est une étape essentielle et délicate à réaliser comme le rappelle à de nombreuses reprises le chef d'expédition. En effet, le choix des co-équipiers va fortement conditionner le " bon » déroulement de l'expédition lors de sa mise en œuvre sur le terrain. Dans un contexte hostile, la bonne coopération entre les équipiers est vitale. II déclare avoir eu par le passé de nombreuses difficultés sur ce sujet. II est opposé à la constitution de collectif qui se retrouve véritablement pour la première fois dans l'avion juste avant de partir en expédition. L'idéal pour ce genre d'expédition serait de partir à six coéquipiers pour des raisons de sécurité. En effet la chute dans une crevasse d'un équipier qui est un risque que l'on ne peut éliminer sur ce genre d'itinéraire est quelque chose qui peut être abordée plus sereinement à quatre ou à cinq qu'à trois pour organiser un secours. S'il faut organiser des tours de garde la nuit pour éviter l'intrusion d'un ours blanc dans le camp, ceci est une chose plus facile à envisager à six qu'à trois. Nous sommes quatre, il faut trouver deux autres compères. Joël pense que deux autres amis pourraient être intéressés par le projet, mais ils ne sont pas libres sur la période. Pierre connaît aussi une personne avec laquelle il est déjà parti en expédition qui pourrait être intéressée par le projet. Joël est contrarié. II n'est pas très favorable à la venue de l'ami de Pierre : il ne le connaît pas. II a eu par le passé de nombreux problèmes en invitant des gens qu'il ne connaissait pas à participer à l'expédition. Joël a diffusé l'information de l'expédition à divers contacts, mais au final Joël proposera de partir à quatre. Il écarte deux candidats avec lequel il a eu des problèmes lors d'une expédition, il y a quelques années : une opposition quant à l'itinéraire proposé qui avait été difficile à gérer.

\subsubsection{Planification de la préparation : le matériel, la sécurité ours, le collectif, la nutrition}

Joël va distribuer les différentes missions en fonction de sa manière habituelle de faire en distinguant : transport, administratif, matériel, progression, financier. La bonne réalisation de chacune de ses missions fera l'objet d'un suivi précis de sa part. Le premier problème à régler est celui du choix du matériel. Les débats sont animés. Il y a des choix individuels et des choix collectifs. Le dialogue va s'effectuer pendant un mois. Un accord est enfin trouvé. Joël et Pierre vont diverger sur le choix des chaussures et des fixations sur les skis. C'est un équipement type haute montagne qui est retenu (crampons, piolet, cordes, poulies-bloqueurs...) et des skis légers de randonnée nordique avec des carres métalliques. Le campement s'effectue par tente de deux. Joël propose que l'équipe se retrouve pour acheter ensemble le matériel. L'équipe prend une journée pour réaliser l'opération. Le second problème qui va être abordé, lors d'une réunion, est celui de la protection 


\section{Pratiques de coordination d'un collectif informel en situation extrême : une étude de cas « ancrée » au Groenland}

contre les ours blancs. Les avis sont très partagés. Chacun relate les diverses expériences qu'il a eu avec l'ours blanc et les différentes techniques utilisées. De toute façon, on emmène un fusil. C'est l'outil obligatoire à avoir avec soi dans ce genre d'expédition. La question se pose alors de l'éventualité d'avoir deux fusils : un par tente. "Non, c'est trop dangereux, il faut un seul fusil et c'est moi qui le prends " déclare Joël. Et pour la nuit en matière de sécurité ours comment on fait ? De nombreuses possibilités existent : tour de garde, système d'alerte autour du camp... Joël tranche le problème en proposant de prendre des chiens : une technique qu'il a déjà utilisée. Les autres n'ont jamais utilisé des chiens et sont sceptiques. Les chiens peuvent aussi attirer les ours. Joël précise que c'est lui et Gilles qui s'occuperont des chiens pendant l'expédition. L'affaire est conclue. II décide d'acheter deux chiens sur place. Le troisième élément de préparation tient au fait de partir ensemble pour une sortie de ski de randonnée pour voir comment le collectif peut fonctionner. Ils se retrouvent pour un week-end en montagne. De bonnes conditions de neige, un temps superbe, une belle randonnée à ski, l'atmosphère est sereine : le groupe fonctionne. Un diner légèrement gastronomique pour clôturer la sortie. Pour la préparation de la nourriture chacun procède comme il fait d'habitude en tenant compte des contraintes de poids dans l'avion. De toute façon en situation chacun tire sa pulka avec son propre matériel et sa propre nourriture. Joël a établit un itinéraire relativement précis avec des prévisions de camp pour chaque soir. II sait bien que cet itinéraire et surtout l'établissement des camps peuvent être remis en cause pour de nombreuses raisons, mais de cette façon, en situation il sait où il en est : s'il a pris du retard, s'il est en avance, s'il faut modifier le tracé au vu de l'avancée ou d'autres problèmes qui peuvent émerger. Le rendez-vous est donné de se retrouver à l'aéroport de Roissy Charles de Gaulle le jour J avec les bagages.

\subsection{La troisième phase : la réalisation de l'expédition sur le terrain}

\subsubsection{Des bagages en rade : une source de controverses entre Joël et Pierre}

Le voyage s'effectue sans problème sauf qu'une partie des bagages n'a pas suivi les passagers. Ces derniers arrivent sur place le jeudi. Ils espèrent pouvoir récupérer les bagages manquants dans l'avion le samedi matin. Ils finissent les préparatifs du matériel. Les derniers achats de provision sont faits ; sachant qu'il n'y aura plus aucune possibilité de se ravitailler après. Ils vont à l'arrivée de l'avion du samedi : pas de bagages. Joël veut partir. Pierre n'est pas d'accord. Pierre ne veut pas partir sans les bagages manquants dont l'un d'entre eux contient l'ensemble de la nourriture de son amie. Elle ne pourra pas tout retrouver sur place en matière alimentaire et en plus il faut tout reconditionner par ration journalière pour une expédition de 40 jours. Pour occuper la journée libre de 
dimanche, il est proposé de faire une ballade à ski. Le lundi matin les bagages sont là. Joël décide de partir dans le même mouvement car il fait beau.

\subsubsection{La présence des ours, un démarrage difficile avec un froid intense, Joël se gèle un doigt}

Lors du déplacement en motoneige pour rejoindre le point de départ du périple, l'équipe aperçoit des ours blancs. Ils sont avertis, le danger est réel. Ils font peu de kilomètre à ski. C'est le premier jour. Ils posent le camp et se couchent rapidement. Le deuxième jour, le temps est beau mais il y a un vent violent. La progression est difficile avec les chiens qui n'ont jamais fait ce type d'exercice : marcher à coté d'un skieur, trainer un petit traineau qui contient leur nourriture. II y a 300 mètres de dénivelé pour atteindre le col ce qui est toujours une épreuve physique lorsque l'on traine une pulka en moyenne de $90 \mathrm{~kg}$. Joël est parti tôt ce matin pour repérer un passage et il faisait froid. II a un pouce qui a gelé. II ne dit trop rien. Mais en début d'après-midi, le vent souffle encore plus fort et Joël n'a pas réussi à réchauffer son doigt. L'équipe s'inquiète. Pierre décide de planter le camp immédiatement pour que Joël puisse se réchauffer.

\subsubsection{Incompréhension entre les équipiers qui conduit à la perte des chiens, le bricolage pour pallier à leurs absences}

Le lendemain Joël repart comme si de rien n'était. II part en avant avec un chien sans mot dire. Les autres le suivent, puis le rattrapent. Ils arrivent à un passage délicat sur une rivière de glace qui nécessite de mettre les crampons. Les chiens ont peur : ils glissent. Joël et Gilles qui devaient s'occuper des chiens sont devant. De fait, Pierre et son amie récupèrent les chiens. La progression est lente. Ils arrivent sur une rupture de pente dans la glace : un fossé de 2 à 3 mètres. Pierre s'engage lentement avec un chien et glisse. Pour ne pas blesser le chien il le lâche et l'envoie en direction de Joël qui attendait en bas. Au lieu de se précipiter sur lui Joël déclare : il ne faut pas s'en occuper! II ne va pas aller bien loin. Voyant le premier chien s'enfuir, le second se débat et réussit à s'enfuir. Les chiens sont partis. Ils ne reviendront pas. Tension entre les co-équipiers après cette histoire de chien. L'équipe s'inquiète sur le fait de ne plus avoir de sécurité ours. Joël minimise le problème et ne semble pas s'en préoccuper. Pierre a emporté avec lui du matériel de sécurité héritée d'autres expéditions : des fusées d'alarme, du fil, des élastiques de caoutchouc. II fabrique une clôture anti-ours qui va permettre de protéger le camp de la venue intempestive d'un ours blanc la nuit.

\subsubsection{La chute à l'eau du chef d'expédition}

Le lendemain Joël démarre la journée sans aucune consigne de coordination. Ils abordent une rivière en glace dont la pente est très faible. Joël prend l'option crampon. Comme il le répète à de nombreuses occasions, un montagnard dés qu'il voit de la glace : il met les crampons. Pierre propose une autre solution qu'il a déjà expérimentée avec les bottes Sorel : l'adhérence est suffisante pour progresser dans un mouvement proche du pas alternatif en ski de fond en profitant 


\section{Pratiques de coordination d'un collectif informel en situation extrême : une étude de cas « ancrée » au Groenland}

de l'appui des bâtons munis de pointe carbone. Les deux autres empruntent la technique de Pierre. Ils progressent très rapidement et Joël ne peut les suivre. Ils s'arrêtent pour boire, manger et attendre Joël. De plus la partie qui arrive n'est pas engageante : la glace change de couleur et la frappe du bâton sur la glace résonne bizarrement. Joël arrive, il les dépasse et décide de ne pas s'arrêter. Le voila devant eux. La glace s'effondre sous ses pas. II est à l'eau. II ne sait pas nager. Toute tentative pour reprendre pied sur la glace est vouée à l'échec avec les crampons qui cassent la glace. Les autres se tiennent à l'écart de cette glace fragile. Ils rejoignent le bord de la rivière pour lui envoyer une corde. Ils le hissent et le font glisser jusqu'à la rive : il est trempé. II se déshabille entièrement. II faut qu'il se sèche complètement. II n'a pas de vêtements de rechange : il a emmené le strict minimum pour l'expédition. Les autres lui trouvent du rechange dans leurs affaires. II se change entièrement. Pierre et son amie avait emporté deux paires de chaussures : l'une pour le ski, l'autre pour la marche. Ce qui avait fait l'objet de controverses entre eux, Joël ne voyant pas l'utilité de ce doublon. Et heureusement Joël rentre dans l'une des chaussures. Ils progressent une heure pour réchauffer Joël et montent le camp. Pierre utilise un réchaud qui permet de chauffer la tente car il peut fonctionner à un faible débit. II chauffe la tente et installe le séchage du matériel. Les chaussures de Joël vont mettre une vingtaine d'heures à sécher avec moultes manipulations. Aussi le lendemain, il est décidé de rester la journée au camp pour finir de faire sécher les affaires de Joël.

\subsubsection{Des choix sans concertation et une routine qui s'installe progressivement}

Le surlendemain, ils repartent sans consigne pour la journée de la part de Joël qui part devant. Ils arrivent à coté d'un sommet qu'ils avaient prévu de gravir et de descendre en ski. Les conditions sont excellentes en neige et il fait beau. Ce sommet est classique dans le milieu polaire. Joël a décidé que le sommet n'est plus à l'ordre du jour au vu du temps que nous avons perdu ; il prend cette décision sans s'en expliquer et sans demander l'avis des autres équipiers. Ils repartent le lendemain toujours sans consigne. L'expédition progresse maintenant d'une manière régulière. Quelques problèmes d'itinéraires à deux endroits qui vont être source de tension entre les membres de l'équipe. Joël continue à progresser devant le matin sans donner de consigne pour la journée. Les autres se sont fait une raison et suivent. Lors d'un ravitaillement prévu avec des Inuits, Joël achète un chien qui sera pris en charge complètement par Gilles. Le rythme de progression n'est pas suffisant. Ils n'arriveront pas à boucler la traversée avec la nourriture prévue et il y a la contrainte de l'avion pour le retour en France. Joël décide de bloquer les lieux de camp et de s'obliger à atteindre le lieu de camp à chaque étape quelque soit le nombre d'heure de progression. Ceci est possible puisqu'ils sont en période de jour permanent. Les deux dernières étapes sont effectuées avec de longues journées de progression de 15 à 16 heures. Ils prennent l'avion pour le retour en France à la date prévue et fixent ensemble la date de la réunion de bouclage qui doit se dérouler un mois après l'expédition. 


\subsubsection{Le bouclage de l'expédition}

Projection de diapositives, échange de photos, une belle soirée autour d'un bon repas. Ils échangent sur leurs vécus. Pierre et Gilles sont prêts à repartir avec Pierre et son amie. Pierre et son amie sont plus hésitants. Ils recherchent une vie collective plus intense dans une expédition. Même si il y a un chef d'expédition : les décisions doivent faire l'objet de véritables échanges entre les coéquipiers. Ils regrettent de ne pas avoir été associés à la décision de ne pas faire le sommet alors que les retards accumulés étaient de plus largement du à Joël. Ils ont trouvé aussi Joël négligeant sur sa gestion de la sécurité ours.

\section{Mode de coordination « ancrée » relative à la sécurité ours}

Nous proposons de rendre compte des modes de coordination ancrée au sens de Glaser et Strauss (1967) de cette expédition Groenland au plus prés de ce qui a été vécu par le collectif, mais aussi par le chef d'expédition et en intégrant ses propres principes d'organisation issus de sa grande expérience des expéditions polaires. II est possible dans un premier temps dégager les points suivants sur la question de la coordination en générale sur le périmètre de l'ensemble de l'expédition et dans un deuxième temps sur la question de la sécurité ours proprement dite.

\subsection{Modes de coordination sur le périmètre global de l'expédition}

Il est possible de dégager les points suivants sur la question de la coordination en générale sur le périmètre de l'ensemble de l'expédition : la surdétermination de la coordination par le chef d'expédition, des modes distincts de coordination en fonction des phases du projet, une phase de réalisation délicate pour le maintien de l'autorité du chef d'expédition, un collectif informel en phase de création de routines organisationnelles.

\subsubsection{Des modes de coordination surdéterminées par le chef d'expédition}

Le rôle joué par Joël le chef d'expédition est déterminant dans le mode de structuration de la coordination tout au long du déroulement du projet. Ceci tient à plusieurs raisons. Premièrement, Joël est l'initiative du projet, et plus précisément, cette traversée est son projet. II est le garant de la réalisation du projet, mais aussi de son esprit tout au long de son déroulement. Deuxièmement, Joël a une grande expérience des expéditions polaires. Cette expertise s'est acquise au fur et à mesure de la trentaine d'expédition qu'il a conduite. Il développe une manière très personnelle d'organiser une expédition. II a publié des articles et même des ouvrages de référence sur la question, il a formé dans des associations de montagne de nombreux «novices » à ces questions. On pourrait dire qu'il a 


\section{Pratiques de coordination d'un collectif informel en situation extrême : une étude de cas « ancrée » au Groenland}

construit un schème " organisation d'une expédition polaire » au sens de Piaget c'est-à-dire d'un invariant opératoire dans la manière de conduire une expédition (Vergnaud, Recopé, 2000). Troisièmement, le collectif qui se réunit pour cette expédition n'a jamais fonctionné ensemble. On est en présence de deux binômes ayant construit séparément des routines organisationnelles en matière d'expédition polaire qui peuvent être source de tensions entre les équipiers. Joël est vigilant de conserver son leadership dans cette histoire pour conduire à bien cette expédition. II va réaliser par lui-même la phase de conception, il va organiser d'une manière précise la phase de préparation en répartissant les taches et en contrôlant leur réalisation, et enfin il va planifier le déroulement chronologique de cette phase. Pour la phase de réalisation, il va tout faire pour conserver le leadership de l'expédition.

\subsubsection{Des modes de coordination en rapport avec des problèmes de gestion spécifique en fonction des phases de l'expédition}

A chaque phase du déroulement du projet, le chef d'expédition identifie des problèmes différents de gestion qui vont faire l'objet de mode de coordination distinct. Dans la première phase où le problème principal est de l'ordre de la conception, le chef d'expédition va réaliser par lui-même cette étape en mobilisant des acteurs extérieurs à l'équipe projet. La phase de préparation va être conduite sur le modèle canonique de la coordination-organisation telle qu'il est exprimé d'une manière synthétique par Alsène et Pichault (2004) à partir des travaux pionniers de Fayol. Selon eux, il est possible de dégager trois registres en matière de coordination : le registre de la répartition des ressources et des taches dans une logique à la fois d'efficience et de répartition égalitaire, le registre de la mise en cohérence dans le temps des actes et enfin le registre de l'orchestration des activités sous l'angle d'un collectif propre à permettre, par exemple, l'émergence de synergies. Joël adopte un modèle de coordination hiérarchique pour la répartition des tâches et les prises de décision, sauf pour la question du matériel où une approche plus participative est mise en œuvre. Le collectif fera l'objet d'un temps spécifique, sous la forme d'une sortie en ski, décidé et organisé par Joël. Enfin pour la troisième phase celle de la réalisation, où l'adaptation doit prévaloir à toute planification, met le chef d'expédition dans une posture délicate qui va le conduire à porter une grande attention à la question du maintien de son autorité. Nous allons revenir plus dans le détail des problèmes qui se posent à un chef d'expédition dans ce type contexte.

\subsubsection{Une phase de réalisation délicate pour le chef d'expédition}

La phase de réalisation de l'expédition amène le chef d'expédition dans une situation de gestion spécifique. Joël de par son expérience est très conscient de cette situation. Sur le terrain, le chef d'expédition devient un équipier comme un autre, et il va devoir être un équipier exemplaire. Et s'il n'est pas exemplaire, son autorité peut être remise en cause. Par ailleurs, c'est lui qui est responsable de l'itinéraire et de la progression en tête qui peut constituer toujours une source 
d'erreur et ainsi, il risque ainsi d'être mis en défaut dans ce rôle. Bien que de nombreuses choses aient été planifiées, préparées et anticipées à l'avance du fait de son expérience, il sait bien qu'en situation tout peut être remis en cause à n'importe quel moment et que des évènements imprévisibles vont surgir qui peuvent amener des controverses au sein des équipiers où son autorité peut être encore mise à mal. Listons ces événements dans le cours de l'expédition qui peuvent être autant de remise en cause de son autorité : son doigt gelé qui oblige de monter le camp rapidement; son passage à l'eau alors qu'il en tête sur cette rivière en glace, crampons au pied qui va imposer un temps de séchage et donc d'immobilisation non négligeable, les chiens qu'ils ne rattrapent pas, sa non-préoccupation d'une solution de rechange à la perte des chiens... Cette préoccupation n'est pas propre à Joël en tant que chef d'expédition. Des chefs d'expédition contemporains et emblématiques comme Eric Tabarly, Jean Louis Etienne, Nicolas Vanier ont tous fait la désagréable expérience de l'expédition qu'il ne contrôlait plus sur le terrain, lors de leurs premières expériences en tant chef d'expédition et ils ont cherché à développer des moyens pour pallier à ce problème crucial.

Pour faire face à cette préoccupante question, Joël va faire attention a deux choses dans les phases amont du projet : le choix des équipiers et l'attribution du fusil.

\section{Le choix des équipiers}

Joël va porter une grande attention pour assoir son autorité dans la phase de progression au recrutement des équipiers en amont. Le recrutement des équipiers semble se réaliser selon trois critères : la capacité de l'acteur à suivre physiquement et techniquement l'expédition, à s'intégrer à l'objectif de l'expédition qui est de l'ordre de l'exploration, et à accepter l'autorité du chef d'expédition. Et ce dernier point est très important puisque tous les candidats proposés par Pierre seront écartés, car ils présentent le risque de voir son autorité contesté par un collectif. Pierre a aussi une expérience en tant que chef d'expédition (15 expéditions), inférieure à celle de Joël, mais suffisante pour discuter les choix de Joël et proposer des alternatives. Ce fait va se trouver corroborer par la suite sur différents aspects.

\section{L'attribution du fusil}

Joël va porter une grande attention pour assoir son autorité dans la phase de progression au fait qu'il soit le détenteur du fusil pour se protéger des ours. Lors de la réunion qui a présidé aux divers choix techniques, la question a été tranchée d'une manière unilatérale : c'est Joël qui a déclaré qu'il serait détenteur du fusil, sans justifier cette prise de position. A aucun moment le choix de qui aurait le fusil en situation n'a été abordé en termes de capacité à tirer. Et lorsque Pierre a proposé d'avoir deux fusils, Joël a écarté de suite cette éventualité car trop dangereuse, selon lui. Pierre a réalisé de nombreuses expéditions avec 


\section{Pratiques de coordination d'un collectif informel en situation extrême : une étude de cas « ancrée » au Groenland}

deux fusils sans que cela ne pose le moindre problème. Par contre, en devenant l'unique détenteur du fusil, il détient un outil d'une grande importance quant à la construction et au maintien de son autorité dans la phase de progression. Avec l'unique fusil sur l'épaule de Joël, tout équipier se trouve en quelque sorte obligé d'être assez proche de lui. S'il part devant, les autres vont devoir le rejoindre, s'il est distancé par le groupe, les équipiers vont devoir l'attendre.

\subsubsection{Un collectif qui n'a jamais fonctionné ensemble en situation}

II s'agit d'un collectif informel qui se réunit pour la première fois à l'occasion de cette expédition. Le chef d'expédition a construit un itinéraire et a identifié des lieux de camp possibles. Il a établi une moyenne journalière de progression à ski pour savoir où il en est à tout moment. II est en retard. II est en avance. De toute façon, il faut s'adapter. Le plan est incomplet par définition, et il doit être une ressource pour l'action. Nous sommes ici dans une perspective proche des travaux de Suchman (1987). Un certain nombre de rôles ont été distribués. C'est Joël en tant que chef d'expédition qui fait l'itinéraire et qui assure a priori la progression en tête. C'est Joël et Gilles qui prennent en charge la sécurité ours en se chargeant des chiens aussi bien dans la phase de progression que pendant le campement. Pierre et Cloé sont autonomes en ce qui concerne leurs tentes et leurs nutritions. Suite à certains incidents, c'est Pierre qui a pris le leadership du groupe comme par exemple en décidant de monter le camp suite au doigt gelé de Joël. Certains jours, Joël et Gilles n'ont pas assuré la prise en charge des chiens en situation de progression, c'est Cloe et Pierre qui s'en sont chargés, ce qui a donné lieu à des problèmes. Après le départ des chiens, Joël ne s'est pas préoccupé d'essayer de palier à cette carence, c'est Pierre qui a bricolé un système et qui va le mettre en œuvre systématiquement. L'organisation quotidienne du déroulement de l'expédition n'est pas calée à l'avance. C'est Joël sur le terrain qui décidera. II impose en fait son standard d'organisation à l'expédition.

\subsection{La coordination proprement dite liées à la sécurité " ours »}

Le récit de l'expédition indique que la question de la sécurité ours est abordée dans la phase de préparation lors d'une réunion organisée par le chef de l'expédition. Le choix qui a été retenu finalement par le chef d'expédition, repose sur deux outils : la présence de chiens tout au long du déroulement de l'expédition qui doivent écarter les ours blancs ou en dernier ressort prévenir de leurs arrivées éventuelles et d'un fusil qui constitue une arme destinée à être utilisée en cas d'attaque caractérisée de l'animal. Si la question des chiens a été effectivement débattue par les différents équipiers, rien ne dit que la décision n'avait pas été déjà prise par Joël et Gilles avant la réunion. Sachant que le fait que Joël et Gilles prenaient en charge complètement la question des chiens : en progression, lors des campements, la question de la nourriture... devait balayer les différents états d'âme des autres équipiers. Quant à la question du fusil, la discussion a été vite close par Joël : on prend un « seul » fusil et c'est moi qui le porte. Joël avec l'aide 
de Gilles prend en charge complètement le problème de la protection contre les ours. C'est lui qui détient l'arme et il gère les chiens avec ce co-équipier. Pendant la phase de réalisation sur le terrain, Joël ne semble pas porter une grande attention à la gestion des chiens et commet des erreurs d'appréciation qui vont amener à la perte définitive des chiens. Devant ces différentes négligences, les autres coéquipiers réagissent vivement et tentent de bricoler un système d'alarme avec des fusées qui seront installées chaque soir autour du camp.

Les entretiens menés avec une technique d'auto-confrontation, avec Joël relatif à son vécu en situation en rapport avec la question de la sécurité ours permettent d'apprécier autrement son rapport à cette question. Pour lui, le risque lié aux ours dans cette région est minime. L'option « chien » a été retenue pour satisfaire les préoccupations des autres équipiers et surtout celle de Gilles. Pour lui, le seul moment où il y a des risques d'ours est lorsque sa présence est avérée, et dans cette situation, le seul outil qui prévaut est le fusil et l'équipe doit progresser d'une manière ramassée derrière le fusil. Ainsi, nous avons pu documenter un moment de ce type en situation qui est une objectivation du vécu de Joël dont nous pouvons rendre compte de la manière suivante : "Dans ce fond de fjord, Joël sait qu'il y a des ours : il a vu des tanières à cet endroit-là. Les traces de pas dans la neige et les restes d'un phoque confirment cette éventualité. Tout en progressant, il guette. Un ours peut être caché derrière un bloc de glace : on risque de le voir au dernier moment, quand il charge. II faut être prêt à intervenir. Le fusil est sur la pulka de Joël. Pour la sécurité de tous, il vaut mieux être groupé et derrière le fusil. Joël l'a indiqué à tout le monde, mais les autres continuent à rester devant, ce qui l'agace. Tout en progressant à son rythme, il reste attentif ». Pour Joël, le risque de l'ours lors de cette expédition n'existe que lorsque celui-ci se manifeste par des traces visibles de sa présence : c'est uniquement dans ce contexte précis qu'il met en œuvre un dispositif de sécurité en rapport avec le fusil qu'il détient. Cette manière de gérer les risques pour Joël est à l'écart du discours qu'il tient en que chef d'expédition devant le collectif et des préconisations afférentes comme par exemple le système de protection permanent assuré par des chiens. Pour cet ancien alpiniste, d'une part, le risque est avant tout vertical. Or l'expédition se déroule à l'horizontal. D'autre part, il a parcouru, dans des expéditions antérieures, des terrains où la population des ours était très importante, et non comme ici résiduel. Mais surtout sa tendance profonde s'exprime dans la contemplation esthétique et solitaire de cet univers et tout ce qui est obstacle à cette aspiration est du temps perdu. En clair, pour lui, le risque d'ours est uniquement avéré lorsqu'il voit des signes tangibles de la présence de l'ours en situation, et seulement dans ce cas la, il se mobilise avec le seul outil qui possèdet une valeur, le fusil.

Le fusil devient un outil exemplaire parce que pour Joël c'est le seul outil valable pour se protéger des ours et c'est lui qui le porte en situation, quelles que soient ses qualités de tireurs en rapport avec les autres équipiers. Le fusil permet 


\section{Pratiques de coordination d'un collectif informel en situation extrême : une étude de cas « ancrée » au Groenland}

d'ordonner la progression en situation de danger avéré, mais aussi il permet d'ordonner la progression en toute situation parce qu'il n'est pas raisonnable pour les autres équipiers d'être trop à l'écart du fusil pendant le déroulement quotidien de l'expédition. Ainsi, le fait pour Joël d'être le porteur du fusil conforte sa position de leader dans la progression. La question de l'attribution du fusil en rapport avec la question de la coordination de l'expédition pendant la phase de réalisation prend une ampleur remarquable quant à la possibilité ou non de conforter la légitimité du chef d'expédition.

\section{Conclusion}

On aurait pu imaginer que si l'on suit les canons des théories de la contingence que ce collectif informel allait se complaire dans l'informel pour faire face à la situation, il n'en est rien. Mieux, tout se passe comme s'il y avait une nécessité de construire une routine organisationnelle partagée qui de fait n'existe pas. Nous rappelons qu'il s'agit d'un collectif informel d'acteurs « amateurs »; n'appartenant à aucune organisation ou institution commune ; n'ayant jamais fonctionné ensemble dans une situation similaire et donc n'ayant pas construit de routine organisationnelle pré-existante ; ne pouvant pas se reposer sur un standard formel codifié de coordination appris collectivement dans une école ou faisant l'objet d'une norme dans une organisation. Pour construire cette routine organisationnelle, le chef d'expédition impose son « standard » de l'organisation d'une expédition polaire : son « invariant opératoire » sur la manière de conduire une expédition, un schème au sens de Piaget, qu'il a construit tout au long de sa longue expérience de chef d'expédition polaire. Plus précisément, comme l'exprime Michel Recopé (2001), il s'agit d'une combinaison de schèmes orientés par l'intentionnalité de l'acteur en référence à l'activité. Ainsi, il poursuit : "Certains expéditeurs polaires seraient caractérisés par la construction de schèmes qui leur confèrent des tendances à la réalisation de soi par la réalisation d'expédition indissociables d'un idéal et de valeurs fortes, de besoins et de mouvements ». Le schème est un modèle cognitif opératoire issu des travaux de Piaget (1936), repris par Vergnaud (1996), qui permet de rendre compte des modalités d'organisations invariantes de l'activité humaine pour une classe de situation donnée (Vergnaud, Recopé, 2000). Des liens ont été faits entre cette notion de schème et la notion d'habitus de Bourdieu en tant que disposition à agir non conscientisée, incarnée et orientée (Lahire, 2001). Joël a un palmarès éloquent : la conduite d'une trentaine d'expédition, la rédaction de nombreux compte-rendu d'expédition pour des revues spécialisées, la rédaction d'un ouvrage de référence sur l'organisation des raids à ski dans le milieu polaire, la prise en charge de module de formation dans des associations sur ces questions... Nous pourrions traduire l'intentionnalité de Joël en termes d'exploration esthétique et solitaire du monde polaire.

Si l'imposition de ce standard ne pose pas de problème particulier dans la phase de conception par définition, puisqu'elle est réalisée exclusivement par 
Joël et à l'écart de l'équipe-projet. Si l'imposition de ce standard fonctionne correctement dans la phase de préparation parce qu'il donne un cadre très précis à ce qui va faire l'objet d'une réelle discussion entre les acteurs et ce qui va relever directement de la décision du chef d'expédition. Comme il l'exprime très clairement dans l'entretien que nous avons eu avec lui sur l'organisation générique d'une expédition : il s'agit dans cette phase de préparation de réunir une équipe et de se distribuer les rôles selon les grands problèmes : transport, administratif, matériel, progression, financier.

II va jouer sur différents registres en matière de leadership pour organiser la coordination des acteurs. II va être hiérarchique sur la manière de décomposer les problèmes et de construire le planning de la préparation. II va être assez directif sur qui va faire quoi dans cette organisation. II va contrôler d'une manière très précise si les missions des uns et des autres sont bien réalisés II va être plutôt participatif sur la question du matériel. II y aura des discussions entre Joël et Pierre, mais qui sont finalement des controverses de standard entre celui de Joël et de Pierre sur la manière de conduire une expédition, et dans le registre du matériel. Par contre, deux registres pour Joël dans cette phase de préparation vont faire l'objet d'une attention essentielle car ils conditionnent la possibilité de conserver sa légitimité en tant que chef d'expédition dans la phase de réalisation où tout peut arriver : le recrutement et l'attribution du fusil. Ainsi, la question du recrutement relève exclusivement de sa décision personnelle parce qu'il s'agit d'un sujet d'un enjeu considérable tout au long du déroulement de l'expédition sur le terrain, comme nous l'avons évoqué. II faut que les acteurs acceptent son autorité tout au long de l'expédition. L'attribution du fusil pour des raisons de risque qui lui sont propres comme nous l'avons souligné et pour des raisons encore de maintien de son autorité dans la phase de progression ne peut aller qu'au chef d'expédition. En effet, dans cet univers où plane quotidiennement l'irruption possible d'un ours blanc, les équipiers ne seront pas enclins de fait à trop s'éloigner du chef à tout moment de l'expédition ce qui règle quelques problèmes de coordination dans la progression quotidienne : le départ du camp, les pauses pour les repas, l'itinéraire précis à suivre, le choix du campement.

Joël sait très bien que les choses vont se compliquer pour fonctionner de cette manière dans la phase de réalisation parce que le plan sera toujours incomplet, la répartition des rôles sera inachevée et ne sera pas toujours respectée parce que c'est la situation qui prévaut en la matière et qu'il va falloir faire face, qu'il va falloir s'adapter. Dans ce contexte son autorité peut être mis à mal d'autant qu'il est un équipier comme les autres, aussi il se doit être exemplaire pour conserver son autorité et d'autant qu'il détient des rôles relatifs à l'orientation ou la progression en tête où l'erreur est facile. Pierre va prendre le rôle du leader du groupe à différents moments. Joël a son pouce gelé. Pierre organise une rapide montée du camp pour qu'il puisse se réchauffer. Pierre alors qu'il ne devrait pas avoir les chiens en progression, laisse partir un chien, non rattrapé par Joe. C'est Pierre 


\section{Pratiques de coordination d'un collectif informel en situation extrême : une étude de cas « ancrée » au Groenland}

qui bricole une nouvelle sécurité ours alors que c'est Joël et Gilles qui devaient se charger de ce registre. Enfin sur la progression sur la rivière gelée, il y a controverse entre Pierre et Joël sur deux manières de faire. Joël se retrouve isolé dans sa pratique et de plus l'amène à tomber à l'eau. C'est grâce au standard de Pierre qu'il y a d'autres paires de chaussures et d'autres vêtements qui vont permettre à Joël de se réchauffer. C'est Pierre qui organise à sa manière avec l'aide de Cloé le séchage du matériel de Joël dans leur tente. Ainsi on pourrait dire que lorsque Joël se trouve dans une situation délicate, c'est le standard de Pierre qui va s'imposer au groupe.

On pourrait dire que finalement le collectif a fait preuve de résilience organisationnelle au sens de Weick (1993) dans le sens où il a trouvé en situation des solutions pour faire face à des problèmes " aigus » : le pouce gelé de Joël, la perte des chiens pour la sécurité ours, le passage à l'eau de Joël. La résilience organisationnelle chez Weick est un processus permettant l'émergence de solutions individuelles et collectives propre à faire face à une situation de crise et à permettre d'éviter la catastrophe. Ainsi il distingue quatre registres, comme source de la résilience organisationnelle : improvisation et bricolage, système de rôle virtuel, la sagesse comme attitude, l'interaction respectueuse. Nous pourrions approfondir cet aspect, mais ce n'est pas l'objet de ce papier. Mais on pressent qu'effectivement ces différents registres étaient présents dans le cas que nous avons étudié. Mais le faible investissement de Joël sur la question du collectif, le caractère hiérarchique de son leadership à des moments qui auraient du relever d'un choix du collectif, comme par exemple l'abandon de la réalisation du sommet, montre qu'il n'était pas dans cet esprit là et que cette résilience organisationnelle constatée était plutôt une émergence propre du collectif, plutôt que délibérée.

L'investigation des pratiques de coordination en situation extrême, que nous avons réalisée lors de l'expédition Groenland montre le caractère fondamentalement émergent et processuel de la coordination. II y a une situation initiale pour des acteurs donnés, dans un contexte organisationnel spécifié qui permet de comprendre les pratiques de coordination. II apparait différentes types de pratiques de coordination qui vont se succéder dans le temps et qui vont s'articuler entre elles. Ce caractère résolument émergent des pratiques de coordination est aussi le résultat auquel ont abouti Faraj et Xiao ( 2006) dans le travail qu'ils ont faits sur les " pratiques » de coordination dans un centre de traumatologie, en se mettant à distance d'une visée structurelle et contingente de la coordination. L'étude approfondie réalisée dans cet établissement, de premier plan, pendant 18 mois, a dégagé deux types de pratiques alternatives de coordination : une pratique fondée sur l'expertise et une pratique fondée sur la dialogie. Si nous relisons de ce point de vue, notre étude de cas, nous pouvons décrire les pratiques de coordination selon ces deux registres, mais en spécifiant que dans l'expédition Groenland, c'est la coordination par expertise qui 
a fonctionné plutôt que la coordination par dialogie. Par contre, c'est le leader qui surdétermine le choix de la pratique de coordination en situation. Sauf quand ce dernier est mis à mal en situation, en tant que co-équipier, comme c'est le cas lors son passage à l'eau, où c'est une pratique de coordination dialogique qui va se mettre en œuvre spontanément pour le sortir de l'eau, et que c'est l'expertise d'un autre co-équipier, le plus expérimenté qui va organiser dans un deuxième temps la suite des opérations. La nature informelle et émergente du collectif et le fait que le leader soit un équipier comme un autre, conduisent ce dernier de se préoccuper de sa légitimité. Si celle-ci est entamée, il ne pourra pas même s'il le voulait mettre en place une pratique de coordination dialogique. Ce qui apparait comme une différence de fond entre les deux études, c'est la place du leadership dans la coordination qui n'apparait pas comme une préoccupation essentielle dans le cadre du centre de traumatologie et à l'inverse dans le cas de l'expédition polaire, celle-ci est incontournable pour rendre compte des pratiques de coordination. C'est le contexte de l'organisation qui sert de support à la pratique de coordination. Nous devons souligner que dans ce dernier cas, l'autorité du leader est complètement associée à son expertise qui est alors désignée comme mode de coordination. Nous ne pouvons pas avancer plus loin dans la comparaison car les situations extrêmes d'un centre traumatique relèvent de ce que nous avons appelé une situation d'urgence (Gautier, Lièvre, 2009) au même titre que les services secours incendie, dans le sens où ce ne sont pas les acteurs qui décident des problèmes à traiter alors que dans le cas d'une expédition polaire, ce sont bien les acteurs qui choisissent d'aller à tel endroit, même si dans le même temps, ils ne décident pas des problèmes qu'ils vont rencontrer en situation.

En se centrant délibérément sur les pratiques de " coordination en acte ", en ouvrant la boite noire de la coordination, les grammaires classiques de la coordination se brouillent et provoque la nécessité d'un dépassement conceptuel comme le soulignait déjà Alsene et Pichault (2007).

Ces premiers résultats sur les « pratiques de coordination en acte » en situation extrême pour un collectif informel amateur, sans standard de coordination préexistant, conduisent selon nous à s'interroger sur un certain nombre de notions selon deux axes : un approfondissement de ce que nous appelons un standard de coordination et un retour sur la nature de l'objet de la coordination.

Ne faut-il pas revenir sur cette question «lourde» de la définition de ce que nous appelons un standard de coordination ? A partir de quel moment peut-on parler d'un standard en matière de coordination ? Ne faut-il pas mieux préciser le caractère « formel » ou « informel » de ce standard ? Ne faut-il pas mieux spécifier les différentes formes de standard en fonction de la nature et de l'histoire de leur production (standard d'une procédure administrative issu du cerveau d'un énarque versus standard d'un processus de production issu d'un atelier auto-organisé 


\section{Pratiques de coordination d'un collectif informel en situation extrême : une étude de cas « ancrée » au Groenland}

ayant fait l'objet d'une procédure de norme de qualité) ? Ne faut-il pas mieux cerner le rapport qu'il établit entre la règle et l'action (quelques points de repères pour l'action versus une séquence précise d'opération permettant d'aboutir au but visé) ? Ne faut-il pas mieux identifier son niveau de reconnaissance social (local versus international), d'institutionnalisation (incorporé ou pas explicitement dans le schéma de fonctionnement de l'organisation, et aussi d'acquisition (sur le terrain versus dans le cadre d'une formation). Enfin ne faut-il pas mieux rendre compte de leur degré d'appropriation ou de réappropriation dans les pratiques elles-mêmes.

Ces questions sont déjà débattues et font l'objet de nombreux positionnements. Nizet et Pichault (2011) ont proposé dans leur article dans ce même numéro de donner des éléments de réponse à ces questions à partir d'une revue de littérature. Ainsi, ils sont amenés à concevoir le standard comme support à la coordination et à s'interroger sur les modalités d'interprétation que font les acteurs de ces standards en situation, en fonction des jeux de pouvoir en présence. Ce sont des avancées notables.

De notre coté, et dans ce prolongement et cette ouverture, nous interrogeons la question de l'investigation des pratiques de coordination du point de vue d'une épistémologie de la pratique. Si on s'inscrit résolument dans le cadre d'une épistémologie de la pratique, ne faut-il pas prendre comme point de départ les pratiques des acteurs au sens de disposition à agir non conscientisées en situation (schème, habitus), et non réductible au discours sur la pratique, et voir en quoi ces pratiques en situations relèvent effectivement de standards préexistants à la situation, et spécifier alors les caractéristiques de ces standards ? Ceci nous amènerait à suivre plusieurs pistes. Premièrement, la piste des invariants opératoires individuels (schème, habitus...) comme constitutifs des pratiques de coordination des acteurs. Quelles places prennent ces invariants opératoires individuels de nos conduites dans la construction de nos pratiques collectives ? II y a des liens à opérer entre différentes traditions psychologiques et sociologiques autour de ces questions : Piaget, Bourdieu, mais aussi Vigotsky... Deuxièmement, sur le plan de l'émergence de ces processus de coordination qui sont toujours a priori « singulier » et « irréversible », ne pourrions-nous pas mobiliser les travaux autour de la notion de routine organisationnelle développée par des auteurs comme Feldman et Pentland $(2003,2005)$ ? Ces travaux semblent pertinents tant par les éclairages apportés à nos débats, mais aussi par les propriétés qu'ils confèrent à ces routines qui font preuve autant de stabilité que d'adaptabilité.

Ne faut-il pas mieux définir la situation de gestion qui fait l'objet de la coordination? La synthèse proposée par Alsene et Pichaut que nous avons rappelé précédemment à partir des travaux de Fayol renvoie à ce qu'on pourrait appeler une coordination - organisation au sens d'organisation classique ayant à réaliser des opérations standards qui apparait en rupture avec la coordination-projet ou 
coordination-concourante (Declerck, Debourse et Navarre, 1983 ; Imai, Nonaka, Takeuchi, 1985) qui sont eux-mêmes en rupture avec la coordination-innovation au sens d'une organisation innovante-apprenante-créative (Nonaka, Takeuchi, 1995 ; Alter, 2004 ; Cohendet et alii, 2006). Comment spécifier ces différents registres définis dans la coordination-organisation pour leur donner du sens dans la coordination-innovation? Ne faut-il pas ajouter d'autres registres comme par exemple celui du retour d'expérience évoqué dans ce numéro par l'article de Cécile Godé (2011) mais aussi celui de l'apprentissage organisationnelle en situation qui renvoie à la question de la résilience organisationnelle développée par Karl Weick. Nous avons montré dans l'étude de cas que la coordinationorganisation permettait de rendre compte de la phase de préparation du projet, mais plus difficilement de la phase de réalisation.

Enfin pour terminer cet article, nous proposons de revenir un moment sur la question méthodologique. Nous avons indiqué en introduction les problèmes auxquels nous sommes confrontés lorsque nous voulons ouvrir la boite noire des pratiques de coordination. Cette difficulté méthodologique est évoquée dans le papier de Bouty et Drucker-Godard (2011) dans ce même numéro. La question n'est pas triviale. Nous avons rendu compte ici du dispositif que nous avons mis en œuvre tout au long du déroulement de ce projet. Ce dispositif ne va pas de soi : c'est un véritable construit en référence avec notre programme de recherche. Nous défendons la thèse qu'il est possible aujourd'hui de mobiliser un certain nombre d'outils méthodologiques propre à investir les pratiques des acteurs à distinguer du discours de rationalisation des acteurs sur leurs propres pratiques (Lièvre, Rix, 2009). C'est une investigation lourde mais qui semble à la hauteur de nos questionnements en matière de coordination dans le contexte d'une économie de l'innovation fondée sur la connaissance (Cohendet, Creplet, Dupouet, 2006).

\section{Bibliographie}

Alsène E., Pichault F. (2007), « La coordination au sein des organisations : éléments de recadrage conceptuel », Gérer et Comprendre, №87, p. 61-77.

Alter N. (2004), L'innovation ordinaire, PUF.

Bourdieu P. (1977), Le Sens Pratique. Editions de Minuit, Paris.

Bouty I., Drucker-Godard C. (2008), "Strategizing in high velocity environments: a routinized practice", 24th EGOS Annual Conference, Amsterdam, Netherlands.

Bouty I., Drucker-Godard C. (2011), "Emergence de l'agir collectif dans la course à la voile : rythme et coordination », Management \& Avenir.

Cohendet P., Creplet O., Dupouet O. (2006). Gestion des connaissances, firmes et communautés de savoir, Paris, Edition Economica, 205p.

Cook D.N. Brown J.S. (1999), « Bridging epistemologies: the generative dance between organizational knowledge and organizational knowing ", Organization Science, Vol 10, $\mathrm{N}^{\circ} 4$, p. 381-400.

Declerck R., Debourse D., Navarre C. (1983), Méthode de direction générale : le management stratégique de l'entreprise, Dunod. 


\section{Pratiques de coordination d'un collectif informel en situation extrême : une étude de cas « ancrée » au Groenland}

Faraj S., Xiao Y. (2006), "Coordination in fast-response organizations", Management Science, Vol. 52, N8, p. 1155-1169.

Feldman M.S., Pentland B.T. (2003), "Reconceptualizing organizational routines as a source of flexibility and change", Administrative Science Quarterly, 48: 94-118.

Foss N., Lorenzen M. (2009), "Towards an understanding of cognitive coordination: Theoretical development and empirical illustrations", Organization Studies, Vol. 30, №11, p. 1201-1226.

Gautier A. (2010), Modalités de mise en œuvre du retour d'expérience dans une perspective d'apprentissage organisationnel : le cas de l'organisation de la sécurité civile, Thèse de doctorat en sciences de gestion, Université Aix-Marseille II.

Girin J. (1990), «L'analyse empirique des situations de gestion : éléments de théorie et de méthode ", in A.-C. Martinet (dir.) : Epistémologies et sciences de gestion, Economica, Paris, p. 141-182.

Glaser B., Strauss A. (1967/2010), La découverte de la théorie ancrée, stratégies pour la recherche qualitative, Armand Colin.

Godé C. (2011), "Construire le sens par le retour d'expérience : le cas de l'équipe de voltige de l'armée de l'air », Management \& Avenir.

Godé-Sanchez C. (2010a), "Se coordonner en environnement volatil : les pratiques de coordination développées par les pilotes de chasse », Finance, Contrôle, Stratégie, Vol. $13, \mathrm{~N}^{\circ} 4$.

Godé-Sanchez C. (2010b),“Leveraging coordination in project-based activities: What can we learn from military teamwork?" Project Management Journal, Special Issue, 41(3), Project Management in Extreme Environnement.

Gupta P., Dirsmith M., Fogarty T. (1994), "Coordination and control in a government agency: contingency and institutional theory perspectives on GAO audits", Administrative Science Quarterly 39(2): 264-284.

Imaï K., Nonaka I., Takeuchi H. (1985), "Managing the new product development process : how japanese learn and unlearn", in The Uneasy Alliance: Managing the ProductivityTechnology Dilemma, dir. K. Clarck, R. Hayes et C. Lorenz, Boston, Harvard Business School Press.

Thompson J.D. (1967), Organizations in Action: Social Science Bases of Administrative Theory, Mc Graw-Hill, New York.

James W. (1907/2006), Le pragmatisme, Edition Flammarion.

James W. (1909/1998), La signification de la vérité, Edition Antipodes.

James W. (1912/2007), Essais d'empirisme radical, Edition Flammarion.

Keller, R. (1994), Technology-information processing fit and the performance of R\&D Project group: a test of contingency theory, Academy of Management Journal 37(1): 167179.

Lahire B. (2001), Le travail sociologique de Pierre Bourdieu, Edition La découverte.

Latour B. (2006), Changer de société - Refaire de la sociologie, Edition La Découverte.

Lave J., Wenger E. (1991), Situated Learning: Legitimate Peripheral Participation, NewYork, Cambridge University Press, 138p.

Lawrence, P.R., J.W. Lorsch (1989), Adapter les structures de l'entreprise: intégration ou différenciation, 1ère édition américaine en 1967, Paris, Éditions d'Organisation.

Lièvre P., Gautier A. (2009), "Les registres de la logistique des situations extrêmes : des expéditions polaires aux services d'incendie et de secours ", Management \& Avenir, Vol. 4, №24, p. 196-216.

Lièvre P., Rix-Lièvre G. (2009), « L'observatoire de l'organisant : mode d'interprétation des matériaux qui en sont issus ", Revue Internationale de Psychosociologie, Vol. 15, N35, p. 161-178.

Lièvre P., Rix-Lièvre G. (2009), « Vers l'émergence d'une nouvelle classe de méthodologie qualitative centrée sur le " faire ». Quelles perspectives pour la GRH ? » XX Colloque 
AGRH Toulouse.

Mintzberg H. (1982), Structure et dynamique des organisations, 1ère édition américaine en 1979, Paris/Montréal, Éditions d'Organisation/Agence d'Arc.

Nizet J., Pichault F. (2011), "Standards et jeux de pouvoir en situation extrême ", Management \& Avenir - ce numéro.

Paillé P. (2010), Introduction : Une enquête de théorisation ancrée, les racines et les innovations de la méthodologie de Glaser et Strauss, In B.G. Glaser, A. Strauss, « La découverte de la théorie ancrée ", Armand Colin.

Pentland B.T. et Feldman M.S. (2005), "Organizational routines as a unit of analysis", Industrial and Corporate Change, 14: 793-815.

Piaget J. (1936), La naissance de l'intelligence chez l'enfant, Delachaux et Niestlé.

Piaget J. (1974), La Prise de Conscience, Presses Universitaires de France, Paris.

Pichault F., Bouty I., Drucker-Godard C. Nizet J., Godé-Sanchez C., Lièvre P. (2010), "Coordination practices in extreme situations: Case studies, analytical framework and methodological stakes", $26^{\text {th }}$ EGOS Colloquium, July 2-4, Lisbon, Portugal.

Recopé M. (2001), « Milieux extrêmes et situation à risque : un point de vue constructiviste en référence à une théorie de l'action motrice ", in Pascal Lièvre, Logistique en milieux extrêmes, principes d'organisation des expéditions polaires à ski, Edition Hermès, p.181202.

Richardson R. (2006), "Abduction as the type of inference that characterizes the development about grounded theory", Qualitative Research, 6, 4, pp.497-513.

Ricoeur P. (1977), La sémantique de l'action, Edition du CNRS, Paris.

Rix G., Biache J.M. (2004) « Enregistrement en perspective subjective située et entretien en re situ subjectif : une méthodologie de constitution de l'expérience », Intellectica, ${ }^{\circ} 38$, p. 363-396.

Rix G., Lievre P. (2008), "Towards a codification of practical knowledge", Knowledge Management: Research and Practice, 6, 225-232.

Rix-Lièvre G. (2010), "Différents modes de confrontation à des traces de sa propre activité. Entre convergences et spécificités », Revue d'anthropologie des connaissances, 4 (2010/2), 357-376.

Rix-Lievre G., Lievre P. (2009), "Self-Confrontation Method", Encyclopedia Of Case Study Research, SAGE, Publication, dir. A. J. Mills, G. Durepos, E. Wieben.

Rix-Lièvre G., Lièvre P. (2010), "An innovative observatory of project of polar expeditions: the organizing's question", Project Management Journal, Special Issue, 41(3), Project Management in Extreme Environnement.

Schön D. A. (1983), The reflective practitioner, New York : Basic books,374p.

Van de Ven, A.H., Delbecq, A.L., Koenig, R. (1976), « Determinants of Coordination Modes within Organizations ", American Sociological Review, vol. 41, no. 2, pp. 322-338.

Vergnaud G. (1996), « Au cœur de l'action, la conceptualisation », in J.M. Barbier, Savoirs théoriques et savoirs d'action, Presses Universitaires de France.

Vergnaud G., Récopé M. (2000), « De Revault d'Allonnes à une théorie du schème aujourd'hui », Psychologie française, 45, 1, 35-50.

Weick K. (1993), "The collapse of sense-making in organizations: The Man Gulch disaster", Administrative Science Quarterly, Vol. 38, p.628-652.

Weick K. (1995), Sensemaking in Organizations, Sage Publications.

Yin R. (2003), Case Study Research: Design and Methods, Sage Publication, Applied Social Research Methods Series, Third Edition. 


\title{
La résolution des problèmes concrets de coordination en situation extrême : essai de synthèse
}

\author{
par Isabelle Bouty ${ }^{255}$, Carole Drucker-Godard ${ }^{256}$, \\ Cécile Godé ${ }^{257}$, Pascal Lièvre ${ }^{258}$, \\ Jean Nizet ${ }^{259}$ et François Pichault ${ }^{260}$
}

Les quatre analyses que nous avons menées, qui développent chacune une problématique spécifique et reposent sur une base empirique globale de cinq cas diversifiés, nous permettent de tenter une réponse à la question qui motive ce cahier spécial : comment les acteurs résolvent-ils concrètement les problèmes de coordination en situation extrême? Rappelons qu'une situation extrême est une situation de gestion caractérisée par l'évolutivité (décalages rapides par rapport à des modes de fonctionnement antérieurs ou habituels), l'incertitude (faible probabilité d'occurrence et/ou difficulté, voire impossibilité, d'anticipation) et le risque (possibilité de dommages à l'organisation et ou à ses acteurs).

Nos analyses suggèrent que les pratiques de coordination déployées par les acteurs dans ce type de contexte s'articulent autour de quatre composants de base: $1^{\circ}$ elles s'appuient sur des standards; $2^{\circ}$ elles s'accompagnent d'un travail de construction collective de sens; $3^{\circ}$ des choix sont opérés en permanence sur la distribution des rôles et activités entre acteurs et posent la question du pouvoir et du leadership; $4^{\circ}$ ces pratiques s'inscrivent dans des temporalités structurées.

Contrairement aux thèses de la littérature contingente (parmi d'autres : Galbraith, 1973; Mintzberg, 1982), mais en accord avec un ensemble de recherches plus récentes (par exemple, Bigley et Roberts, 2001; Faraj et Xiao, 2006), nos analyses montrent toute l'importance des standards dans la coordination en situation extrême. Ces standards varient suivant leurs modalités: certains s'incarnent dans des objets ou dispositifs matériels (la vidéo des vols dans le cas de la voltige aérienne); d'autres se présentent sous la forme d'écrits (les listes de procédures dans le cas des frappes aériennes en Afghanistan ou les protocoles de lecture

\footnotetext{
255. Isabelle Bouty, Maître de Conférences, Ceros, Université Paris Ouest Nanterre la Défense, isabelle.bouty@u-paris10.fr 256. Carole Drucker-Godard, Maître de Conférences, Ceros, Université Paris Ouest Nanterre la Défense, carole.godard@u-paris10.fr

257. CÉCILE GodÉ, Chercheur au Centre de Recherche de l'Armée de l'Air (CReA), GREDEG UMR 6227 CNRS, Université de Nice Sophia-Antipolis, cecile.gode@inet.air.defense.gouv.fr

258. PAsCAL LiėVRE, Maître de Conférences HDR, Clermont Université \& Groupe ESC Clermont, CRCGM EA 3849

pascallievre@orange.fr

259. Jean Nizet, Professeur extraordinaire aux Facultés Universitaires Notre Dame de la Paix à Namur et Professeur à l'Université Catholique de Louvain, jean.nizet@fundp.ac.be

260. François Pichault, HEC-Ecole de gestion de I'Université de Liège, f.pichault@ulg.ac.be
} 
des clichés échographiques dans le cas du suivi de grossesse à risques). Ils varient aussi suivant leur origine: à côté de ceux qui sont mis au point par les analystes de l'organisation (les procédures formalisées de retour d'expérience des pilotes de chasse, évoquées par Godé), certains trouvent leur origine dans les communautés professionnelles auxquelles appartiennent les acteurs concernés (les guidelines issus des recherches médicales), tandis que d'autres encore renvoient à un cadre réglementaire émanant des institutions dont dépend l'organisation étudiée (les règles émises par les gouvernements des pays dont les armées interviennent en Afghanistan). Certes, tous ne structurent pas de la même manière les pratiques de coordination. Dans certaines circonstances, les effets semblent directs, immédiats (les programmes imposés lors des compétitions de voltige), limitant étroitement les possibilités de travail interprétatif de la part des opérateurs tandis que dans d'autres, l'influence apparaît plus médiate: elle passe par un important travail interprétatif effectué par les acteurs (le cas des médecins du service de néonatologie), ce qui nous renvoie au deuxième composant que nous aborderons plus bas.

L'importance des standards est illustrée, a contrario, par le cas de l'expédition polaire au Groenland analysée par Lièvre et Rix-Lièvre. II apparaît de fait que l'absence de standard organisationnel préexistant et partagé entre les membres de ce collectif informel pose problème. Tout se passe donc comme s'il fallait, en situation, pallier une telle absence, à savoir construire ex nihilo des routines organisationnelles. Parmi les cinq cas étudiés, c'est assurément celui qui manifeste le plus clairement des défauts de coordination, par exemple dans le mode de progression quotidien de l'expédition sur le terrain (démarrage, pause et arrêt pour le camp), l'épisode de la perte des chiens, ou encore dans la prise de décision de l'abandon du sommet. On n'observe ici ni la présence de standards institutionnels, ni celle de standards organisationnels. Les connaissances et compétences du guide, consignées dans l'ouvrage qu'il a publié, auraient certes pu faire office de standards professionnels, mais elles ne sont pas connues par les autres participants, ne permettent pas de répondre aux problèmes soulevés (elles suscitent des réserves de la part de ces autres acteurs) et ne contribuent donc guère à la coordination des tâches dans cette situation.

Les standards donnent lieu, de la part des acteurs, à une construction collective de sens; celle-ci constitue le deuxième composant de la coordination en situation extrême. De nombreux travaux ont indirectement suggéré, en opposant communication directe et standardisation comme modes de coordination (Van de Ven et al., 1976 ; Argote, 1982; Keller, 1994 ; Malone et Crowston, 1994 ; Crowston, 1997 ; Gittell, 2002 par exemple) que la nécessité d'une construction collective de sens apparaissait de facto lorsque les arrangements prédéfinis étaient inopérants. Mais au fil des différentes études qui composent ce dernier dossier, nous montrons que tel n'est pas le cas, bien au contraire : loin d'être opposables l'un à l'autre, standards et construction collective de sens sont 


\section{La résolution des problèmes concrets de coordination en situation extrême : essai de synthèse}

complémentaires. En effet, dans le cas de la voile, c'est parce que les membres de l'équipe se sont accordés sur une représentation commune de la situation à un moment donné qu'ils peuvent mobiliser les standards de façon pertinente (le fameux " maintenant »). L'exercice de construction collective de sens est en quelque sorte un préalable nécessaire au recours aux standards, non un substitut. Le cas de l'équipe de voltige de l'Armée de l'air illustre également cette complémentarité entre construction collective de sens et standards. Cependant, à la différence de la voile, c'est l'existence préalable du langage standardisé (le code Aresti) qui nourrit le processus d'élaboration collective de sens, et non l'inverse. Les standards facilitent la communication entre les membres, qui s'y réfèrent pour construire leurs actions et gérer leurs interactions. Ils favorisent ainsi une compréhension mutuelle des activités de chacun au sein du groupe et alimentent la dimension identitaire et sociale de celui-ci. Dans le cas du service de néonatologie, la construction de sens prend encore une forme différente: les standards professionnels (les guidelines auxquels se réfèrent les médecins) contribuent moins à doter les acteurs de représentations communes qu'à leur offrir une toile de fond susceptible de donner sens à leurs débats et négociations. Et dans le cas de l'expédition au Groenland, la quasi-absence des standards et la tentative d'imposition de sens par l'un des protagonistes privent les acteurs de références communes, chacun se comportant dès lors en fonction de ses perceptions et de ses choix propres.

La complémentarité que nous avons observée entre construction collective de sens et standards converge avec les phénomènes décrits par des auteurs tels que Bigley et Roberts (2001) ou Weick (1993) qui ont mis en évidence la complémentarité entre structuration des activités (y compris sous un mode bureaucratique) et communication interpersonnelle dans la construction de représentations opérationnelles partagées. On retrouve aussi les travaux de certains auteurs en gestion de projet (Yakura, 2002 ; Sapsed et Salter, 2004) selon lesquels les techniques de planification jouent en fait un rôle d'objets-frontières, dotant les différents protagonistes d'un référentiel commun de connaissances en vue de mener à bien leurs négociations. Nos travaux montrent qu'une telle complémentarité peut être étendue aux standards et constitue dès lors un des traits spécifiques de la coordination en situation extrême.

Les phénomènes de pouvoir et de leadership constituent le troisième composant de ces pratiques de coordination. En effet, le travail interprétatif à la base de la construction collective de sens est fortement marqué par la nature des relations de pouvoir entre acteurs. Plus celles-ci sont symétriques (comme dans le cas du suivi de grossesse à risque), plus le travail interprétatif de la situation extrême sera intense et donnera lieu à de multiples échanges entre acteurs; plus elles sont asymétriques (comme dans les cas des compétitions de voltige ou de voile), plus le travail interprétatif sera cadenassé et limité à des circonstances exceptionnelles. Toutefois, la nécessité de s'adapter en permanence à des situations imprévisibles 
amène fréquemment à des redistributions des rôles et des activités entre acteurs qui peuvent « redistribuer les cartes » et poser de redoutables problèmes de leadership. Le cas le plus flagrant est celui de l'expédition au Groenland, relatée par Lièvre et Rix-Lièvre. En l'absence de standards communs et de représentations partagées de la situation, on assiste à une remise en cause du leadership du chef d'expédition sur la question de la sécurité ours : c'est Gilles et Pierre qui sont affectés intégralement à cette mission, mais Pierre et Cloé, par moments, sont en charge du suivi des chiens en progression, et c'est enfin Pierre qui bricole une solution au départ des chiens. Pierre prend de facto le rôle du chef d'expédition, quand ce dernier est en difficulté, par exemple lors de son passage à l'eau sur la rivière glacée. C'est également Pierre qui va prendre en main la conduite de l'expédition jusqu'à ce que Joël soit " sauf » et « sec ». Ces différentes situations fragilisent la légitimité du chef d'expédition et mettent en évidence le fait que si aucun acteur n'est en charge de la formalisation des pratiques de coordination, le risque est grand de se trouver devant un exercice plus personnel, voire intuitif du pouvoir par certains acteurs : l'efficacité d'une telle pratique de coordination dépend, en définitive, des relations que ces acteurs sont capables de nouer, en situation, avec les autres acteurs impliqués. On est ainsi renvoyé à la vulnérabilité du recours aux relations interpersonnelles comme mécanisme de coordination, qui repose tout entier sur la confiance préalable entre acteurs ; lorsque celleci n'est pas présente, le risque d'opportunisme de la part de certains acteurs est élevé (Bornarel, 2007) et vient s'ajouter au caractère risqué de la situation extrême elle-même. Dans ces conditions, il n'est pas étonnant de voir apparaître des comportements de retrait (exit) ou de contestation (voice) (Hirschman, 1970). Dans le cas de l'expédition polaire, le chef d'expédition a bien tenté, en amont, de créer les conditions de la confiance en veillant avec soin à la composition de l'équipe : mais ces précautions n'ont pas suffi pour faire face aux turbulences de la situation extrême, en l'absence de tout standard partagé par les membres de l'équipe.

II va de soi que la redistribution des rôles et des activités entre acteurs s'opère également en présence de standards, mais sans nécessairement mettre en cause la légitimité du leader. Ainsi, dans le cas de la coordination air/sol au cours des opérations militaires en Afghanistan, la distribution des rôles pourtant extrêmement formalisée entre le pilote, le navigateur et le contrôleur peut évoluer pour faire face à des situations tactiques imprévues, conduisant à s'écarter du plan prévu initialement. II leur arrive alors de décider du mode d'intervention en retournant au langage naturel. Mais cette décision fera l'objet d'un debriefing formel puis informel, et d'une validation éventuelle par la hiérarchie. Dans le cas de l'équipe du tour de France à la voile, relaté par Bouty et Godard, les rôles sont fortement distribués puisqu'un membre d'équipage ne peut occuper qu'un seul poste sur le bateau. Par contre, la distribution des acteurs peut être différente en fonction des régates ; même le skipper peut changer. Ceci est rendu possible, car tous les acteurs peuvent quasiment occuper tous les postes sur le bateau; de 


\section{La résolution des problèmes concrets de coordination en situation extrême : essai de synthèse}

par leurs expériences, ils sont tous polyvalents. Mais comme le montre l'étude de cas, une fois l'équipage constitué, l'essentiel de la communication repose sur trois acteurs: le barreur, le technicien et le tacticien qui vont de fait conduire la coordination sur le bateau et plus particulièrement son rythme. Les changements opérés dans la distribution des rôles n'affectent en rien la légitimité de ceux-ci. Enfin, dans le cas du suivi de grossesse à risque, le collectif qui suit le dossier est mouvant, voire aléatoire, ce qui oblige d'ailleurs à documenter les décisions prises en référence au patient, mais une fois encore, cette évolution dans la prise en charge ne remet guère en question la légitimité des intervenants. On voit donc combien la redistribution des rôles et des activités en situation extrême peut affecter différemment la légitimité des leaders et les relations de pouvoir entre acteurs selon qu'elle s'appuie, ou non, sur l'existence de standards.

Nos analyses montrent abondamment que ces trois composants ne jouent pas isolément dans les pratiques de coordination, mais qu'ils interagissent constamment, autrement dit, qu'ils forment un système. La comparaison des deux cas traités par Nizet et Pichault (les frappes en Afghanistan et le suivi de la grossesse à risque) fait bien apparaître en quoi la configuration du pouvoir dans l'organisation interagit avec le travail de construction de sens et avec la manière dont les standards influencent les pratiques. Et dans le cas de l'expédition au Groenland étudiée par Lièvre et Rix-Lièvre, la quasi-absence de standards et de construction commune de sens n'est pas sans rapport avec le mode de leadership adopté par l'initiateur du projet, ni d'ailleurs avec tout le soin qu'il a mis à recruter les membres de l'expédition, comme s'il cherchait à assurer, par une sélection a priori, un sens partagé que la pratique de la coordination ne pouvait pas assurer en cours de route.

On soulignera enfin que les trois premiers composants caractéristiques de la coordination du travail en situation extrême s'inscrivent dans diverses formes de temporalités. Ce quatrième composant ressort fortement de nos analyses même si, suivant les thématiques abordées et/ou les cas analysés, les temporalités en question sont très différentes. On peut les ranger depuis le long terme jusqu'au court terme.

- Prend place dans le plus long terme - quelques semaines, quelques mois, voire quelques années - l'évolution des standards institutionnels tels que ceux identifiés dans le cas des forces aériennes engagées en Afghanistan : il faut en effet plusieurs années pour que les réglementations fixées par les gouvernements évoluent à la suite des évaluations des actions de terrain ; mais aussi celle des standards professionnels dans le cas du suivi de la grossesse à risque (plusieurs années également pour consigner les observations, rédiger et publier les recherches scientifiques). On situera également dans le long terme la formation des acteurs concernés (par exemple dans le cas des frappes en Afghanistan), qui leur permet de maîtriser les instructions, 
d'utiliser les langages spécifiques, mais aussi d'acquérir des représentations communes de la situation, ainsi que d'assurer la cohésion du groupe.

- On observe, à l'autre extrême, des structurations du temps sur le très court terme (quelques minutes). On rappellera notamment le " rythme » qui caractérise les pratiques de coordination dans les courses à la voile, telles que Bouty et Godard les ont décrites. Dans le cas de l'expédition au Groenland, l'organisation du sauvetage de l'équipier qui tombe à l'eau est une situation imprévue qui demande une réaction d'une grande rapidité de la part du reste de l'équipe : en trois minutes, une corde est lancée... et dix minutes après, l'équipier est au sec.

- Enfin, la structuration du temps prend parfois des formes intermédiaires (plusieurs heures, plusieurs jours). Une illustration simple en est le « colloque du lundi » du service de néonatologie, où les professionnels débattent de l'état des patientes et confrontent leurs options quant au suivi médical et humain à assurer. Godé nous offre un exemple plus complexe, quand elle montre comment, dans le cas de la voltige, les différentes « boucles d'apprentissage » s'enchâssent et s'alimentent l'une l'autre: le retour d'expérience en temps réel, pendant le vol; les débriefings vidéo et les discussions informelles, tout de suite après; enfin, les échanges en salle de repos, plusieurs heures plus tard.

Ces différentes temporalités, dont nous venons de donner des exemples isolés, se chevauchent, et dans certaines circonstances s'emboîtent, constituant ainsi, pour chaque situation, une structure temporelle qui peut être relativement complexe. Reprenons le cas de l'expédition au Groenland. II y a d'abord le temps long de la conception, qui peut s'étaler sur plusieurs années : c'est alors que se déploient l'exploration et l'ingéniosité du chef d'expédition. II y a aussi le temps long de la préparation collective (de l'ordre de l'année), avec une véritable course contre la montre qui s'enclenche à partir du moment où la date du départ est fixée. Puis, il y a le temps intermédiaire de l'expédition sur le terrain, qui se compte en jours ou en semaines. Pendant cette phase de réalisation, il y a un emboîtement de deux temporalités : d'une part, celle du déroulement quotidien de l'expédition avec tous ses impondérables et, d'autre part, celle de la traversée proprement dite, avec un point fixe à l'arrivée comme date butoir. Dans le déroulement quotidien, il s'agit de construire progressivement des routines collectives selon un rythme extrêmement régulier: lever, démontage du camp, progression, pause, progression, montage du camp. Dans un contexte d'incertitude, où il faut trouver le bon itinéraire sur la banquise, sur des rivières gelées, il faut assurer le déplacement quotidien quels que soient les problèmes météorologiques et les difficultés physiques et techniques des uns et des autres. Comme nous l'avons vu, le moindre incident - comme la chute à l'eau d'un équipier - peut bloquer la caravane pendant plusieurs jours... Face à cette temporalité quotidienne, continuellement ajustée, il y a la temporalité de la traversée qui oblige à se retrouver au point d'arrivée 


\section{La résolution des problèmes concrets de coordination en situation extrême : essai de synthèse}

à un moment donné. Les possibilités de liaisons aériennes sont limitées et les réserves en nourriture de l'équipe sont calibrées ${ }^{261}$.

C'est en particulier la capacité à articuler ces diverses temporalités qui caractérise la coordination des activités en situation extrême: les temps courts liés à l'imprévisibilité des événements ne peuvent " faire sens " pour les acteurs qu'en se greffant sur les temps intermédiaires et longs durant lesquels se constitue le socle de leurs représentations communes. En d'autres termes, le temps passé à la formation, à la conception, à la préparation des activités en situation extrême est en quelque sorte un investissement permettant aux rythmes courts de la coordination de se développer de manière signifiante aux yeux des protagonistes.

En synthèse, la résolution des problèmes concrets de coordination en situation extrême fait donc appel, contrairement à ce qu'une vision trop mécanique de la coordination pourrait donner à penser, à des standards de forme et d'origine variée. Ces standards peuvent s'avérer plus ou moins ouverts au travail interprétatif des acteurs selon qu'ils émanent d'autorités politiques ou réglementaires (standards institutionnels), d'acteurs en position d'analystes (standards organisationnels) ou de la communauté à laquelle appartiennent les acteurs concernés (standards professionnels). Ces standards sont mobilisés corrélativement à un travail de construction collective de sens, plus ou moins intense, qui repose largement sur la légitimité d'un leader et/ou sur la nature des relations (de coopération, d'opposition, voire de retrait) existant entre acteurs. Nous avons souligné que toute redistribution des rôles et des activités, en fonction de l'évolution concrète de la situation, risque de conduire à une remise en cause de la légitimité du leader, particulièrement en l'absence de standards. Enfin, l'investissement consenti dans les temporalités longues (en formation, préparation, etc.) garantit la plus ou moins grande efficacité, en situation, du recours aux temporalités courtes. L'ensemble de ces composants est en étroite interrelation et forme ce qu'on peut appeler un système évolutif de coordination, comme le montre le schéma suivant.

\footnotetext{
261. On notera que ces diverses temporalités ont également un ancrage spatial, à tel point que l'on peut se risquer à parler de structures spatio-temporelles. Si l'on reprend le cas de l'expédition polaire, sur le temps long, il y a la préparation qui s'effectue en France, le déplacement au Groenland, le retour en France, etc. Sur le temps intermédiaire, la traversée est indissociablement faite de temps qui passe mais aussi d'espaces parcourus, etc. Dans le cas des frappes aériennes en Afghanistan, il y a le décollage et le retour à la base dans le temps intermédiaire, l'alternance sol/l'air dans le temps court, etc.
} 
Le système évolutif de coordination des activités en situation extrême

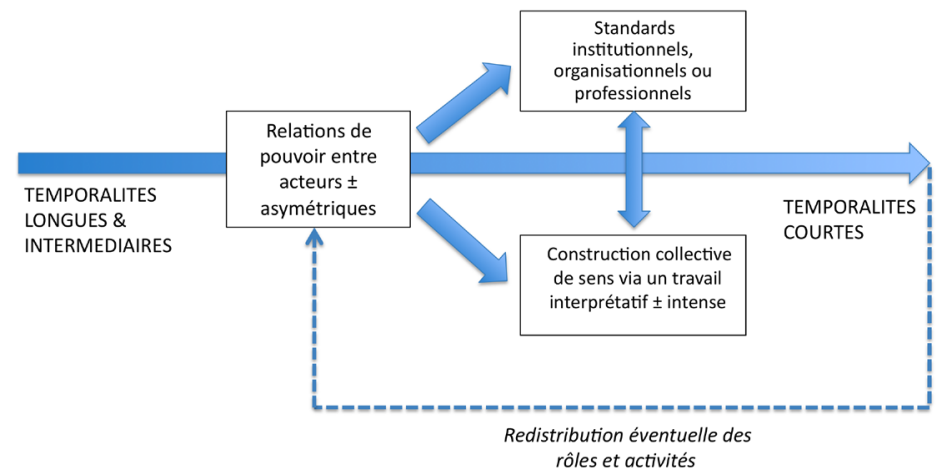

\section{Références}

Argote, L. (1982), " Input Uncertainty and Organizational Coordination in Hospital Emergency Units », Administrative Science Quarterly, vol.27, n³, pp.420-434.

Bigley, G., \& Roberts, K. (2001), "The Incident Command System: High Reliability Organizing for Complex and Volatile Task Environments », Academy of Management Journal, vol.44, n6, pp.1281-1299.

Bornarel, F. (2007), « La confiance comme instrument d'analyse de l'organisation », Revue française de gestion, $\mathrm{n}^{\circ} 175$, pp.95-109.

Crowston, K. (1997), "A Coordination Theory Approach to Organizational Process Design", Organization Science, Vol. 8, N², p. 157-175.

Faraj, S. \& Xiao, Y. (2006), " Coordination in Fast-Response Organizations », Management Science, vol. $52, n^{\circ} 8$, pp.1155-1169.

Galbraith, J.R. (1973), Designing Complex Organizations, Addison-Wesley, Reading.

Gittell, J. (2002), "Coordinating Mechanisms in Care Providing Groups: Relational Coordination as a Mediator and Input Uncertainty as a Moderator of Performance Effects", Management Science, Vol. 48, №11, p. 1408-1426.

Hirschman, A.O. (1970), Exit, Voice, and Loyalty: Responses to Decline in Firms, Organizations, and States, Cambridge (Massachusetts), Harvard University Press.

Keller, P. (1994), "Technology-Information Processing Fit and the Performance of R\&D Project Group: a Test of Contingency Theory", Academy of Management Journal, Vol. 37, $\mathrm{N}^{\circ} 1$, p. 167-179.

Lawrence, P.R. \& J.W. Lorsch (1989), Adapter les structures de l'entreprise: intégration ou différenciation, 1ère édition américaine en 1967, Paris, Éditions d'Organisation.

Malone, T.W. \& Crowston, K. (1994), « The Interdisciplinary Study of Coordination », ACM Computing Surveys, vol. 26 (1), pp. 87-119.

Mintzberg, H. (1982), Structure et dynamique des organisations, 1ère édition américaine en 1979, Paris/Montréal, Éditions d'Organisation/Agence d'Arc.

Sapsed, J. \& Salter, A. (2004) « Postcards from the Edge: Local Communities, Global Programs and Boundary Objects », Organization Studies, vol.25, n9, pp.1515-1534.

Yakura, E. K. (2002) « Charting Time: Timelines as Temporal Boundary Objects », Academy of Management Journal, vol.45, n5, pp.956-970.

Van de Ven, A.H., Delbecq, A.L. \& Koenig, R. (1976), " Determinants of Coordination Modes within Organizations ", American Sociological Review, vol. 41, no. 2, pp. 322338.

Weick, K. E., "The Collapse of Sensemaking in Organizations: The Mann Gulch Disaster", Administrative Science Quarterly, Vol. 38, N4, p. 628-652. 